\title{
Non-classical problems for viscoelastic solids with microstructure
}
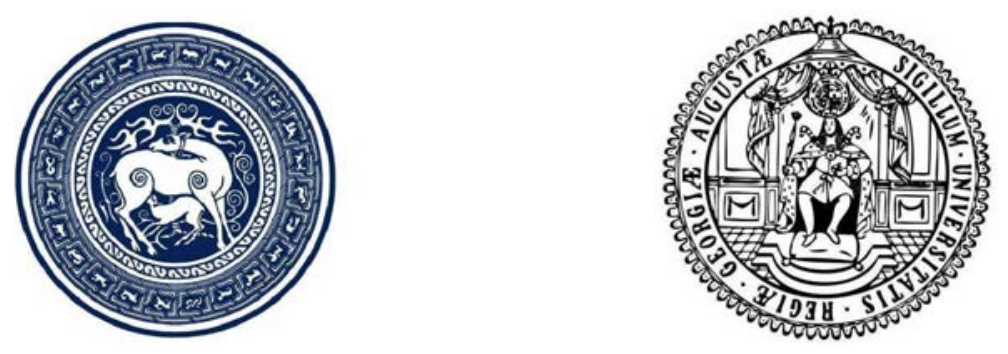

Maia M. Svanadze

I. Javakhishvili Tbilisi

State University
Georg-August-Universität

Göttingen

A thesis submitted for the degree of

PhD in Mathematics

Doctor of Philosophy 


\section{Acknowledgements}

I am enormously grateful to my supervisors Professor George Jaiani (I. Javakhishvili Tbilisi State University) and Professor Ingo Witt (University of Göttingen) for their invaluable guidance and encouragement.

In addition, I would like to thank Shota Rustaveli National Sciences Foundation (Georgia) for PhD student grant and The University of Göttingen (Germany) for two years grant scholarship.

Maia M. Svanadze

Tbilisi, Göttingen

May, 2014 


\begin{abstract}
In the present thesis the linear theories of viscoelasticity and thermoviscoelasticity for isotropic and homogeneous Kelvin-Voigt materials with voids are considered and some basic results of the classical theories of elasticity and thermoelasticity are generalized. Indeed, the basic properties of plane harmonic waves in the linear theory of viscoelasticity for Kelvin-Voigt materials with voids are established. There are two longitudinal and two transverse attenuated plane waves in the Kelvin-Voigt material with voids. In the considered theories the fundamental solutions of the systems of equations of steady vibrations are constructed by means of elementary functions and their basic properties are established. The representations of Galerkin type solutions of the systems of equations of steady vibrations are obtained. The Green's formulas and integral representations of Somigliana type of regular vector and classical solutions are obtained. The formulas of representations of the general solution for the system of homogeneous equations of steady vibrations are established. The completeness of these representations of solutions is proved. The uniqueness theorems of the internal and external boundary value problems (BVPs) of steady vibrations in the linear theories of viscoelasticity and thermoviscoelasticity for Kelvin-Voigt materials with voids are proved. The basic properties of surface (single-layer and double-layer) and volume potentials are studied. On the basis of these potentials the BVPs are reduced to the singular integral equations. The corresponding singular integral operators are of the normal type with an index equal to zero. The Fredholm's theorems are valid for these singular integral operators. Finally, the existence theorems of classical solutions of the above mentioned BVPs of the linear theories of viscoelasticity and
\end{abstract}


thermoviscoelasticity for Kelvin-Voigt materials with voids are proved by using the potential method (boundary integral equation method) and the theory of singular integral equations. 


\section{Contents}

Contents

1 Introduction 1

1.1 Thesis structure . . . . . . . . . . . . . . . . . 1

1.2 On the theories of viscoelasticity and thermoviscoelasticity: Literature review . . . . . . . . . . . . . . . . 3

1.3 Basic notations . . . . . . . . . . . . . . . . . 7

2 Solutions of equations in the theory of viscoelasticity for mate$\begin{array}{ll}\text { rials with voids } & 10\end{array}$

2.1 Basic equations . . . . . . . . . . . . . . . . . . . . 10

2.2 Solution of the dispersion equations. Plane harmonic waves . . . . 13

2.3 Fundamental solution . . . . . . . . . . . . . . . . . . . . . 17

2.4 Green's formulas. Representations of general solutions . . . . . . . 22

3 Boundary value problems in the theory of viscoelasticity for ma$\begin{array}{ll}\text { terials with voids } & 30\end{array}$

3.1 Basic boundary value problems . . . . . . . . . . . . . . 30

3.2 Uniqueness theorems . . . . . . . . . . . . . . . . . . . 31

3.3 Basic properties of elastopotentials . . . . . . . . . . 36

3.4 Existence theorems . . . . . . . . . . . . . . . . . . . . . . . . 41

4 Solutions of equations in the theory of thermoviscoelasticity for materials with voids $\quad 46$

4.1 Basic Equations . . . . . . . . . . . . . . . . . . . 46 
4.2 Fundamental solution . . . . . . . . . . . . . . . . . . 50

4.3 Galerkin type solution . . . . . . . . . . . . . 56

4.4 Representation of general solution of system of homogeneous equations . . . . . . . . . . . . . . . . . . 59

4.5 Green's formulas. Integral representation of solution . . . . . . . 66

5 Boundary value problems in the theory of thermoviscoelasticity for materials with voids $\quad 72$

5.1 Basic boundary value problems . . . . . . . . . . . 72

5.2 Uniqueness theorems . . . . . . . . . . . . . . . 74

5.3 Basic properties of thermoelastopotentials . . . . . . . . . 81

5.4 Existence theorems . . . . . . . . . . . . . . . . . . 85

6 Concluding remarks $\quad 91$

$\begin{array}{lr}\text { References } & 94\end{array}$ 


\section{Chapter 1}

\section{Introduction}

\subsection{Thesis structure}

The structure of the thesis is as follows: the content of this thesis is divided into six chapters. Chapters 2 to 5 can be roughly divided into two parts. The first part (Chapters 2 and 3) and the second part (Chapters 4 and 5) include the investigation of problems of the linear theories of viscoelasticity and thermoviscoelasticity for Kelvin-Voigt materials with voids, respectively. In the final chapter the basis results of this thesis are summarized and some fields of application of these results are analyzed.

Each Chapter is articulated as follows:

In the next sections of this chapter (Sections 1.2 and 1.3) a review of the literature on the theories of viscoelasticity and thermoviscoelasticity is presented and the basic notations are given. These notations are used throughout this thesis.

Chapter 2 (Sections 2.1 to 2.4) is focused on the solutions of the system of equations of steady vibrations in the linear theory of viscoelasticity for isotropic and homogeneous Kelvin-Voigt materials with voids. Indeed, the governing equations of steady vibrations of the linear theory of viscoelasticity are given. The basic properties of solutions of the dispersion equations of longitudinal and transverse plane harmonic waves are studied. The fundamental solution of the system of equations of steady vibrations is constructed by means of elementary func- 
tions and its some basic properties are established. Finally, Green's formulas and integral representations of general solutions of the above mentioned system of equations are obtained.

In Chapter 3 (Sections 3.1 to 3.4) the basic internal and external BVPs of steady vibrations of the linear theory of viscoelasticity for Kelvin-Voigt materials with voids are investigated using the potential method and the theory of singular integral equations. Indeed, the basic BVPs are formulated and the uniqueness theorems of classical solutions of these BVPs are proved. The basic properties of the elastopotentials and the singular integral operators are established. On the basis of these potentials the BVPs are reduced to the singular integral equations. The corresponding singular integral operators are of the normal type with an index equal to zero. The Fredholm's theorems are valid for these singular integral operators. Finally, the existence theorems of classical solutions of the BVPs of steady vibrations are proved.

Chapter 4 (Sections 4.1 to 4.5 ) treats the solutions of the system of equations of steady vibrations in the the linear theory of thermoviscoelasticity for KelvinVoigt materials with voids. Indeed, the governing equations of steady vibrations of the linear theory of thermoviscoelasticity are given. The fundamental solution of the system of equations of steady vibrations is constructed by means of elementary functions and its some basic properties are established. The Galerkin type solution of the system of nonhomogeneous equations and the representation of general solution of the system of homogeneous equations are obtained. The Green's formulas and integral representations of general solutions of the systems of equations of steady vibrations are presented.

In Chapter 5 (Sections 5.1 to 5.4) the basic internal and external BVPs of steady vibrations of the linear theory of thermoviscoelasticity for Kelvin-Voigt materials with voids are investigated using the potential method and the theory of singular integral equations. Indeed, the basic BVPs are formulated and the uniqueness theorems of classical solutions of these BVPs are proved. The basic properties of the thermoelastopotentials (single-layer, double-layer and volume potentials) and the singular integral operators are established. On the basis of these potentials the BVPs are reduced to the singular integral equations. The corresponding singular integral operators are of the normal type with an index 
equal to zero. Therefore, Fredholm's theorems are valid for these singular integral operators. Finally, the existence theorems of classical solutions of the BVPs of steady vibrations are proved.

The main results of the Chapters 2 to 5 are published in the papers of author of this thesis (see Svanadze $[1-4]$ ).

\subsection{On the theories of viscoelasticity and ther- moviscoelasticity: Literature review}

The theories of viscoelasticity initiated by J. C. Maxwell, O. E. Meyer, L. Boltzmann, and studied by W. Voigt, Lord Kelvin (W. Thomson), S. Zaremba, V. Volterra and others. These theories, which include the Maxwell model, the Kelvin-Voigt model, and the standard linear solid model, were used to predict a material's response under different loading conditions (see Eringen [5], Truesdell and Noll [6], Christensen [7], Amendola et al. [8]).

Viscoelastic materials play an important role in many branches of civil engineering, geotechnical engineering, technology and, in recent years, biomechanics. Viscoelastic materials, such as amorphous polymers, semicrystalline polymers, and biopolymers, can be modeled in order to determine their stress or strain interactions as well as their temporal dependencies (see Shaw and MacKnight [9], Ferry [10]). Study of bone viscoelasticity is best placed in the context of strain levels and frequency components associated with normal activities and with applications of diagnostic tools (see Lakes [11]). The investigations of the solutions of viscoelastic wave equations and the attenuation of seismic wave in the viscoelastic media are very important for geophysical prospecting technology. In addition, the behavior of viscoelastic porous materials can be understood and predicted in great detail using nano-mechanics. The applications of these materials are many. One of the applications may be to the NASA space program, such as the prediction of soils behavior in the Moon and Mars (for details, see Voyiadjis and Song [12], Polarz and Smarsly [13], Chen et al. [14], Gutierrez-Lemini [15] and references therein).

A great attention has been paid to the theories taking into account the vis- 


\subsection{On the theories of viscoelasticity and thermoviscoelasticity: Literature review}

coelastic effects (see Amendola et al. [8], Fabrizio and Morro [16], Di Paola and Zingales $[17,18])$. The existence and the asymptotic stability of solutions in the linear theory of viscoelasticity for solids are investigated by Fabrizio and Lazzari [19], and Appleby et al. [20]. The main results on the free energy in the linear viscoelasticity are obtained in the series of papers [21 - 28]. A general way to provide existence of the initial and BVPs for linear viscoelastic bodies is provided without the need of appealing to transient solutions is presented by Fabrizio and Morro [16], Fabrizio and Lazzari [19], and Deseri et al. [21].

Material having small distributed voids may be called porous material or material with voids. The intended application of the theory of elastic material with voids may be found in geological materials like rocks and soils, in biological and manufactured porous materials for which the theory of elasticity is inadequate. But seismology represents only one of the many fields where the theories of elasticity and viscoelasticity of materials with voids is applied. Medicine, various branches of biology, the oil exploration industry and nanotechnology are other important fields of application.

The theories of elasticity and thermoelasticity for materials with voids have been a subject of intensive study in recent years. The initial variant (linear and non-linear) of the theory of elasticity for materials with voids proposed by Nunziato and Cowin $[29,30]$ and developed by several authors in the series of papers [31 - 40]. A linear theory of thermoelastic materials with voids was considered and the acceleration waves were studied by Ieşan [41]. Scalia [42] considered a grade consistent micropolar theory of thermoelasticity for materials with voids. The Galerkin type solution in the theory of thermoelastic materials with voids was constructed by Ciarletta [43]. The steady vibrations problems of this theory was investigated by Pompei and Scalia [44]. The spatial and temporal behavior of solutions in linear thermoelasticity of solids with voids were studied by Chiriţă and Scalia [45]. The basis properties of the acceleration and plane harmonic waves in this theory were established by Ciarletta and Straughan [46], Singh [47], Singh and Tomar [48]. Passarella [49] introduced a theory of micropolar thermoelasticity for materials with voids based on the Lebon [50] law for the heat conduction (hyperbolic-type heat equation). A theory of thermoelastic materials with voids without energy dissipation was presented by De Cicco and Diaco [51]. 


\subsection{On the theories of viscoelasticity and thermoviscoelasticity: Literature review}

Various theories of viscoelastic materials with voids of integral type have been proposed and a wide class of problems was studied by Cowin [52], Ciarletta and Scalia [53], Scalia [54], De Cicco and Nappa [55], and Martínez and Quintanilla [56]. In the last decade there are been interest in formulation of the mechanical theories of viscoelastic materials with voids of differential type. In this connection, Ieşan [57] has developed a nonlinear theory for a viscoelastic composite as a mixture of a porous elastic solid and a Kelvin-Voigt material. A linear variant of this theory was developed by Quintanilla [58], and existence and exponential decay of solutions were proved. A theory of thermoviscoelastic composites modelled as interacting Cosserat continua was presented by Ieşan [59]. Ieşan and Nappa [60] introduced a nonlinear theory of heat conducting mixtures where the individual components were modelled as Kelvin-Voigt viscoelastic materials. Some exponential decay estimates of solutions of equations of steady vibrations in the theory of viscoelastricity for Kelvin-Voigt materials were obtained by Chiriţă et al. [61].

In [62], Ieşan extends theory of elastic materials with voids (see Nunziato and Cowin $[29,30]$ ), the basic equations of the nonlinear theory of thermoviscoelasticity for Kelvin-Voigt materials with voids were established, the linearized version of this theory was derived, a uniqueness result and the continuous dependence of solution upon the initial data and supply terms were proved. The basic BVPs of steady vibrations in the linear theories of viscoelasticity and thermoviscoelasticity (see Ieşan [62]) for materials with voids were investigated by using potential method and the theory of singular integral equation in [1 - 4]. This method was also developed in the classical theories of viscoelasticity and thermoviscoelasticity for Kelvin-Voigt materials without voids and the uniqueness and existence theorems were proved by Svanadze [63 - 65].

Recently, the theory of thermoviscoelasticity for Kelvin-Voigt microstretch composite materials was presented by Passarella et al. [66]. The propagation of plane harmonic waves in an isotropic generalized thermoviscoelastic medium with voids is studied and the fundamental solution of system of differential equations in the theory of generalized thermoviscoelasticity with voids is constructed by Sharma and Kumar [67], Tomar et al. [68].

An account of the historical developments of the theory of porous media as 


\subsection{On the theories of viscoelasticity and thermoviscoelasticity: Literature review}

well as references to various contributions may be found in the books by de Boer [69], Ieşan [70], Straughan [71, 72] and the references therein. A new approach may be found in Amendola et al. [8], although this is not limited just to voids.

The investigation of the BVPs of mathematical physics by the classical potential method has more that a hundred year history. The application of this method to the 3D (2D) basic BVPs of mathematical physics and the theory of elasticity reduces these problems to 2D (1D) integral equations. In mathematical physics the Dirichlet, Neumann, Robin and mixed type BVPs were reduced to the equivalent Fredholms integral equations by the virtue of the harmonic potentials (for details, see Kellogg [73], Günther [74], Hsiao and Wendland [75], Cheng and Cheng [76]). The existence theorems for the internal and external BVPs were proved by Fredholm's [77] theory of integral equations.

In the beginning of the 20th century the basic BVPs of the classical theory of elasticity were reduced to the equivalent integral equations by using the elastopotentials. The boundary integrals were strongly singular and need to be defined in terms of Cauchy principal value integrals. It was necessary to construct the theory of $1 \mathrm{D}$ and multidimensional singular integral equations for proof the existence theorems by potential method.

The corresponding potentials were constructed and applied to BVPs of the classical theory of elasticity in the works of representatives of the Italian mathematical school (E. Betti, T. Boggio, G. Lauricella, R. Marcolongo, F. Tricomi, V. Volterra and others). The main results in this subject are obtained by J. Boussinesq, K. Korn, H. Weyl, H. Poincaré, Georgian scientists (N. Muskhelishvili, I. Vekua, V. Kupradze, T. Gegelia, M. Basheleishvili, T. Burchuladze) and others.

Indeed, Muskhelishvili [78, 79] developed the theory of 1D singular integral equations and, using this theory, studied plane BVPs of the classical theory of elasticity. Vekua [80] presented the general methods of construction of the Shell theory. Owing to the works of Mikhlin [81], Kupradze [82], Kupradze et al. [83], and Burchuladze and Gegelia [84], the theory of multidimensional singular integral equations has presently been worked out with sufficient completeness.

In the 60ies of the 20th century singular potentials had been studied completely by A. Calderon, A. Zygmund, F. Tricomi, G. Giraud, T. Gegelia and others, and the existence of solutions of the basic BVPs of the 3D classical the- 
ory of elasticity was proved by the potential method. Then, in the 70ies, the dynamical and contact problems of the classical theories of elasticity and thermoelasticity were studied completely by the Georgian mathematicians led by V. Kupradze (for details, see Kupradze et al. [83], and Burchuladze and Gegelia [84] and references therein). An extensive review of works on the potential method can be found in the survey paper by Gegelia and Jentsch [85].

In the next chapters the basic BVPs of the linear theories of viscoelasticity and thermoviscoelasticity for Kelvin-Voigt materials with voids are investigated by using the potential method and the theory of singular integral equations.

\subsection{Basic notations}

Each chapter has its own numeration of formulas. The formula number is denoted by two figures enclosed in brackets; for example, (3.2) means the second formula in the third chapter. Theorems, lemmas, definitions and remarks are numerated in the same manner but without brackets; for example, theorem 3.2 means the second theorem in the third chapter.

We denote the vectors (vectors fields), matrices (matrices fields) and points of the Euclidean three-dimensional space $\mathbb{R}^{3}$ by boldface letter, and scalars (scalar fields) by Italic lightface letters.

Let $\mathbf{x}=\left(x_{1}, x_{2}, x_{3}\right)$ be a point of $\mathbb{R}^{3}$, the $t$ denotes the time variable, $t \geq 0$, $\mathbf{D}_{\mathbf{x}}=\left(\frac{\partial}{\partial x_{1}}, \frac{\partial}{\partial x_{2}}, \frac{\partial}{\partial x_{3}}\right)$; the nabla (gradient) and the Laplacian operators will be designated by $\nabla$ and $\Delta$, respectively; $\delta_{l j}$ and $\delta(\mathbf{x})$ denote the Kronecker's and Dirac delta, respectively; the unit matrices will always denote by $\mathbf{I}=\left(\delta_{l j}\right)_{3 \times 3}$, $\mathbf{J}=\left(\delta_{l j}\right)_{4 \times 4}$ and $\mathbf{J}^{\prime}=\left(\delta_{l j}\right)_{5 \times 5}$.

The inner (scalar) product of two vectors $\mathbf{w}=\left(w_{1}, w_{2}, \cdots, w_{l}\right)$ and $\mathbf{v}=$ $\left(v_{1}, v_{2}, \cdots, v_{l}\right)$ is denoted by $\mathbf{w} \cdot \mathbf{v}=\sum_{j=1}^{l} w_{j} \bar{v}_{j}$, where $\bar{v}_{j}$ is the complex conjugate of $v_{j}$. If $l=3$, then the vector product of vectors $\mathbf{w}$ and $\mathbf{v}$ is denoted by $[\mathbf{w} \times \mathbf{v}]$.

We consider an isotropic homogeneous viscoelastic Kelvin-Voigt material with voids that occupies the region $\Omega$ of $\mathbb{R}^{3}$. In the sequel we shall use the following notations from the theories of viscoelasticity and thermoviscoelasticity for KelvinVoigt materials with voids (see Ieşan [62]): 
$t_{l j}^{\prime}$ is the component of the total stress tensor;

$H_{j}^{\prime}$ is the component of the equilibrated stress;

$H_{0}^{\prime}$ is the intrinsic equilibrated body force;

$\mathbf{u}^{\prime}=\left(u_{1}^{\prime}, u_{2}^{\prime}, u_{3}^{\prime}\right)$ and $\mathbf{u}=\left(u_{1}, u_{2}, u_{3}\right)$ are the displacement vectors;

$\varphi^{\prime}$ and $\varphi$ are the volume fraction fields;

$\mathcal{F}^{\prime}=\left(\mathcal{F}_{1}^{\prime}, \mathcal{F}_{2}^{\prime}, \mathcal{F}_{3}^{\prime}\right)$ and $\mathcal{F}=\left(\mathcal{F}_{1}, \mathcal{F}_{2}, \mathcal{F}_{3}\right)$ are the body forces per unit mass;

$\mathcal{F}_{4}^{\prime}$ and $\mathcal{F}_{4}$ are the extrinsic equilibrated body forces per unit mass;

$\mathcal{F}_{5}^{\prime}$ and $\mathcal{F}_{5}$ are the heat supply per unit mass and unit time;

$\eta^{\prime}$ is the entropy per unit mass and unit time;

$Q_{j}^{\prime}$ is the component of heat flux vector;

$e_{l j}^{\prime}$ is the component of the strain tensor;

$\rho$ is the reference mass density, $\rho>0$;

$\kappa^{\prime}$ is the equilibrated inertia, $\kappa^{\prime}>0, \rho_{0}=\rho \kappa^{\prime}$;

$\omega$ is the oscillation (angular) frequency, $\omega>0$;

$T_{0}$ is the constant absolute temperature of the body in the reference configuration,

$T_{0}>0 ;$

$\theta^{\prime}$ and $\theta$ are the temperatures measured from $T_{0}$;

$\lambda, \lambda^{*}, \mu, \mu^{*}, b, b^{*}, \alpha, \alpha^{*}, \xi, \xi^{*}, \nu^{*}, k, \tau^{*}, m, a, \beta, \zeta^{*}$ are the constitutive coefficients.

Throughout this thesis, we employ the Einstein summation convention ac-

cording to which summation over the range 1,2, 3 is implied for any index that is repeated twice in any term, a subscript preceded by a comma denotes partial differentiation with respect to the corresponding Cartesian coordinate, and a superposed dot denotes differentiation with respect to $t$, so that, for instance,

$$
\begin{aligned}
& \varphi_{, j}=\frac{\partial \varphi}{\partial x_{j}}, \quad \varphi_{, j j}=\sum_{j=1}^{3} \frac{\partial^{2} \varphi}{\partial x_{j}^{2}}=\Delta \varphi \\
& \dot{\varphi}^{\prime}(\mathbf{x}, t)=\frac{\partial \varphi^{\prime}(\mathbf{x}, t)}{\partial t}, \quad \ddot{\varphi}^{\prime}(\mathbf{x}, t)=\frac{\partial^{2} \varphi^{\prime}(\mathbf{x}, t)}{\partial t^{2}} .
\end{aligned}
$$

Let $S$ be the smooth closed surface surrounding the finite domain $\Omega^{+}$in $\mathbb{R}^{3}, \bar{\Omega}^{+}=\Omega^{+} \cup S, \Omega^{-}=\mathbb{R}^{3} \backslash \bar{\Omega}^{+}, \bar{\Omega}^{-}=\Omega^{-} \cup S$.

A vector function $\mathbf{U}=\left(U_{1}, U_{2}, \cdots, U_{l}\right)$ is called regular in $\Omega^{-}\left(\right.$or $\left.\Omega^{+}\right)$if 
1)

$$
U_{j} \in C^{2}\left(\Omega^{-}\right) \cap C^{1}\left(\bar{\Omega}^{-}\right) \quad\left(\text { or } \quad U_{j} \in C^{2}\left(\Omega^{+}\right) \cap C^{1}\left(\bar{\Omega}^{+}\right)\right),
$$

2)

$$
U_{j}(\mathbf{x})=O\left(|\mathbf{x}|^{-1}\right), \quad U_{j, r}(\mathbf{x})=o\left(|\mathbf{x}|^{-1}\right) \quad \text { for } \quad|\mathbf{x}| \gg 1,
$$

where $j=1,2, \cdots, l$ and $r=1,2,3$.

In the Chapters 2 and 3 (Chapters 4 and 5) we consider a class of fourcomponent (five-component) regular vectors. 


\section{Chapter 2}

\section{Solutions of equations in the theory of viscoelasticity for materials with voids}

\subsection{Basic equations}

The theory of elasticity for solids with voids (see Nunziato and Cowin [29, 30]) is extended by Ieşan [62] to the case when the time derivative of the strain tensor and the time derivative of the gradient of the volume fraction field are included in the set of independent constitutive variables. The complete system of field equations in the linear theory of viscoelasticity for Kelvin-Voigt material with voids consists of the following equations (Ieşan [62]):

1) The equations of motion

$$
t_{l j, j}^{\prime}=\rho\left(\ddot{u}_{l}^{\prime}-\mathcal{F}_{l}^{\prime}\right)
$$

and

$$
H_{j, j}^{\prime}+H_{0}^{\prime}=\rho_{0} \ddot{\varphi}^{\prime}-\rho \mathcal{F}_{4}^{\prime}, \quad l=1,2,3 ;
$$


2) The constitutive equations

$$
\begin{aligned}
& t_{l j}^{\prime}=2 \mu e_{l j}^{\prime}+\lambda e_{r r}^{\prime} \delta_{l j}+b \varphi^{\prime} \delta_{l j}+2 \mu^{*} \dot{e}_{l j}^{\prime}+\lambda^{*} \dot{e}_{r r}^{\prime} \delta_{l j}+b^{*} \dot{\varphi}^{\prime} \delta_{l j}, \\
& H_{j}^{\prime}=\alpha \varphi_{, j}^{\prime}+\alpha^{*} \dot{\varphi}_{, j}^{\prime}, \\
& H_{0}^{\prime}=-b e_{r r}^{\prime}-\xi \varphi^{\prime}-\nu^{*} \dot{e}_{r r}^{\prime}-\xi^{*} \dot{\varphi}^{\prime}, \quad l, j=1,2,3
\end{aligned}
$$

3) The geometrical equations

$$
e_{l j}^{\prime}=\frac{1}{2}\left(u_{l, j}^{\prime}+u_{j, l}^{\prime}\right), \quad l, j=1,2,3 .
$$

Substituting (2.3) and (2.4) into (2.1) and (2.2), we obtain the following system of equations of motion in the linear theory of viscoelasticity for Kelvin-Voigt materials with voids expressed in terms of the displacement vector $\mathbf{u}^{\prime}$ and the volume fraction field $\varphi^{\prime}$ (Ieşan [62])

$$
\begin{aligned}
& \mu \Delta \mathbf{u}^{\prime}+(\lambda+\mu) \nabla \operatorname{div} \mathbf{u}^{\prime}+b \nabla \varphi^{\prime}+\mu^{*} \Delta \dot{\mathbf{u}}^{\prime}+\left(\lambda^{*}+\mu^{*}\right) \nabla \operatorname{div} \dot{\mathbf{u}}^{\prime}+b^{*} \nabla \dot{\varphi}^{\prime} \\
& =\rho\left(\ddot{\mathbf{u}}^{\prime}-\mathcal{F}^{\prime}\right), \\
& (\alpha \Delta-\xi) \varphi^{\prime}-b \operatorname{div} \mathbf{u}^{\prime}+\left(\alpha^{*} \Delta-\xi^{*}\right) \dot{\varphi}^{\prime}-\nu^{*} \operatorname{div} \dot{\mathbf{u}}^{\prime} \\
& =\rho_{0} \ddot{\varphi}^{\prime}-\rho \mathcal{F}_{4}^{\prime} .
\end{aligned}
$$

If the displacement vector $\mathbf{u}^{\prime}$ and the volume fraction function $\varphi^{\prime}$, the body force $\mathcal{F}^{\prime}$ and the extrinsic equilibrated body force $\mathcal{F}_{4}^{\prime}$ are postulated to have a harmonic time variation, that is,

$$
\left\{\mathbf{u}^{\prime}, \varphi^{\prime}, \mathcal{F}^{\prime}, \mathcal{F}_{4}^{\prime}\right\}(\mathbf{x}, t)=\operatorname{Re}\left[\left\{\mathbf{u}, \varphi, \mathcal{F}, \mathcal{F}_{4}\right\}(\mathbf{x}) e^{-i \omega t}\right]
$$

then from system of equations of motion (2.5) we obtain the following system of equations of steady vibrations in the linear theory of viscoelasticity for Kelvin- 
Voigt materials with voids

$$
\begin{aligned}
& \mu_{1} \Delta \mathbf{u}+\left(\lambda_{1}+\mu_{1}\right) \nabla \operatorname{div} \mathbf{u}+b_{1} \nabla \varphi+\rho \omega^{2} \mathbf{u}=-\rho \mathcal{F} \\
& \left(\alpha_{1} \Delta+\xi_{2}\right) \varphi-\nu_{1} \operatorname{div} \mathbf{u}=-\rho \mathcal{F}_{4}
\end{aligned}
$$

where

$$
\begin{array}{ll}
\lambda_{1}=\lambda-i \omega \lambda^{*}, & \mu_{1}=\mu-i \omega \mu^{*}, \quad b_{1}=b-i \omega b^{*}, \\
\alpha_{1}=\alpha-i \omega \alpha^{*}, & \nu_{1}=b-i \omega \nu^{*}, \\
\xi_{1}=\xi-i \omega \xi^{*}, & \xi_{2}=\rho_{0} \omega^{2}-\xi_{1} .
\end{array}
$$

Obviously, (2.6) is the system of partial differential equations with complex coefficients in which are 14 real parameters: $\lambda, \lambda^{*}, \mu, \mu^{*}, b, b^{*}, \alpha, \alpha^{*}, \xi, \xi^{*}, \nu^{*}, \omega, \rho$ and $\rho_{0}$.

We introduce the matrix differential operator

$$
\begin{aligned}
& \mathbf{A}\left(\mathbf{D}_{\mathbf{x}}\right)=\left(A_{p q}\left(\mathbf{D}_{\mathbf{x}}\right)\right)_{4 \times 4}, \\
& A_{l j}\left(\mathbf{D}_{\mathbf{x}}\right)=\left(\mu_{1} \Delta+\rho \omega^{2}\right) \delta_{l j}+\left(\lambda_{1}+\mu_{1}\right) \frac{\partial^{2}}{\partial x_{l} \partial x_{j}}, \\
& A_{l 4}\left(\mathbf{D}_{\mathbf{x}}\right)=b_{1} \frac{\partial}{\partial x_{l}}, \quad A_{4 l}\left(\mathbf{D}_{\mathbf{x}}\right)=-\nu_{1} \frac{\partial}{\partial x_{l}}, \\
& A_{44}\left(\mathbf{D}_{\mathbf{x}}\right)=\alpha_{1} \Delta+\xi_{2}, \quad l, j=1,2,3 .
\end{aligned}
$$

The system (2.6) can be written as

$$
\mathbf{A}\left(\mathbf{D}_{\mathbf{x}}\right) \mathbf{U}(\mathbf{x})=\mathbf{F}
$$

where $\mathbf{U}=(\mathbf{u}, \varphi)$ and $\mathbf{F}=\left(-\rho \mathcal{F},-\rho \mathcal{F}_{4}\right)$ are the four-component vector functions and $\mathbf{x} \in \Omega$.

Obviously, if $\mathbf{F}=\mathbf{0}$, then from (2.6) and (2.9) we obtain the following homo- 
geneous equations

$$
\begin{aligned}
& \mu_{1} \Delta \mathbf{u}+\left(\lambda_{1}+\mu_{1}\right) \nabla \operatorname{div} \mathbf{u}+b_{1} \nabla \varphi+\rho \omega^{2} \mathbf{u}=\mathbf{0} \\
& \left(\alpha_{1} \Delta+\xi_{2}\right) \varphi-\nu_{1} \operatorname{div} \mathbf{u}=0
\end{aligned}
$$

and

$$
\mathbf{A}\left(\mathbf{D}_{\mathbf{x}}\right) \mathbf{U}(\mathbf{x})=\mathbf{0}
$$

respectively.

Throughout this chapter, we suggest that $\xi_{2} \neq 0$ (the case $\xi_{2}=0$ is to simple to be considered).

\subsection{Solution of the dispersion equations. Plane harmonic waves}

We introduce the notations

$$
\begin{aligned}
& \mu_{0}=\lambda+2 \mu, \quad \mu_{0}^{*}=\lambda^{*}+2 \mu^{*}, \quad \mu_{2}=\mu_{0}-i \omega \mu_{0}^{*}, \\
& \xi_{0}=\rho_{0} \omega^{2}-\xi, \quad d^{*}=4 \mu_{0}^{*} \xi^{*}-\left(b^{*}+\nu^{*}\right)^{2}, \\
& d=\mu_{0}^{*} \xi^{*}-b^{*} \nu^{*}=\frac{1}{4}\left[d^{*}+\left(b^{*}-\nu^{*}\right)^{2}\right], \quad a_{1}=b^{2}+\omega^{2} d, \\
& a_{2}=b\left(b^{*}+\nu^{*}\right), \quad a_{3}=\omega^{2} \alpha^{*} \mu_{0}^{*}, \quad a_{4}=\alpha^{*} a_{1}+\xi^{*} a_{3} .
\end{aligned}
$$

In this section, it is assumed that

$$
\mu^{*}>0, \quad \mu_{0}^{*}>0, \quad \alpha^{*}>0, \quad d^{*}>0 .
$$

On the basis of (2.13) from (2.12) we get

$$
\mu_{0}^{*}>0, \quad \xi^{*}>0, \quad d>0, \quad a_{1}>0, \quad a_{3}>0, \quad a_{4}>0 .
$$


Suppose that plane harmonic waves corresponding to the wave number $\tau$ and angular frequency $\omega$ are propagated in the $x_{1}$-direction through the viscoelastic Kelvin-Voigt material with voids. Then

$$
\begin{aligned}
& \mathbf{u}^{\prime}(\mathbf{x}, t)=\mathbf{H} \exp \left\{i\left(\tau x_{1}-\omega t\right)\right\}, \\
& \varphi^{\prime}(\mathbf{x}, t)=H_{0} \exp \left\{i\left(\tau x_{1}-\omega t\right)\right\},
\end{aligned}
$$

where $\mathbf{H}=\left(H_{1}, H_{2}, H_{3}\right) ; H_{0}, H_{1}, H_{2}$ and $H_{3}$ are constants.

Keeping in mind (2.15) from the system of homogeneous equations of motion of the linear theory of viscoelasticity for Kelvin-Voigt materials with voids (see $(2.5))$

$$
\begin{aligned}
& \mu \Delta \mathbf{u}^{\prime}+(\lambda+\mu) \nabla \operatorname{div} \mathbf{u}^{\prime}+b \nabla \varphi^{\prime}+\mu^{*} \Delta \dot{\mathbf{u}}^{\prime}+\left(\lambda^{*}+\mu^{*}\right) \nabla \operatorname{div} \dot{\mathbf{u}}^{\prime} \\
& \quad+b^{*} \nabla \dot{\varphi}^{\prime}-\rho \ddot{\mathbf{u}}=\mathbf{0}, \\
& (\alpha \Delta-\xi) \varphi^{\prime}-b \operatorname{div} \mathbf{u}^{\prime}+\left(\alpha^{*} \Delta-\xi^{*}\right) \dot{\varphi}^{\prime}-\nu^{*} \operatorname{div} \dot{\mathbf{u}}^{\prime}-\rho_{0} \ddot{\varphi}^{\prime}=0
\end{aligned}
$$

it follows that

$$
\begin{aligned}
& \left\{\left[\mu_{1}+\left(\lambda_{1}+\mu_{1}\right) \delta_{1 l}\right] \tau^{2}-\rho \omega^{2}\right\} H_{l}-i \tau b_{1} \delta_{1 l} H_{0}=0, \\
& i \tau \nu_{1} H_{1}+\left(\alpha_{1} \tau^{2}-\xi_{2}\right) H_{0}=0, \quad l=1,2,3 .
\end{aligned}
$$

From (2.16) for $H_{0}$ and $H_{1}$ we have

$$
\begin{aligned}
& \left(\mu_{2} \tau^{2}-\rho \omega^{2}\right) H_{1}-i \tau b_{1} H_{0}=0, \\
& i \tau \nu_{1} H_{1}+\left(\alpha_{1} \tau^{2}-\xi_{2}\right) H_{0}=0 .
\end{aligned}
$$

For the system (2.17) to have a non-trivial solution for $H_{0}$ and $H_{1}$ we must set the determinant of its coefficients equal to zero, thus

$$
\alpha_{1} \mu_{2} \tau^{4}-\left(\xi_{2} \mu_{2}+\rho \omega^{2} \alpha_{1}+b_{1} \nu_{1}\right) \tau^{2}+\rho \omega^{2} \xi_{2}=0
$$


In the same way from (2.16) for $H_{2}$ and $H_{3}$ we have

$$
\left(\mu_{1} \tau^{2}-\rho \omega^{2}\right) H_{l}=0, \quad l=2,3,
$$

and if $\tau$ is the solution of equation

$$
\mu_{1} \tau^{2}-\rho \omega^{2}=0
$$

then (2.19) has a non-trivial solution.

The relations (2.18) and (2.20) will be called the dispersion equations of longitudinal and transverse plane waves in the linear theory of viscoelasticity for Kelvin-Voigt materials with voids, respectively. It is obvious that if $\tau>0$, then the corresponding plane wave has the constant amplitude, and if $\tau$ is complex with $\operatorname{Im} \tau>0$, then the plane wave is attenuated as $x_{1} \rightarrow+\infty$ (see Achenbach [86]).

Let $\tau_{1}^{2}, \tau_{2}^{2}$ and $\tau_{3}^{2}$ be roots of equations (2.18) and (2.20) with respect to $\tau^{2}$, respectively. Obviously,

$$
\tau_{3}^{2}=\frac{\rho \omega^{2}}{\mu_{1}}
$$

One may easily verify that $\tau_{3}^{2}$ is a complex number. Obviously, $\tau_{1}, \tau_{2}$ and $\tau_{3}$ are the wave numbers of longitudinal and transverse plane harmonic waves, respectively.

We denote the longitudinal plane wave with wave number $\tau_{j}(j=1,2)$ by $P_{j}$ (P-primary), and the transverse horizontal and vertical plane waves with wave number $\tau_{3}$ by $S H$ and $S V$, respectively (S-secondary, see Achenbach [86]).

Lemma 2.1. If the conditions (2.13) are satisfied, then the equation (2.18) with respect to $\tau^{2}$ has not a positive root.

Proof. Let $\eta$ be a real root of the equation

$$
\alpha_{1} \mu_{2} \eta^{2}-\left(\xi_{2} \mu_{2}+\rho \omega^{2} \alpha_{1}+b_{1} \nu_{1}\right) \eta+\rho \omega^{2} \xi_{2}=0
$$

Separating real and imaginary parts in (2.21), on the basis of (2.7), (2.13) and 
equalities

$$
\begin{aligned}
& \alpha_{1} \mu_{2}=\alpha \mu_{0}-a_{3}-i \omega\left(\alpha \mu_{0}^{*}+\alpha^{*} \mu_{0}\right), \quad \rho \omega^{2} \xi_{2}=\xi_{0} \rho \omega^{2}+i \omega \xi^{*} \rho \omega^{2}, \\
& \xi_{2} \mu_{2}+\rho \omega^{2} \alpha_{1}+b_{1} \nu_{1}=\mu_{0} \xi_{0}+\alpha \rho \omega^{2}+a_{1}-i \omega\left(\mu_{0}^{*} \xi_{0}-\mu_{0} \xi^{*}+\alpha^{*} \rho \omega^{2}+a_{2}\right)
\end{aligned}
$$

we obtain the following system

$$
\begin{aligned}
& \left(\alpha \mu_{0}-a_{3}\right) \eta^{2}-\left(\mu_{0} \xi_{0}+\alpha \rho \omega^{2}+a_{1}\right) \eta+\xi_{0} \rho \omega^{2}=0 \\
& \left(\alpha \mu_{0}^{*}+\alpha^{*} \mu_{0}\right) \eta^{2}-\left(\mu_{0}^{*} \xi_{0}-\mu_{0} \xi^{*}+\alpha^{*} \rho \omega^{2}+a_{2}\right) \eta-\xi^{*} \rho \omega^{2}=0 .
\end{aligned}
$$

As one may easily verify, the system (2.22) may be written in the form

$$
\begin{gathered}
\eta_{1} \eta_{2}=\eta\left(a_{3} \eta+a_{1}\right), \\
\left(\alpha^{*} \eta+\xi^{*}\right) \eta_{1}=\eta\left(a_{2}-\mu_{0}^{*} \eta_{2}\right),
\end{gathered}
$$

where $\eta_{1}=\mu_{0} \eta-\rho \omega^{2}, \eta_{2}=\alpha \eta-\xi_{0}$. Obviously, by (2.23) and (2.24) we have $\eta \eta_{2} \neq 0$. On the other hand, taking into account (2.23) from (2.24) it follows that

$$
\left(\alpha^{*} \eta+\xi^{*}\right)\left(a_{3} \eta+a_{1}\right)=\eta_{2}\left(a_{2}-\mu_{0}^{*} \eta_{2}\right)
$$

and hence,

$$
\alpha^{*} a_{3} \eta\left(\eta-\eta_{0}\right)+\mu_{0}^{*} \eta_{2}^{2}-a_{2} \eta_{2}+a_{1} \xi^{*}=0
$$

where

$$
\eta_{0}=-\frac{\xi^{*} a_{3}+\alpha^{*} a_{1}}{\alpha^{*} a_{3}}<0 .
$$

By virtue of conditions (2.13) and (2.14) we have

$$
\alpha^{*} a_{3}>0, \quad a_{2}^{2}-4 a_{1} \mu_{0}^{*} \xi^{*}=-\left[b^{2} d^{*}+4 \mu_{0}^{*} d \omega^{2}\right]<0 .
$$

Therefore, from $(2.25)$ we obtain $\eta\left(\eta-\eta_{0}\right)<0$. Finally, we may write $\left.\eta \in\right] \eta_{0} ; 0[$ and lemma 2.1 is thereby proved. $\diamond$

We assume that $\operatorname{Im} \tau_{j}>0(j=1,2,3)$. Lemma 2.1 leads to the following 
result.

Theorem 2.1. If the conditions (2.13) are satisfied, then through a Kelvin-Voigt material with voids four plane harmonic plane waves propagate: two longitudinal plane waves $P_{1}$ and $P_{2}$ with wave numbers $\tau_{1}, \tau_{2}$ and two transverse plane waves $S H$ and $S V$ with wave number $\tau_{3}$; these are attenuated waves as $x_{1} \rightarrow+\infty$.

Remark 2.1. It is obvious that if plane harmonic waves are propagated in an arbitrary direction through a Kelvin-Voigt material with voids, then we obtain the same result as given in theorem 2.1.

Remark 2.2. In the theory of elasticity through an isotropic material with voids four (two longitudinal and two transverse) plane harmonic waves propagate; the longitudinal waves are attenuated and the transversal waves have the constant amplitudes (for details, see Puri and Cowin [32]).

\subsection{Fundamental solution}

Definition 2.1. The fundamental solution of the system (2.10) (the fundamental matrix of operator $\left.\mathbf{A}\left(\mathbf{D}_{\mathbf{x}}\right)\right)$ is the matrix $\boldsymbol{\Gamma}(\mathbf{x})=\left(\Gamma_{l j}(\mathbf{x})\right)_{4 \times 4}$ satisfying condition in the class of generalized functions (see Hörmander [87])

$$
\mathbf{A}\left(\mathbf{D}_{\mathbf{x}}\right) \boldsymbol{\Gamma}(\mathbf{x})=\delta(\mathbf{x}) \mathbf{J}
$$

where $\mathbf{x} \in \mathbb{R}^{3}$.

In what follows we assume that $\tau_{1}^{2} \neq \tau_{2}^{2} \neq \tau_{3}^{2} \neq \tau_{1}^{2}$. In the sequel we use the matrix differential operators: 
1)

$$
\begin{aligned}
\mathbf{L}\left(\mathbf{D}_{\mathbf{x}}\right)= & \left(L_{p q}\left(\mathbf{D}_{\mathbf{x}}\right)\right)_{4 \times 4}, \\
L_{l j}\left(\mathbf{D}_{\mathbf{x}}\right) & =\frac{1}{\mu_{1}}\left(\Delta+\tau_{1}^{2}\right)\left(\Delta+\tau_{2}^{2}\right) \delta_{l j} \\
& -\frac{1}{\alpha_{1} \mu_{1} \mu_{2}}\left[\left(\lambda_{1}+\mu_{1}\right)\left(\alpha_{1} \Delta+\xi_{2}\right)+b_{1} \nu_{1}\right] \frac{\partial^{2}}{\partial x_{l} \partial x_{j}}, \\
L_{l 4}\left(\mathbf{D}_{\mathbf{x}}\right) & =-\frac{b_{1}}{\alpha_{1} \mu_{1}} \frac{\partial}{\partial x_{l}}, \quad L_{4 l}\left(\mathbf{D}_{\mathbf{x}}\right)=\frac{\nu_{1}}{\alpha_{1} \mu_{1} \mu_{2}}\left(\mu_{1} \Delta+\rho \omega^{2}\right) \frac{\partial}{\partial x_{l}}, \\
L_{44}\left(\mathbf{D}_{\mathbf{x}}\right) & =\frac{1}{\alpha_{1} \mu_{1}}\left(\mu_{2} \Delta+\rho \omega^{2}\right), \quad l, j=1,2,3 .
\end{aligned}
$$

2)

$$
\begin{aligned}
& \Lambda(\Delta)=\left(L_{p q}(\Delta)\right)_{4 \times 4}, \\
& \Lambda_{11}(\Delta)=\Lambda_{22}(\Delta)=\Lambda_{33}(\Delta)=\left(\Delta+\tau_{1}^{2}\right)\left(\Delta+\tau_{2}^{2}\right)\left(\Delta+\tau_{3}^{2}\right), \\
& \Lambda_{44}(\Delta)=\left(\Delta+\tau_{1}^{2}\right)\left(\Delta+\tau_{2}^{2}\right), \quad \Lambda_{p q}(\Delta)=0, \\
& p, q=1,2,3,4, \quad p \neq q .
\end{aligned}
$$

We have the following result.

Lemma 2.2. If

$$
\alpha_{1} \mu_{1} \mu_{2} \neq 0,
$$

then

$$
\mathbf{A}\left(\mathbf{D}_{\mathbf{x}}\right) \mathbf{L}\left(\mathbf{D}_{\mathbf{x}}\right)=\boldsymbol{\Lambda}(\Delta) .
$$

Lemma 2.2 is proved by direct calculation. 
We introduce the notations

$$
\begin{aligned}
& \mathbf{Y}(\mathbf{x})=\left(Y_{p q}(\mathbf{x})\right)_{4 \times 4}, \quad Y_{11}(\mathbf{x})=Y_{22}(\mathbf{x})=Y_{33}(\mathbf{x})=c_{1 j} \gamma_{j}(\mathbf{x}), \\
& Y_{44}(\mathbf{x})=c_{2 j} \gamma_{j}(\mathbf{x}), \quad Y_{p q}(\mathbf{x})=0, \quad p, q=1,2,3,4, \quad p \neq q,
\end{aligned}
$$

where

$$
\begin{aligned}
& \gamma_{j}(\mathbf{x})=-\frac{e^{i \tau_{j}|\mathbf{x}|}}{4 \pi|\mathbf{x}|}, \quad c_{1 j}=\prod_{l=1 ; l \neq j}^{3}\left(\tau_{l}^{2}-\tau_{j}^{2}\right)^{-1}, \\
& c_{21}=-c_{22}=\left(\tau_{2}^{2}-\tau_{1}^{2}\right)^{-1}, \quad c_{23}=0, \quad j=1,2,3 .
\end{aligned}
$$

Obviously, $\mathbf{Y}$ is the fundamental matrix of operator $\boldsymbol{\Lambda}$, that is,

$$
\mathbf{\Lambda}(\Delta) \mathbf{Y}(\mathbf{x})=\delta(\mathbf{x}) \mathbf{J}
$$

where $\mathbf{x} \in \mathbb{R}^{3}$.

We define the matrix $\boldsymbol{\Gamma}=\left(\Gamma_{p q}\right)_{4 \times 4}$ by

$$
\Gamma(\mathbf{x})=\mathbf{L}\left(\mathbf{D}_{\mathbf{x}}\right) \mathbf{Y}(\mathbf{x})
$$

In view of (2.29), (2.31) and (2.32) we get

$$
\mathbf{A}\left(\mathbf{D}_{\mathbf{x}}\right) \boldsymbol{\Gamma}(\mathbf{x})=\mathbf{A}\left(\mathbf{D}_{\mathbf{x}}\right) \mathbf{L}\left(\mathbf{D}_{\mathbf{x}}\right) \mathbf{Y}(\mathbf{x})=\mathbf{\Lambda}(\Delta) \mathbf{Y}(\mathbf{x})=\delta(\mathbf{x}) \mathbf{J} .
$$

Hence, $\boldsymbol{\Gamma}(\mathbf{x})$ is the fundamental matrix of differential operator $\mathbf{A}\left(\mathbf{D}_{\mathbf{x}}\right)$. We have thereby proved the following theorem.

Theorem 2.2. If condition (2.28) is satisfied, then the matrix $\boldsymbol{\Gamma}(\mathbf{x})$ defined by (2.32) is the fundamental solution of system (2.10), where the matrices $\mathbf{L}\left(\mathbf{D}_{\mathbf{x}}\right)$ and $\mathbf{Y}$ are given by (2.26) and (2.30), respectively.

We are now in a position to establish basic properties of matrix $\boldsymbol{\Gamma}(\mathbf{x})$. Theorem 2.2 leads to the following results.

Corollary 2.1. If condition (2.28) is satisfied, then each column $\boldsymbol{\Gamma}^{(j)}(\mathbf{x})$ of the 
matrix $\mathbf{\Gamma}(\mathbf{x})$ is the solution of the homogeneous equation

$$
\mathbf{A}\left(\mathbf{D}_{\mathbf{x}}\right) \Gamma^{(j)}(\mathbf{x})=\mathbf{0}
$$

at every point $\mathbf{x} \in \mathbb{R}^{3}$ except the origin, where $\Gamma^{(j)}=\left(\Gamma_{1 j}, \Gamma_{2 j}, \Gamma_{3 j}\right)$ and $j=$ $1,2,3,4$.

Corollary 2.2. If condition (2.28) is satisfied, then the fundamental solution of the system

$$
\mu_{1} \Delta \mathbf{u}(\mathbf{x})+\left(\lambda_{1}+\mu_{1}\right) \nabla \operatorname{div} \mathbf{u}(\mathbf{x})=\mathbf{0}, \quad \alpha_{1} \Delta \varphi(\mathbf{x})=0
$$

is the matrix $\Psi=\left(\Psi_{p q}\right)_{4 \times 4}$, where

$$
\begin{aligned}
& \Psi_{l j}(\mathbf{x})=\left(\frac{1}{\mu_{1}} \Delta \delta_{l j}-\frac{\lambda_{1}+\mu_{1}}{\mu_{1} \mu_{2}} \frac{\partial^{2}}{\partial x_{l} \partial x_{j}}\right) \gamma_{4}(\mathbf{x}), \\
& \Psi_{l j}(\mathbf{x})=\frac{1}{\alpha_{1}} \gamma_{5}(\mathbf{x}), \quad \Psi_{l 4}(\mathbf{x})=\Psi_{4 j}(\mathbf{x})=0, \\
& \gamma_{4}(\mathbf{x})=-\frac{|\mathbf{x}|}{8 \pi}, \quad \gamma_{5}(\mathbf{x})=-\frac{1}{4 \pi|\mathbf{x}|}, \quad l, j=1,2,3 .
\end{aligned}
$$

Clearly (see Kupradze et al. [83]), the relations

$$
\begin{aligned}
& \Psi_{l j}(\mathbf{x})=O\left(|\mathbf{x}|^{-1}\right), \quad \Psi_{44}(\mathbf{x})=O\left(|\mathbf{x}|^{-1}\right) \\
& \Psi_{l 4}(\mathbf{x})=\Psi_{l 4}(\mathbf{x})=0, \quad l, j=1,2,3
\end{aligned}
$$

hold in a neighborhood of the origin.

On the basis of theorem 2.2 and corollary 2.2 we obtain the following result. 
Theorem 2.3. If condition (2.28) is satisfied, then the relations

$$
\begin{aligned}
& \Gamma_{l j}(\mathbf{x})=O\left(|\mathbf{x}|^{-1}\right), \quad \Gamma_{l j}(\mathbf{x})-\Psi_{l j}(\mathbf{x})=\text { const }+O(|\mathbf{x}|) \\
& \frac{\partial^{p}}{\partial x_{1}^{p_{1}} \partial x_{2}^{p_{2}} \partial x_{3}^{p_{3}}}\left[\Gamma_{l j}(\mathbf{x})-\Psi_{l j}(\mathbf{x})\right]=O\left(|\mathbf{x}|^{1-m}\right)
\end{aligned}
$$

hold in a neighborhood of the origin, where $p=p_{1}+p_{2}+p_{3}, p \geq 1, p_{q} \geq 0, q=$ $1,2,3$ and $l j=1,2,3,4$.

Thus, $\boldsymbol{\Psi}(\mathbf{x})$ is the singular part of the fundamental matrix $\mathbf{\Gamma}(\mathbf{x})$ in the neighborhood of the origin.

It is easy to see that each column $\boldsymbol{\Gamma}^{(j)}(\mathbf{x})$ of the matrix $\boldsymbol{\Gamma}(\mathbf{x})$ is represented as follows

$$
\boldsymbol{\Gamma}^{(j)}(\mathbf{x})=\sum_{l=1}^{3} \boldsymbol{\Gamma}^{(j l)}(\mathbf{x}),
$$

where $\Gamma^{(j l)}=\left(\Gamma_{1}^{(j l)}, \Gamma_{2}^{(j l)}, \Gamma_{3}^{(j l)}, \Gamma_{4}^{(j l)}\right) ; \Gamma_{p}^{(j l)}$ is a solution of Helmholtz equation

$$
\left(\Delta+\tau_{l}^{2}\right) \Gamma_{p}^{(j l)}(\mathbf{x})=0
$$

and satisfies the radiation conditions at infinity

$$
\begin{aligned}
& \Gamma_{p}^{(j l)}(\mathbf{x})=e^{i \tau_{l}|\mathbf{x}|} O\left(|\mathbf{x}|^{-1}\right) \\
& \left(\frac{\partial}{\partial|\mathbf{x}|}-i \tau_{l}\right) \Gamma_{p}^{(j l)}(\mathbf{x})=e^{i \tau_{l}|\mathbf{x}|} O\left(|\mathbf{x}|^{-2}\right)
\end{aligned}
$$

for $|\mathbf{x}| \gg 1, l=1,2,3, j, p=1,2,3,4$.

Equalities in (2.34) are the radiation conditions for metaharmonic functions (for details, see Sommerfeld [88], Kupradze [89], Vekua [90]).

Remark 2.3. For investigating BVPs of the theories of elasticity and thermoelasticity by potential method it is necessary to construct fundamental solutions of corresponding systems of partial differential equations and to establish their basic properties. Several methods are known for constructing fundamental solutions in the classical theories of elasticity and thermoelasticity (for details, see Gurtin 
[91], Hetnarski and Ignaczak [92], Nowacki [93]). The explicit expressions of fundamental solutions in the theory of elasticity, thermoelasticity and micropolar theory were obtained in different manner by Kupradze et al. [83], Sandru [94], Dragos [95]. The basic properties of fundamental solutions of partial differential equations are given in the book of Hörmander [87].

\subsection{Green's formulas. Representations of gen- eral solutions}

In this section, first, we establish the Green's formulas in the linear theory of viscoelasticity for Kelvin-Voigt materials with voids, then we obtain the integral representation of regular vector (representation of Somigliana-type) and the Galerkin-type solution of the system (2.6). Finally, we establish the representation of the general solution of the system of homogeneous equations (2.11) by using metaharmonic functions.

In the sequel we use the matrix differential operators $\tilde{\mathbf{A}}\left(\mathbf{D}_{\mathbf{x}}\right), \mathbf{P}\left(\mathbf{D}_{\mathbf{x}}, \mathbf{n}\right)$, $\tilde{\mathbf{P}}\left(\mathbf{D}_{\mathbf{x}}, \mathbf{n}\right)$, where $\tilde{\mathbf{A}}\left(\mathbf{D}_{\mathbf{x}}\right)=\mathbf{A}^{\top}\left(-\mathbf{D}_{\mathbf{x}}\right)$ and

$$
\begin{aligned}
& \mathbf{P}\left(\mathbf{D}_{\mathbf{x}}, \mathbf{n}\right)=\left(\mathbf{P}_{p q}\left(\mathbf{D}_{\mathbf{x}}, \mathbf{n}\right)\right)_{4 \times 4}, \quad \tilde{\mathbf{P}}\left(\mathbf{D}_{\mathbf{x}}, \mathbf{n}\right)=\left(\tilde{\mathbf{P}}_{p q}\left(\mathbf{D}_{\mathbf{x}}, \mathbf{n}\right)\right)_{4 \times 4} \\
& P_{l j}\left(\mathbf{D}_{\mathbf{x}}, \mathbf{n}\right)=\mu_{1} \delta_{l j} \frac{\partial}{\partial \mathbf{n}}+\mu_{1} n_{j} \frac{\partial}{\partial x_{l}}+\lambda_{1} n_{l} \frac{\partial}{\partial x_{j}} \\
& P_{l 4}\left(\mathbf{D}_{\mathbf{x}}, \mathbf{n}\right)=b_{1} n_{l}, \quad P_{4 l}\left(\mathbf{D}_{\mathbf{x}}, \mathbf{n}\right)=0, \quad P_{44}\left(\mathbf{D}_{\mathbf{x}}, \mathbf{n}\right)=\alpha_{1} \frac{\partial}{\partial \mathbf{n}} \\
& \tilde{P}_{p j}\left(\mathbf{D}_{\mathbf{x}}, \mathbf{n}\right)=P_{p j}\left(\mathbf{D}_{\mathbf{x}}, \mathbf{n}\right), \quad \tilde{P}_{j 4}\left(\mathbf{D}_{\mathbf{x}}, \mathbf{n}\right)=\nu_{1} n_{j}, \\
& \tilde{P}_{44}\left(\mathbf{D}_{\mathbf{x}}, \mathbf{n}\right)=P_{44}\left(\mathbf{D}_{\mathbf{x}}, \mathbf{n}\right), \quad l, j=1,2,3, \quad p=1,2,3,4 .
\end{aligned}
$$

Here $\mathbf{n}=\left(n_{1}, n_{2}, n_{3}\right)$ is the unit vector, $\frac{\partial}{\partial \mathbf{n}}$ is the derivative along the vector $\mathbf{n}$ and the superscript $\top$ denotes transposition.

Obviously, the fundamental matrix $\tilde{\boldsymbol{\Gamma}}(\mathbf{x})$ of operator $\tilde{\mathbf{A}}\left(\mathbf{D}_{\mathbf{x}}\right)$ satisfies the fol- 
lowing condition

$$
\tilde{\Gamma}(\mathbf{x})=\Gamma^{\top}(-\mathbf{x}) .
$$

Let $\tilde{\mathbf{U}}_{j}$ be the $j$-th column of the matrix $\tilde{\mathbf{U}}=\left(\tilde{U}_{l j}\right)_{4 \times 4}, j=1,2,3,4$. As in classical theory of thermoelasticity (for details, see Kupradze et al. [83]) we can prove the following result.

Theorem 2.4. If $U$ and $\tilde{U}_{j}(j=1,2,3,4)$ are regular vectors in $\Omega^{+}$, then

$$
\begin{aligned}
& \int_{\Omega^{+}}\left\{\left[\tilde{\mathbf{A}}\left(\mathbf{D}_{\mathbf{y}}\right) \tilde{\mathbf{U}}(\mathbf{y})\right]^{\top} \mathbf{U}(\mathbf{y})-[\tilde{\mathbf{U}}(\mathbf{y})]^{\top} \mathbf{A}\left(\mathbf{D}_{\mathbf{y}}\right) \mathbf{U}(\mathbf{y})\right\} d \mathbf{y} \\
& =\int_{S}\left\{\left[\tilde{\mathbf{P}}\left(\mathbf{D}_{\mathbf{z}}, \mathbf{n}\right) \tilde{\mathbf{U}}(\mathbf{z})\right]^{\top} \mathbf{U}(\mathbf{z})-[\tilde{\mathbf{U}}(\mathbf{z})]^{\top} \mathbf{P}\left(\mathbf{D}_{\mathbf{z}}, \mathbf{n}\right) \tilde{\mathbf{U}}(\mathbf{z})\right\} d_{z} S .
\end{aligned}
$$

On the basis of theorem 2.4 and condition (1.1) we obtain the following result.

Theorem 2.5. If $U$ and $\tilde{U}_{j}(j=1,2,3,4)$ are regular vectors in $\Omega^{-}$, then

$$
\begin{aligned}
& \int_{\Omega^{-}}\left\{\left[\tilde{\mathbf{A}}\left(\mathbf{D}_{\mathbf{y}}\right) \tilde{\mathbf{U}}(\mathbf{y})\right]^{\top} \mathbf{U}(\mathbf{y})-[\tilde{\mathbf{U}}(\mathbf{y})]^{\top} \mathbf{A}\left(\mathbf{D}_{\mathbf{y}}\right) \mathbf{U}(\mathbf{y})\right\} d \mathbf{y} \\
& =-\int_{S}\left\{\left[\tilde{\mathbf{P}}\left(\mathbf{D}_{\mathbf{z}}, \mathbf{n}\right) \tilde{\mathbf{U}}(\mathbf{z})\right]^{\top} \mathbf{U}(\mathbf{z})-[\tilde{\mathbf{U}}(\mathbf{z})]^{\top} \mathbf{P}\left(\mathbf{D}_{\mathbf{z}}, \mathbf{n}\right) \tilde{\mathbf{U}}(\mathbf{z})\right\} d_{z} S .
\end{aligned}
$$

The identities (2.37) and (2.38) are the Green's formulas in the linear theory of viscoelasticity for Kelvin-Voigt materials with voids for domains $\Omega^{+}$and $\Omega^{-}$, respectively.

Keeping in mind (2.33), (2.34), (2.36), theorems 2.2 and 2.3 from (2.37) and (2.38) we obtain the formulas of integral representation of regular vector (representation of Somigliana-type) for the domains $\Omega^{+}$and $\Omega^{-}$. 
Theorem 2.6. If $U$ is a regular vector in $\Omega^{+}$, then

$$
\begin{aligned}
\mathbf{U}(\mathbf{x})= & \int_{S}\left\{\left[\tilde{\mathbf{P}}\left(\mathbf{D}_{\mathbf{z}}, \mathbf{n}\right) \boldsymbol{\Gamma}^{\top}(\mathbf{x}-\mathbf{z})\right]^{\top} \mathbf{U}(\mathbf{z})-\boldsymbol{\Gamma}(\mathbf{x}-\mathbf{z}) \mathbf{P}\left(\mathbf{D}_{\mathbf{z}}, \mathbf{n}\right) \mathbf{U}(\mathbf{z})\right\} d_{\mathbf{z}} S \\
& +\int_{\Omega^{+}} \boldsymbol{\Gamma}(\mathbf{x}-\mathbf{y}) \mathbf{A}\left(\mathbf{D}_{\mathbf{y}}\right) \mathbf{U}(\mathbf{y}) d \mathbf{y}
\end{aligned}
$$

Theorem 2.7. If $U$ is a regular vector in $\Omega^{-}$, then

$$
\begin{aligned}
\mathbf{U}(\mathbf{x})= & -\int_{S}\left\{\left[\tilde{\mathbf{P}}\left(\mathbf{D}_{\mathbf{z}}, \mathbf{n}\right) \boldsymbol{\Gamma}^{\top}(\mathbf{x}-\mathbf{z})\right]^{\top} \mathbf{U}(\mathbf{z})-\boldsymbol{\Gamma}(\mathbf{x}-\mathbf{z}) \mathbf{P}\left(\mathbf{D}_{\mathbf{z}}, \mathbf{n}\right) \mathbf{U}(\mathbf{z})\right\} d d_{\mathbf{z}} S \\
& +\int_{\Omega^{-}} \boldsymbol{\Gamma}(\mathbf{x}-\mathbf{y}) \mathbf{A}\left(\mathbf{D}_{\mathbf{y}}\right) \mathbf{U}(\mathbf{y}) d \mathbf{y}
\end{aligned}
$$

The next two theorems provide a Galerkin-type solution to system (2.6).

Theorem 2.8. Let

$$
\begin{aligned}
\mathbf{u}(\mathbf{x})= & \frac{1}{\mu_{1}}\left(\Delta+\tau_{1}^{2}\right)\left(\Delta+\tau_{2}^{2}\right) \mathbf{w}(\mathbf{x}) \\
& -\frac{1}{\alpha_{1} \mu_{1} \mu_{2}}\left[\left(\lambda_{1}+\mu_{1}\right)\left(\alpha_{1} \Delta+\xi_{2}\right)+b_{1} \nu_{1}\right] \nabla \operatorname{div} \mathbf{w}(\mathbf{x}) \\
& -\frac{b_{1}}{\alpha_{1} \mu_{1}} \nabla w_{0}(\mathbf{x}) \\
\varphi(\mathbf{x})= & \frac{\nu_{1}}{\alpha_{1} \mu_{1} \mu_{2}}\left(\mu_{1} \Delta+\rho \omega^{2}\right) \operatorname{div} \mathbf{w}(\mathbf{x})+\frac{1}{\alpha_{1} \mu_{1}}\left(\mu_{2} \Delta+\rho \omega^{2}\right) w_{0}(\mathbf{x})
\end{aligned}
$$


where $\mathbf{w}=\left(w_{1}, w_{2}, w_{3}\right) \in C^{6}(\Omega), w_{0} \in C^{4}(\Omega)$, and

$$
\begin{aligned}
& \left(\Delta+\tau_{1}^{2}\right)\left(\Delta+\tau_{2}^{2}\right)\left(\Delta+\tau_{3}^{2}\right) \mathbf{w}(\mathbf{x})=-\rho \mathcal{F}(\mathbf{x}), \\
& \left(\Delta+\tau_{1}^{2}\right)\left(\Delta+\tau_{2}^{2}\right) w_{0}(\mathbf{x})=-\rho \mathcal{F}_{4}(\mathbf{x})
\end{aligned}
$$

then $\mathbf{U}=(\mathbf{u}, \varphi)$ is a solution of system (2.6).

Proof. By virtue of (2.26) and (2.27) the equations (2.41) and (2.42) we can rewrite in the form

$$
\mathbf{U}(\mathbf{x})=\mathbf{L}\left(\mathbf{D}_{\mathbf{x}}\right) \mathbf{W}(\mathbf{x})
$$

and

$$
\mathbf{\Lambda}(\Delta) \mathbf{W}(\mathbf{x})=\mathbf{F}(\mathbf{x})
$$

respectively, where $\mathbf{W}=\left(\mathbf{w}, w_{0}\right), \mathbf{F}(\mathbf{x})=\left(-\rho \mathcal{F},-\rho \mathcal{F}_{4}\right)$. Clearly, by (2.29), (2.43) and (2.44) the vector $\mathbf{U}$ is a solution of the system (2.6).

Theorem 2.9. If $\mathbf{U}=(\mathbf{u}, \varphi)$ is a solution of system (2.6) in $\Omega$, then $\mathbf{U}$ is represented by (2.41), where $\mathbf{W}=\left(\mathbf{w}, w_{0}\right)$ is a solution of (2.42) and $\Omega$ is a finite domain in $R^{3}$.

Proof. Let $\mathbf{U}$ be a solution of system (2.6). Obviously, if $\Psi^{\prime}(\mathbf{x})$ is the fundamental matrix of the operator $\mathbf{L}\left(\mathbf{D}_{\mathbf{x}}\right)$ (see $(2.26)$ ), then the vector function

$$
\mathbf{W}(\mathbf{x})=\int_{\Omega} \Psi^{\prime}(\mathbf{x}-\mathbf{y}) \mathbf{U}(\mathbf{y}) d \mathbf{y}
$$

is a solution of (2.43).

On the other hand, by virtue of (2.8), (2.29) and (2.43) we have

$$
\mathbf{F}(\mathbf{x})=\mathbf{A}\left(\mathbf{D}_{\mathbf{x}}\right) \mathbf{U}(\mathbf{x})=\mathbf{A}\left(\mathbf{D}_{\mathbf{x}}\right) \mathbf{L}\left(\mathbf{D}_{\mathbf{x}}\right) \mathbf{W}(\mathbf{x})=\mathbf{\Lambda}(\Delta) \mathbf{W}(\mathbf{x})
$$

Hence, $\mathbf{W}$ is a solution of (2.43). $\diamond$

Remark 2.4. Quite similarly as in theorem 2.2 we can construct the fundamental matrix $\Psi^{\prime}(\mathbf{x})$ of the operator $\mathbf{L}\left(\mathbf{D}_{\mathbf{x}}\right)$ by elementary functions. 


\subsection{Green's formulas. Representations of general solutions}

Thus, on the basis of theorems 2.8 and 2.9 the completeness of Galerkin-type solution of system (2.6) is proved.

Now we consider the system of homogeneous equations (2.10). We have the following results.

Theorem 2.10. If metaharmonic function $\varphi_{j}$ and metaharmonic vector function $\boldsymbol{\psi}=\left(\psi_{1}, \psi_{2}, \psi_{3}\right)$ are solutions of equations

$$
\left(\Delta+\tau_{j}^{2}\right) \varphi_{j}(\mathbf{x})=0, \quad j=1,2
$$

and

$$
\left(\Delta+\tau_{3}^{2}\right) \boldsymbol{\psi}(\mathbf{x})=\mathbf{0}, \quad \operatorname{div} \boldsymbol{\psi}(\mathbf{x})=0,
$$

respectively, then $\mathbf{U}=(\mathbf{u}, \varphi)$ is a solution of the homogeneous equation (2.10), where

$$
\begin{aligned}
& \mathbf{u}(\mathbf{x})=\nabla\left[c_{1} \varphi_{1}(\mathbf{x})+c_{2} \varphi_{2}(\mathbf{x})\right]+\boldsymbol{\psi}(\mathbf{x}) \\
& \varphi(\mathbf{x})=\varphi_{1}(\mathbf{x})+\varphi_{2}(\mathbf{x})
\end{aligned}
$$

for $\mathbf{x} \in \Omega ; \Omega$ is an arbitrary domain in $R^{3}$ and

$$
c_{j}=\frac{1}{\rho \omega^{2} \nu_{1}}\left[\left(\alpha_{1} \tau_{j}^{2}-\xi_{2}\right) \mu_{2}-b_{1} \nu_{1}\right], \quad j=1,2 .
$$

Proof. Keeping in mind the relations (2.45)-(2.48) and

$$
\left(-\mu_{2} \tau_{j}^{2}+\rho \omega^{2}\right) c_{j}+b_{1}=0, \quad j=1,2
$$




\subsection{Green's formulas. Representations of general solutions}

we obtain by direct calculation

$$
\begin{aligned}
\mu_{1} \Delta \mathbf{u} & +\left(\lambda_{1}+\mu_{1}\right) \nabla \operatorname{div} \mathbf{u}+b_{1} \operatorname{grad} \varphi+\rho \omega^{2} \mathbf{u} \\
= & -\mu_{1} \nabla\left(c_{1} \tau_{1}^{2} \varphi_{1}+c_{2} \tau_{2}^{2} \varphi_{2}\right)-\left(\lambda_{1}+\mu_{1}\right) \nabla\left(c_{1} \tau_{1}^{2} \varphi_{1}+c_{2} \tau_{2}^{2} \varphi_{2}\right) \\
& +b_{1} \nabla\left(\varphi_{1}+\varphi_{2}\right)+\rho \omega^{2} \nabla\left(c_{1} \varphi_{1}+c_{2} \varphi_{2}\right)+\mu_{1} \Delta \boldsymbol{\psi}+\rho \omega^{2} \boldsymbol{\psi} \\
= & {\left[\left(-\mu_{2} \tau_{1}^{2}+\rho \omega^{2}\right) c_{1}+b_{1}\right] \nabla \varphi_{1}+\left[\left(-\mu_{2} \tau_{2}^{2}+\rho \omega^{2}\right) c_{2}+b_{1}\right] \nabla \varphi_{2}=\mathbf{0} . }
\end{aligned}
$$

Quite similarly, by virtue of (2.47) and

$$
\nu_{1} \tau_{j}^{2} c_{j}-\alpha_{1} \tau_{j}^{2}+\xi_{2}=0, \quad j=1,2
$$

we have

$$
\begin{gathered}
\left(\alpha_{1} \Delta+\xi_{2}\right) \varphi-\nu_{1} \operatorname{div} \mathbf{u}=-\left(\alpha_{1} \tau_{1}^{2}-\xi_{2}\right) \varphi_{1}-\left(\alpha_{1} \tau_{2}^{2}-\xi_{2}\right) \varphi_{2}+\nu_{1}\left(c_{1} \tau_{1}^{2} \varphi_{1}+c_{2} \tau_{2}^{2} \varphi_{2}\right) \\
=\left(\nu_{1} \tau_{1}^{2} c_{1}-\alpha_{1} \tau_{1}^{2}+\xi_{2}\right) \varphi_{1}+\left(\nu_{1} \tau_{2}^{2} c_{2}-\alpha_{1} \tau_{1}^{2}+\xi_{2}\right) \varphi_{2}=0 .
\end{gathered}
$$

Theorem 2.11. If $\mathbf{U}=(\mathbf{u}, \varphi)$ is a solution of the homogeneous equation (2.10) in $\Omega$, then $\mathbf{U}$ is represented by (2.47), where $\varphi_{j}$ and $\boldsymbol{\psi}=\left(\psi_{1}, \psi_{2}, \psi_{3}\right)$ are solutions of (2.45) and (2.46), respectively; $\Omega$ is an arbitrary domain in $R^{3}$ and $c_{j}(j=1,2)$ is given by (2.48).

Proof. Applying the operator div to the first equation of (2.10) from system (2.10) we have

$$
\begin{aligned}
& \left(\mu_{2} \Delta+\rho \omega^{2}\right) \operatorname{div} \mathbf{u}+b_{1} \Delta \varphi=0, \\
& \left(\alpha_{1} \Delta+\xi_{2}\right) \varphi-\nu_{1} \operatorname{div} \mathbf{u}=0 .
\end{aligned}
$$

Clearly, we obtain from (2.49)

$$
\left(\Delta+\tau_{1}^{2}\right)\left(\Delta+\tau_{2}^{2}\right) \varphi=0 .
$$




\subsection{Green's formulas. Representations of general solutions}

Now applying the operator curl to the first equation of (2.10) it follows that

$$
\left(\Delta+\tau_{3}^{2}\right) \operatorname{curl} \mathbf{u}=0
$$

We introduce the notation

$$
\begin{aligned}
& \varphi_{1}=\frac{1}{\tau_{2}^{2}-\tau_{1}^{2}}\left(\Delta+\tau_{2}^{2}\right) \varphi, \quad \varphi_{2}=\frac{1}{\tau_{1}^{2}-\tau_{2}^{2}}\left(\Delta+\tau_{1}^{2}\right) \varphi \\
& \boldsymbol{\psi}=\frac{\mu_{1}}{\rho \omega^{2}} \operatorname{curl} \operatorname{curl} \mathbf{u} .
\end{aligned}
$$

Taking into account (2.50) - (2.52), the function $\varphi_{j}$ and vector function $\boldsymbol{\psi}$ are the solutions of (2.45) and (2.46), respectively, and the function $\varphi$ is represented by $(2.47)$.

Now we prove the first relation of (2.47). Obviously, on the basis of (2.45) the second equation of (2.49) we can rewrite in the form

$$
\operatorname{div} \mathbf{u}=c_{3} \varphi_{1}+c_{4} \varphi_{2}
$$

where

$$
c_{j}=\frac{1}{\nu_{1}}\left(\xi_{2}-\alpha_{1} \tau_{j-2}^{2}\right), \quad j=3,4 .
$$

Keeping in mind (2.52), (2.53) and identity

$$
\Delta \mathbf{u}=\nabla \operatorname{div} \mathbf{u}-\operatorname{curl} \operatorname{curl} \mathbf{u}
$$

from (2.49) we obtain

$$
\begin{aligned}
\mathbf{u} & =-\frac{1}{\rho \omega^{2}} \nabla\left[\mu_{2} \operatorname{div} \mathbf{u}+b_{1} \varphi\right]+\boldsymbol{\psi} \\
& =-\frac{1}{\rho \omega^{2}} \nabla\left[\left(\mu_{2} c_{3}+b_{1}\right) \varphi_{1}+\left(\mu_{2} c_{4}+b_{1}\right) \varphi_{2}\right]+\boldsymbol{\psi} .
\end{aligned}
$$

Finally, from (2.54) we get the first relation of $(2.47)$. $\diamond$

Hence, on the basis of theorems 2.10 and 2.11 the completeness of solution of 


\subsection{Green's formulas. Representations of general solutions}

the homogeneous equation (2.10) is proved.

Remark 2.5. Contemporary treatment of the various BVPs of the theories of elasticity and thermoelasticity usually begins with the representation of a solution of field equations in terms of elementary (harmonic, biharmonic, metaharmonic and etc.) functions. In the classical theories of elasticity and thermoelasticity the Boussinesq-Somigliana-Galerkin, Boussinesq-Papkovitch-Neuber, GreenLamé, Naghdi-Hsu and Cauchy-Kovalevski-Somigliana solutions are well known (for details, see Gurtin [91], Hetnarski and Ignaczak [92]). A review of the history of these solutions is given in Wang et al. [96]. The Galerkin type solution (see Galerkin [97]) of equations of classical elastokinetics was obtained by Iacovache [99]. In the linear theory of elasticity for materials with voids, the BoussinesqPapkovitch-Neuber, Green-Lamé and Cauchy-Kovalevski-Somigliana type solutions were obtained by Chandrasekharaiah [99, 100]. The representation theorem of Galerkin type in the theory of thermoelasticity for materials with voids was proved by Ciarletta [43]. 


\section{Chapter 3}

\section{Boundary value problems in the theory of viscoelasticity for materials with voids}

\subsection{Basic boundary value problems}

The basic internal and external BVPs of steady vibration in the theory of viscoelasticity for Kelvin-Voigt materials with voids are formulated as follows.

Find a regular (classical) solution to system (2.9) for $\mathbf{x} \in \Omega^{+}$satisfying the boundary condition

$$
\lim _{\Omega^{+} \ni \mathbf{x} \rightarrow \mathbf{z} \in S} \mathbf{U}(\mathbf{x}) \equiv\{\mathbf{U}(\mathbf{z})\}^{+}=\mathbf{f}(\mathbf{z})
$$

in the Problem $(I)_{\mathbf{F}, \mathbf{f}}^{+}$, and

$$
\left\{\mathbf{P}\left(\mathbf{D}_{\mathbf{z}}, \mathbf{n}(\mathbf{z})\right) \mathbf{U}(\mathbf{z})\right\}^{+}=\mathbf{f}(\mathbf{z})
$$

in the Problem $(I I)_{\mathbf{F}, \mathbf{f}}^{+}$, where the matrix differential operator $\mathbf{P}\left(\mathbf{D}_{\mathbf{z}}, \mathbf{n}(\mathbf{z})\right)$ is defined by (2.35), $\mathbf{F}$ and $\mathbf{f}$ are the known four-component vector functions.

Find a regular (classical) solution to system (2.9) for $\mathrm{x} \in \Omega^{-}$satisfying the 
boundary condition

$$
\lim _{\Omega^{-} \ni \mathbf{x} \rightarrow \mathbf{z} \in S} \mathbf{U}(\mathbf{x}) \equiv\{\mathbf{U}(\mathbf{z})\}^{-}=\mathbf{f}(\mathbf{z})
$$

in the Problem $(I)_{\mathbf{F}, \mathbf{f}}^{-}$, and

$$
\left\{\mathbf{P}\left(\mathbf{D}_{\mathbf{z}}, \mathbf{n}(\mathbf{z})\right) \mathbf{U}(\mathbf{z})\right\}^{-}=\mathbf{f}(\mathbf{z})
$$

in the Problem $(I I)_{\mathbf{F}, \mathbf{f}}^{-}$. Here $\mathbf{F}$ and $\mathbf{f}$ are the known four-component vector functions, supp $\mathbf{F}$ is a finite domain in $\Omega^{-}$, and $\mathbf{n}(\mathbf{z})$ is the external (with respect to $\Omega^{+}$) unit normal vector to $S$ at $\mathbf{z}$.

In the Sections 3.2 and 3.4 the uniqueness and existence theorems for classical solutions of the BVPs $(K)_{\mathbf{F}, \mathbf{f}}^{+}$and $(K)_{\mathbf{F}, \mathbf{f}}^{-}$are proved by using the potential method and the theory of singular integral equations, respectively, where $K=I, I I$.

\subsection{Uniqueness theorems}

In the sequel we use the matrix differential operators

$$
\mathbf{B}\left(\mathbf{D}_{\mathbf{x}}\right)=\left(B_{l j}\left(\mathbf{D}_{\mathbf{x}}\right)\right)_{3 \times 3}, \quad \mathbf{T}\left(\mathbf{D}_{\mathbf{x}}, \mathbf{n}\right)=\left(T_{l j}\left(\mathbf{D}_{\mathbf{x}}, \mathbf{n}\right)\right)_{3 \times 3},
$$

where

$$
\begin{aligned}
& B_{l j}\left(\mathbf{D}_{\mathbf{x}}\right)=\mu_{1} \Delta \delta_{l j}+\left(\lambda_{1}+\mu_{1}\right) \frac{\partial^{2}}{\partial x_{l} \partial x_{j}}, \\
& T_{l j}\left(\mathbf{D}_{\mathbf{x}}, \mathbf{n}\right)=\mu_{1} \delta_{l j} \frac{\partial}{\partial \mathbf{n}}+\mu_{1} n_{j} \frac{\partial}{\partial x_{l}}+\lambda_{1} n_{l} \frac{\partial}{\partial x_{j}}, \quad l, j=1,2,3 .
\end{aligned}
$$

Obviously, $\mathbf{T}\left(\mathbf{D}_{\mathbf{x}}, \mathbf{n}\right)$ is the stress operator in the classical theory of elasticity (see Kupradze et al. [83]). 
Theorem 3.1. If conditions

$$
\begin{aligned}
& \mu^{*}>0, \quad 3 \lambda^{*}+2 \mu^{*}>0, \quad \alpha^{*}>0 \\
& \left(3 \lambda^{*}+2 \mu^{*}\right) \xi^{*}>\frac{3}{4}\left(b^{*}+\nu^{*}\right)^{2}
\end{aligned}
$$

are satisfied, then the internal $B V P(K)_{\mathbf{F}, \mathbf{f}}^{+}$admits at most one regular solution, where $K=I, I I$.

Proof. Suppose that there are two regular solutions of problem $(K)_{\mathbf{F}, \mathbf{f}}^{+}$. Then their difference $\mathbf{U}$ corresponds to zero data $(\mathbf{F}=\mathbf{f}=\mathbf{0})$, i.e. $\mathbf{U}$ is a regular solution of problem $(K)_{\mathbf{0}, \mathbf{0}}^{+}$.

On account of (2.10) from Green's formulas of the classical theory of elasticity (see Kupradze et al. [83])

$$
\begin{aligned}
& \int_{\Omega^{+}}\left[\mathbf{B}\left(\mathbf{D}_{\mathbf{x}}\right) \mathbf{u} \cdot \mathbf{u}+W\left(\mathbf{u}, \lambda_{1}, \mu_{1}\right)\right] d \mathbf{x}=\int_{S} \mathbf{T u} \cdot \mathbf{u} d_{\mathbf{z}} S \\
& \int_{\Omega^{+}}\left[\Delta \varphi \bar{\varphi}+|\nabla \varphi|^{2}\right] d \mathbf{x}=\int_{S} \frac{\partial \varphi}{\partial \mathbf{n}} \bar{\varphi} d_{\mathbf{z}} S
\end{aligned}
$$

and identity

$$
\int_{\Omega^{+}}(\nabla \varphi \cdot \mathbf{u}+\varphi \operatorname{div} \overline{\mathbf{u}}) d \mathbf{x}=\int_{S} \varphi \mathbf{n} \cdot \mathbf{u} d_{\mathbf{z}} S
$$

it follows that

$$
\begin{aligned}
& \int_{\Omega^{+}}\left[W\left(\mathbf{u}, \lambda_{1}, \mu_{1}\right)-\rho \omega^{2}|\mathbf{u}|^{2}+b_{1} \varphi \operatorname{div} \overline{\mathbf{u}}\right] d \mathbf{x}=\int_{S}\left(\mathbf{T u}+b_{1} \varphi \mathbf{n}\right) \cdot \mathbf{u} d_{\mathbf{z}} S, \\
& \int_{\Omega^{+}}\left[\alpha_{1}|\nabla \varphi|^{2}-\xi_{2}|\varphi|^{2}+\nu_{1} \operatorname{div} \mathbf{u} \bar{\varphi}\right] d \mathbf{x}=\alpha_{1} \int_{S} \frac{\partial \varphi}{\partial \mathbf{\varphi}} \bar{\varphi} d_{\mathbf{z}} S,
\end{aligned}
$$


where

$$
\begin{aligned}
& W\left(\mathbf{u}, \lambda_{1}, \mu_{1}\right)=\frac{1}{3}\left(3 \lambda_{1}+2 \mu_{1}\right)|\operatorname{div} \mathbf{u}|^{2} \\
& +\mu_{1}\left[\frac{1}{2} \sum_{l, j=1 ; l \neq j}^{3}\left|\frac{\partial u_{j}}{\partial x_{l}}+\frac{\partial u_{l}}{\partial x_{j}}\right|^{2}+\frac{1}{3} \sum_{l, j=1}^{3}\left|\frac{\partial u_{l}}{\partial x_{l}}-\frac{\partial u_{j}}{\partial x_{j}}\right|^{2}\right] .
\end{aligned}
$$

Clearly, $W\left(\mathbf{u}, \lambda_{1}, \mu_{1}\right)=W(\mathbf{u}, \lambda, \mu)-i \omega W\left(\mathbf{u}, \lambda^{*}, \mu^{*}\right)$. In view of (3.6) we get

$$
\begin{aligned}
& \int_{\Omega^{+}}\left[W\left(\mathbf{u}, \lambda_{1}, \mu_{1}\right)-\rho \omega^{2}|\mathbf{u}|^{2}+\alpha_{1}|\nabla \varphi|^{2}-\xi_{2}|\varphi|^{2}+\left(b_{1} \varphi \operatorname{div} \overline{\mathbf{u}}+\nu_{1} \operatorname{div} \mathbf{u} \bar{\varphi}\right)\right] d \mathbf{x} \\
& =\int_{S}\left[\left(\mathbf{T u}+b_{1} \varphi \mathbf{n}\right) \cdot \mathbf{u}+\alpha_{1} \frac{\partial \varphi}{\partial \mathbf{n}} \bar{\varphi}\right] d_{\mathbf{z}} S
\end{aligned}
$$

and on the basis of homogeneous boundary condition and the identity

$$
\operatorname{Im}\left(b_{1} \varphi \operatorname{div} \overline{\mathbf{u}}+\nu_{1} \operatorname{div} \mathbf{u} \bar{\varphi}\right)=-\omega\left(b^{*}+\nu^{*}\right) \operatorname{Re}(\varphi \operatorname{div} \overline{\mathbf{u}})
$$

we obtain

$$
\int_{\Omega^{+}}\left[W\left(\mathbf{u}, \lambda^{*}, \mu^{*}\right)-\left(b^{*}+\nu^{*}\right) \operatorname{Re}(\varphi \operatorname{div} \overline{\mathbf{u}})+\xi^{*}|\varphi|^{2}+\alpha^{*}|\nabla \varphi|^{2}\right] d \mathbf{x}=0 .
$$

Obviously, with the help of (3.5) it follows that

$$
\frac{1}{3}\left(3 \lambda^{*}+2 \mu^{*}\right)|\operatorname{div} \mathbf{u}|^{2}-\left(b^{*}+\nu^{*}\right) \operatorname{Re}(\varphi \operatorname{div} \overline{\mathbf{u}})+\xi^{*}|\varphi|^{2} \geq 0
$$

and from (3.8) we have

$$
W\left(\mathbf{u}, \lambda^{*}, \mu^{*}\right)-\left(b^{*}+\nu^{*}\right) \operatorname{Re}(\varphi \operatorname{div} \overline{\mathbf{u}})+\xi^{*}|\varphi|^{2}+\alpha^{*}|\operatorname{grad} \varphi|^{2}=0 .
$$

It is easy to verify that on the basis of (3.5) the last equation leads to the following 
relations

$$
\begin{aligned}
& \varphi(\mathbf{x})=0, \quad \operatorname{div} \mathbf{u}(\mathbf{x})=0, \quad \frac{\partial u_{j}}{\partial x_{l}}+\frac{\partial u_{l}}{\partial x_{j}}=0, \\
& \frac{\partial u_{l}}{\partial x_{l}}-\frac{\partial u_{j}}{\partial x_{j}}=0, \quad l, j=1,2,3
\end{aligned}
$$

for $\mathbf{x} \in \Omega^{+}$. In view of (3.9) we get $W(\mathbf{u}, \lambda, \mu)=0$ and $W\left(\mathbf{u}, \lambda^{*}, \mu^{*}\right)=0$. Hence, $W\left(\mathbf{u}, \lambda_{1}, \mu_{1}\right)=0$. Finally, from the first equation of (3.6) we obtain $\mathbf{u}(\mathbf{x})=\mathbf{0}$. Thus, $\mathbf{U}(\mathbf{x})=\mathbf{0}$ for $\mathbf{x} \in \Omega^{+}$.

Lemma 3.1. If $\mathbf{U}=(\mathbf{u}, \varphi) \in C^{2}(\Omega)$ is a solution of the system (2.10) for $\mathbf{x} \in \Omega$, then

$$
\mathbf{u}(\mathbf{x})=\sum_{j=1}^{3} \mathbf{u}^{(j)}(\mathbf{x}), \quad \varphi(\mathbf{x})=\sum_{l=1}^{2} \varphi^{(l)}(\mathbf{x}),
$$

where $\Omega$ is an arbitrary domain in $R^{3}, \mathbf{u}^{(j)}$ and $\varphi^{(l)}$ satisfy the following equations

$$
\begin{aligned}
& \left(\Delta+\tau_{j}^{2}\right) \mathbf{u}^{(j)}(\mathbf{x})=\mathbf{0}, \quad\left(\Delta+\tau_{l}^{2}\right) \varphi^{(l)}(\mathbf{x})=0 \\
& l=1,2, \quad j=1,2,3 .
\end{aligned}
$$

Proof. Applying the operator div to the first equation of (2.10) we get

$$
\begin{aligned}
& \left(\mu_{2} \Delta+\rho \omega^{2}\right) \operatorname{div} \mathbf{u}+b_{1} \Delta \varphi=0, \\
& \left(\alpha_{1} \Delta+\xi_{2}\right) \varphi-\nu_{1} \operatorname{div} \mathbf{u}=0 .
\end{aligned}
$$

Clearly, from system (3.12) we have

$$
\begin{aligned}
& \left(\Delta+\tau_{1}^{2}\right)\left(\Delta+\tau_{2}^{2}\right) \operatorname{div} \mathbf{u}=0, \\
& \left(\Delta+\tau_{1}^{2}\right)\left(\Delta+\tau_{2}^{2}\right) \varphi=0
\end{aligned}
$$

Now, applying the operator $\left(\Delta+\tau_{1}^{2}\right)\left(\Delta+\tau_{2}^{2}\right)$ to the first equation of $(2.10)$ and using (3.13) we obtain

$$
\left(\Delta+\tau_{1}^{2}\right)\left(\Delta+\tau_{2}^{2}\right)\left(\Delta+\tau_{3}^{2}\right) \mathbf{u}=\mathbf{0} .
$$


We introduce the notation

$$
\begin{array}{ll}
\mathbf{u}^{(j)} & =\prod_{p=1 ; p \neq j}^{3}\left(\tau_{p}^{2}-\tau_{j}^{2}\right)^{-1}\left(\Delta+\tau_{p}^{2}\right) \mathbf{u}, \quad j=1,2,3, \\
\varphi^{(l)} & =\prod_{p=1 ; p \neq l}^{2}\left(\tau_{p}^{2}-\tau_{l}^{2}\right)^{-1}\left(\Delta+\tau_{p}^{2}\right) \varphi, \quad l=1,2 .
\end{array}
$$

By virtue of (3.13) and (3.14) the relations (3.10) and (3.11) can be easily obtained from (3.15).

Now let us establish the uniqueness of a regular solution of external BVPs.

Theorem 3.2. If conditions (3.5) are satisfied, then the external $B V P(K)_{\mathbf{F}, \mathbf{f}}^{-}$ admits at most one regular solution, where $K=I, I I$.

Proof. Suppose that there are two regular solutions of problem $(K)_{\mathbf{F}, \mathbf{f}}^{-}$. Then their difference $\mathbf{U}=\left(U_{1}, U_{2}, U_{3}, U_{4}\right)$ corresponds to zero data $(\mathbf{F}=\mathbf{f}=\mathbf{0})$, i.e. $\mathbf{U}$ is a regular solution of the problem $(K)_{\mathbf{0}, \mathbf{0}}^{-}$.

Let $\Omega_{r}$ be a sphere of sufficiently large radius $r$ so that $\bar{\Omega}^{+} \subset \Omega_{r}$. By virtue of homogeneous boundary condition $(\mathbf{f}=\mathbf{0})$, the formula $(2.36)$ for the domain $\Omega_{r}^{-}=\Omega^{-} \cap \Omega_{r}$ can be rewritten as

$$
\begin{aligned}
& \int_{\Omega_{r}^{-}}\left[W\left(\mathbf{u}, \lambda_{1}, \mu_{1}\right)-\rho \omega^{2}|\mathbf{u}|^{2}+\alpha_{1}|\nabla \varphi|^{2}-\xi_{2}|\varphi|^{2}+\left(b_{1} \varphi \operatorname{div} \overline{\mathbf{u}}+\nu_{1} \operatorname{div} \mathbf{u} \bar{\varphi}\right)\right] d \mathbf{x} \\
& \quad=\int_{S_{r}}\left[\left(\mathbf{T u}+b_{1} \varphi \mathbf{n}\right) \cdot \mathbf{u}+\alpha_{1} \frac{\partial \varphi}{\partial \mathbf{n}} \bar{\varphi}\right] d_{\mathbf{z}} S
\end{aligned}
$$

where $S_{r}$ is the boundary of the sphere $\Omega_{r}$. From (3.16) we have

$$
N=\lim _{r \rightarrow \infty} \int_{S_{r}}\left[\left(\mathbf{T u}+b_{1} \varphi \mathbf{n}\right) \cdot \mathbf{u}+\alpha_{1} \frac{\partial \varphi}{\partial \mathbf{n}} \bar{\varphi}\right] d_{\mathbf{z}} S
$$


where

$$
N=\int_{\Omega^{-}}\left[W\left(\mathbf{u}, \lambda^{*}, \mu^{*}\right)-\left(b^{*}+\nu^{*}\right) \operatorname{Re}(\varphi \operatorname{div} \overline{\mathbf{u}})+\xi^{*}|\varphi|^{2}+\alpha^{*}|\nabla \varphi|^{2}\right] d \mathbf{x} .
$$

Obviously, by condition (3.5) it follows from (3.18) that $N \geq 0$.

On the other hand, keeping in mind the conditions (1.1), (2.34) and (3.10) from (2.40) we obtain

$$
\begin{aligned}
& U_{j}(x)=e^{-\tau_{0}|\mathbf{x}|} O\left(|\mathbf{x}|^{-1}\right), \\
& U_{j, l}(x)=e^{-\tau_{0}|\mathbf{x}|} O\left(|\mathbf{x}|^{-1}\right) \quad \text { for } \quad|\mathbf{x}| \gg 1,
\end{aligned}
$$

where $\tau_{0}=\min \left\{\operatorname{Im} \tau_{1}, \operatorname{Im} \tau_{2}, \operatorname{Im} \tau_{3}\right\}>0, l=1,2,3, j=1,2,3,4$. On account of (3.19) from (3.17) it follows that $N=0$. Hence, from (3.18) we get

$$
\int_{\Omega^{-}}\left[W\left(\mathbf{u}, \lambda^{*}, \mu^{*}\right)-\left(b^{*}+\nu^{*}\right) \operatorname{Re}(\varphi \operatorname{div} \overline{\mathbf{u}})+\xi^{*}|\varphi|^{2}+\alpha^{*}|\operatorname{grad} \varphi|^{2}\right] d \mathbf{x}=0 .
$$

Quite similarly as in theorem 3.1 on the basis of (3.5) from (3.20) we obtain $\mathbf{U}(\mathbf{x})=\mathbf{0}$ for $\mathbf{x} \in \Omega^{-}$. $\diamond$

Remark 3.1. The uniqueness theorems in the linear theory of elasticity for materials with voids are proved by Chandrasekharaiah [34]. Basic results as well as an extensive review on uniqueness theorems in the classical theory of elasticity can be found in the books by Knops and Payne [101], Kupradze et al. [83].

\subsection{Basic properties of elastopotentials}

On the basis of Somigliana-type integral representation of regular vector (see, (2.39)) we introduce the following notations

$$
\mathbf{Z}^{(1)}(\mathbf{x}, \mathbf{g})=\int_{S} \boldsymbol{\Gamma}(\mathbf{x}-\mathbf{y}) \mathbf{g}(\mathbf{y}) d_{\mathbf{y}} S
$$




$$
\begin{aligned}
& \mathbf{Z}^{(2)}(\mathbf{x}, \mathbf{g})=\int_{S}\left[\tilde{\mathbf{P}}\left(\mathbf{D}_{\mathbf{y}}, \mathbf{n}(\mathbf{y})\right) \boldsymbol{\Gamma}^{\top}(\mathbf{x}-\mathbf{y})\right]^{\top} \mathbf{g}(\mathbf{y}) d_{\mathbf{y}} S \\
& \mathbf{Z}^{(3)}\left(\mathbf{x}, \boldsymbol{\phi}, \Omega^{ \pm}\right)=\int_{\Omega^{ \pm}} \boldsymbol{\Gamma}(\mathbf{x}-\mathbf{y}) \boldsymbol{\phi}(\mathbf{y}) d \mathbf{y}
\end{aligned}
$$

where $\mathbf{g}$ and $\phi$ are four-component vectors.

As in classical theory of elasticity (see, e.g. Kupradze et al. [83]), the vector functions $\mathbf{Z}^{(1)}(\mathbf{x}, \mathbf{g}), \mathbf{Z}^{(2)}(\mathbf{x}, \mathbf{g})$ and $\mathbf{Z}^{(3)}\left(\mathbf{x}, \boldsymbol{\phi}, \Omega^{ \pm}\right)$are called single-layer, doublelayer and volume potentials in the linear theory of viscoelasticity for Kelvin-Voigt materials with voids, respectively.

Obviously, on the basis of (2.39) and (2.40), the regular vector in $\Omega^{+}$and $\Omega^{-}$ is represented by sum of the elastopotentials as follows

$$
\mathbf{U}(\mathbf{x})=\mathbf{Z}^{(2)}\left(\mathbf{x},\{\mathbf{U}\}^{+}\right)-\mathbf{Z}^{(1)}\left(\mathbf{x},\{\mathbf{P} \mathbf{U}\}^{+}\right)+\mathbf{Z}^{(3)}\left(\mathbf{x}, \mathbf{A} \mathbf{U}, \Omega^{+}\right) \quad \text { for } \quad \mathbf{x} \in \Omega^{+}
$$

and

$$
\mathbf{U}(\mathbf{x})=-\mathbf{Z}^{(2)}\left(\mathbf{x},\{\mathbf{U}\}^{-}\right)+\mathbf{Z}^{(1)}\left(\mathbf{x},\{\mathbf{P} \mathbf{U}\}^{-}\right)+\mathbf{Z}^{(3)}\left(\mathbf{x}, \mathbf{A} \mathbf{U}, \Omega^{-}\right) \quad \text { for } \quad \mathbf{x} \in \Omega^{-}
$$

respectively.

First we establish the basic properties of elastopotentials.

Theorem 3.3. If $S \in C^{j+1, p}, \mathrm{~g} \in C^{j, p^{\prime}}(S), 0<p^{\prime}<p \leq 1$, and $j$ is a nonnegative integer, then:

a)

$$
\mathbf{Z}^{(1)}(\cdot, \mathbf{g}) \in C^{0, p^{\prime}}\left(\mathbb{R}^{3}\right) \cap C^{j+1, p^{\prime}}\left(\bar{\Omega}^{ \pm}\right) \cap C^{\infty}\left(\Omega^{ \pm}\right),
$$

b)

$$
\mathbf{A}\left(\mathbf{D}_{\mathbf{x}}\right) \mathbf{Z}^{(1)}(\mathbf{x}, \mathbf{g})=\mathbf{0}, \quad \mathbf{x} \in \Omega^{ \pm},
$$

c)

$$
\left\{\mathbf{P}\left(\mathbf{D}_{\mathbf{z}}, \mathbf{n}(\mathbf{z})\right) \mathbf{Z}^{(1)}(\mathbf{z}, \mathbf{g})\right\}^{ \pm}=\mp \frac{1}{2} \mathbf{g}(\mathbf{z})+\mathbf{P}\left(\mathbf{D}_{\mathbf{z}}, \mathbf{n}(\mathbf{z})\right) \mathbf{Z}^{(1)}(\mathbf{z}, \mathbf{g}), \quad \mathbf{z} \in S,
$$


d)

$$
\mathbf{P}\left(\mathbf{D}_{\mathbf{z}}, \mathbf{n}(\mathbf{z})\right) \mathbf{Z}^{(1)}(\mathbf{z}, \mathbf{g})
$$

is a singular integral for $\mathbf{z} \in S$.

Theorem 3.4. If $S \in C^{j+1, p}, \mathbf{g} \in C^{j, p^{\prime}}(S), 0<p^{\prime}<p \leq 1$, then:

a)

$$
\mathbf{Z}^{(2)}(\cdot, \mathbf{g}) \in C^{j, p^{\prime}}\left(\bar{\Omega}^{ \pm}\right) \cap C^{\infty}\left(\Omega^{ \pm}\right),
$$

b)

$$
\mathbf{A}\left(\mathbf{D}_{\mathbf{x}}\right) \mathbf{Z}^{(2)}(\mathbf{x}, \mathbf{g})=\mathbf{0}, \quad \mathbf{x} \in \Omega^{ \pm},
$$

c)

$$
\left\{\mathbf{Z}^{(2)}(\mathbf{z}, \mathbf{g})\right\}^{ \pm}= \pm \frac{1}{2} \mathbf{g}(\mathbf{z})+\mathbf{Z}^{(2)}(\mathbf{z}, \mathbf{g}), \quad \mathbf{z} \in S
$$

for the non-negative integer $j$,

d)

$$
\mathbf{Z}^{(2)}(\mathbf{z}, \mathbf{g})
$$

is a singular integral for $\mathbf{z} \in S$,

e)

$$
\left\{\mathbf{P}\left(\mathbf{D}_{\mathbf{z}}, \mathbf{n}(\mathbf{z})\right) \mathbf{Z}^{(2)}(\mathbf{z}, \mathbf{g})\right\}^{+}=\left\{\mathbf{P}\left(\mathbf{D}_{\mathbf{z}}, \mathbf{n}(\mathbf{z})\right) \mathbf{Z}^{(2)}(\mathbf{z}, \mathbf{g})\right\}^{-},
$$

for the natural number $j$ and $\mathbf{z} \in S$.

Theorem 3.5. If $S \in C^{1, p}, \phi \in C^{0, p^{\prime}}\left(\Omega^{+}\right), 0<p^{\prime}<p \leq 1$, then:

a)

$$
\mathbf{Z}^{(3)}\left(\cdot, \phi, \Omega^{+}\right) \in C^{1, p^{\prime}}\left(\mathbb{R}^{3}\right) \cap C^{2}\left(\Omega^{+}\right) \cap C^{2, p^{\prime}}\left(\bar{\Omega}_{0}^{+}\right),
$$

b)

$$
\mathbf{A}\left(\mathbf{D}_{\mathbf{x}}\right) \mathbf{Z}^{(3)}\left(x, \phi, \Omega^{+}\right)=\phi(\mathbf{x}), \quad \mathbf{x} \in \Omega^{+},
$$

where $\Omega_{0}^{+}$is a domain in $\mathbb{R}^{3}$ and $\Omega_{0}^{+} \subset \Omega^{+}$.

Theorem 3.6. If $S \in C^{1, p}, \operatorname{supp} \phi=\Omega \subset \Omega^{-}, \quad \phi \in C^{0, p^{\prime}}\left(\Omega^{-}\right), 0<p^{\prime}<p \leq 1$, then:

a)

$$
\mathbf{Z}^{(3)}\left(\cdot, \phi, \Omega^{-}\right) \in C^{1, p^{\prime}}\left(\mathbb{R}^{3}\right) \cap C^{2}\left(\Omega^{-}\right) \cap C^{2, p^{\prime}}\left(\bar{\Omega}_{0}^{-}\right),
$$


b)

$$
\mathbf{A}\left(\mathbf{D}_{\mathbf{x}}\right) \mathbf{Z}^{(3)}\left(\mathbf{x}, \phi, \Omega^{-}\right)=\phi(\mathbf{x}), \quad \mathbf{x} \in \Omega^{-}
$$

where $\Omega$ is a finite domain in $\mathbb{R}^{3}$ and $\bar{\Omega}_{0}^{-} \subset \Omega^{-}$.

Theorems 3.3 to 3.6 can be proved similarly to the corresponding theorems in the classical theory of thermoelasticity (for details, see Kupradze et al. [83]).

We introduce the notations

$$
\begin{aligned}
& \mathcal{K}^{(1)} \mathbf{g}(\mathbf{z}) \equiv \frac{1}{2} \mathbf{g}(\mathbf{z})+\mathbf{Z}^{(2)}(\mathbf{z}, \mathbf{g}) \\
& \mathcal{K}^{(2)} \mathbf{g}(\mathbf{z}) \equiv-\frac{1}{2} \mathbf{g}(\mathbf{z})+\mathbf{P}\left(\mathbf{D}_{\mathbf{z}}, \mathbf{n}(\mathbf{z})\right) \mathbf{Z}^{(1)}(\mathbf{z}, \mathbf{g}), \\
& \mathcal{K}^{(3)} \mathbf{g}(\mathbf{z}) \equiv-\frac{1}{2} \mathbf{g}(\mathbf{z})+\mathbf{Z}^{(2)}(\mathbf{z}, \mathbf{g}), \\
& \mathcal{K}^{(4)} \mathbf{g}(\mathbf{z}) \equiv \frac{1}{2} \mathbf{g}(\mathbf{z})+\mathbf{P}\left(\mathbf{D}_{\mathbf{z}}, \mathbf{n}(\mathbf{z})\right) \mathbf{Z}^{(1)}(\mathbf{z}, \mathbf{g}), \\
& \mathcal{K}_{\zeta} \mathbf{g}(\mathbf{z}) \equiv-\frac{1}{2} \mathbf{g}(\mathbf{z})+\zeta \mathbf{Z}^{(2)}(\mathbf{z}, \mathbf{g}) \quad \text { for } \quad \mathbf{z} \in S,
\end{aligned}
$$

where $\zeta$ is a complex parameter. On the basis of theorems 3.3 and $3.4, \mathcal{K}^{(j)}(j=$ $1,2,3,4)$ and $\mathcal{K}_{\zeta}$ are singular integral operators (for the definition a singular integral operator see, e.g. Mikhlin [81]).

In the sequel we need the following Lemmas.

Lemma 3.2. If conditions (3.5) are satisfied, then the singular integral operator $\mathcal{K}^{(j)}(j=1,2,3,4)$ is of the normal type.

Proof: Let $\boldsymbol{\sigma}^{(j)}=\left(\sigma_{l m}^{(j)}\right)_{4 \times 4}$ be the symbol of the singular integral operator $\mathcal{K}^{(j)}(j=1,2,3.4)$ (see, e.g. Mikhlin [81]). Taking into account (3.23) we find (for details, see, Kupradze et al. [83], Ch. IV)

$$
\operatorname{det} \boldsymbol{\sigma}^{(j)}=\left(-\frac{1}{2}\right)^{4}\left[1-\frac{\mu_{1}^{2}}{\left(\lambda_{1}+2 \mu_{1}\right)^{2}}\right]=\frac{\left(\lambda_{1}+\mu_{1}\right)\left(\lambda_{1}+3 \mu_{1}\right)}{16\left(\lambda_{1}+2 \mu_{1}\right)^{2}}
$$


Keeping in mind the relations (3.5) from (3.24) we have

$$
\operatorname{det} \boldsymbol{\sigma}^{(j)} \neq 0
$$

Hence, the operator $\mathcal{K}^{(j)}$ is of the normal type, where $j=1,2,3,4$. $\diamond$

Lemma 3.3. If $\mathcal{L}$ is a continuous curve on the complex plane connecting the origin with the point $\zeta_{0}$ and $\mathcal{K}_{\zeta}$ is a normal type operator for any $\zeta \in \mathcal{L}$, then the index of the operator $\mathcal{K}_{\zeta_{0}}$ vanishes, i.e.

$$
\text { ind } \mathcal{K}_{\zeta_{0}}=0
$$

Lemma 3.3 for the singular integral operators is proved in Kupradze et al. [83], Ch. IV.

Lemma 3.4. If conditions (3.5) are satisfied, then the Fredholm's theorems are valid for the singular integral operator $\mathcal{K}^{(j)}\left(\mathcal{K}^{(j)}\right.$ is Fredholmian), where $j=1,2,3,4$.

Proof: Let $\boldsymbol{\sigma}_{\zeta}$ and ind $\mathcal{K}_{\zeta}$ be the symbol and the index of the operator $\mathcal{K}_{\zeta}$, respectively. It may be easily shown that

$$
\operatorname{det} \boldsymbol{\sigma}_{\zeta}=\frac{\left(\lambda_{1}+2 \mu_{1}\right)^{2}-\mu_{1}^{2} \zeta^{2}}{16\left(\lambda_{1}+2 \mu_{1}\right)^{2}}
$$

and $\operatorname{det} \boldsymbol{\sigma}_{\zeta}$ vanishes only at two points $\zeta_{1}$ and $\zeta_{2}$ of the complex plane. By virtue of (3.25) and $\operatorname{det} \boldsymbol{\sigma}_{1}=\operatorname{det} \boldsymbol{\sigma}^{(1)}$ we get $\zeta_{j} \neq 1$ for $j=1,2$. By Lemma 3.3 we obtain

$$
\text { ind } \mathcal{K}^{(1)}=\text { ind } \mathcal{K}_{1}=0 \text {. }
$$

Equation ind $\mathcal{K}^{(2)}=0$ is proved in a quite similar manner. Obviously, the operators $\mathcal{K}^{(3)}$ and $\mathcal{K}^{(4)}$ are the adjoint operators for $\mathcal{K}^{(2)}$ and $\mathcal{K}^{(1)}$, respectively. Evidently,

$$
\text { ind } \mathcal{K}^{(3)}=- \text { ind } \mathcal{K}^{(2)}=0, \quad \text { ind } \mathcal{K}^{(4)}=- \text { ind } \mathcal{K}^{(1)}=0 .
$$


Thus, the singular integral operator $\mathcal{K}^{(j)}(j=1,2,3,4)$ is of the normal type with an index equal to zero. Consequently, the Fredholm's theorems are valid for $\mathcal{K}^{(j)}$ (for details, see, e.g. Mikhlin [81]).

Remark 3.2. The definitions of a normal type singular integral operator, the symbol and the index of operator are given in Mikhlin [81] and Kupradze et al. [83].

\subsection{Existence theorems}

Obviously, by theorems 3.5 and 3.6 the volume potential $\mathbf{Z}^{(3)}\left(\mathbf{x}, \mathbf{F}, \Omega^{ \pm}\right)$is a regular solution of (2.9), where $\mathbf{F} \in C^{0, p^{\prime}}\left(\Omega^{ \pm}\right), 0<p^{\prime} \leq 1$; $\operatorname{supp} \mathbf{F}$ is a finite domain in $\Omega^{-}$. Therefore, further we will consider problem $(K)_{\mathbf{0}, \mathbf{f}}^{ \pm}$for $K=I, I I$. In addition, we assume that the conditions (3.5) are satisfied.

Now we prove the existence theorems of a regular (classical) solution of problems $(K)_{\mathbf{0}, \mathbf{f}}^{-}$and $(K)_{\mathbf{0}, \mathbf{f}}^{-}$for $K=I, I I$.

Problem $(I)_{\mathbf{0}, \mathbf{f}}^{+}$. We seek a regular solution to problem $(I)_{\mathbf{0}, \mathbf{f}}^{+}$in the form

$$
\mathbf{U}(\mathbf{x})=\mathbf{Z}^{(2)}(\mathbf{x}, \mathbf{g}) \quad \text { for } \quad \mathbf{x} \in \Omega^{+},
$$

where $\mathbf{g}$ is the required four-component vector function.

By theorem 3.4 the vector function $\mathbf{U}$ is a solution of $(2.11)$ for $\mathbf{x} \in \Omega^{+}$. Keeping in mind the boundary condition (3.1) and using (3.22), from (3.26) we obtain, for determining the unknown vector $\mathbf{g}$, a singular integral equation

$$
\mathcal{K}^{(1)} \mathbf{g}(\mathbf{z})=\mathbf{f}(\mathbf{z}) \quad \text { for } \quad \mathbf{z} \in S
$$

By lemma 3.4 the Fredholm's theorems are valid for operator $\mathcal{K}^{(1)}$. We prove that (3.27) is always solvable for an arbitrary vector $\mathbf{f}$. Let us consider the associate homogeneous equation

$$
\mathcal{K}^{(4)} \mathbf{h}_{0}(\mathbf{z})=\mathbf{0} \quad \text { for } \quad \mathbf{z} \in S \text {, }
$$


where $\mathbf{h}_{0}$ is the required four-component vector function. Now we prove that (3.28) has only the trivial solution.

Indeed, let $\mathbf{h}_{0}$ be a solution of the homogeneous equation (3.28). On the basis of theorem 3.3 and (3.28) the vector function $\mathbf{V}(\mathbf{x})=\mathbf{Z}^{(1)}\left(\mathbf{x}, \mathbf{h}_{0}\right)$ is a regular solution of problem $(I I)_{\mathbf{0}, \mathbf{0}}^{-}$. Using theorem 3.2 , the problem $(I I)_{\mathbf{0}, \mathbf{0}}^{-}$has only the trivial solution, that is

$$
\mathbf{V}(\mathbf{x})=\mathbf{0} \quad \text { for } \quad \mathbf{x} \in \Omega^{-}
$$

On other hand, by theorem 3.3 and (3.29) we get

$$
\{\mathbf{V}(\mathbf{z})\}^{+}=\{\mathbf{V}(\mathbf{z})\}^{-}=\mathbf{0} \quad \text { for } \quad \mathbf{z} \in S
$$

i.e., the vector $\mathbf{V}(\mathbf{x})$ is a regular solution of problem $(I)_{\mathbf{0}, \mathbf{0}}^{+}$. Using theorem 3.1, the problem $(I)_{\mathbf{0}, \mathbf{0}}^{+}$has only the trivial solution, that is

$$
\mathbf{V}(\mathbf{x})=\mathbf{0} \quad \text { for } \quad \mathbf{x} \in \Omega^{+}
$$

By virtue of (3.29), (3.30) and identity (3.21) we obtain

$$
\mathbf{h}_{0}(\mathbf{z})=\left\{\mathbf{P}\left(\mathbf{D}_{\mathbf{z}}, \mathbf{n}\right) \mathbf{V}(\mathbf{z})\right\}^{-}-\left\{\mathbf{P}\left(\mathbf{D}_{\mathbf{z}}, \mathbf{n}\right) \mathbf{V}(\mathbf{z})\right\}^{+}=\mathbf{0} \quad \text { for } \quad \mathbf{z} \in S
$$

Thus, the homogeneous equation (3.28) has only the trivial solution and therefore (3.27) is always solvable for an arbitrary vector $\mathbf{f}$.

We have thereby proved

Theorem 3.7. If $S \in C^{2, p}$, $\mathbf{f} \in C^{1, p^{\prime}}(S), 0<p^{\prime}<p \leq 1$, then a regular solution of problem $(I)_{\mathbf{0}, \mathbf{f}}^{+}$exists, is unique and is represented by double-layer potential (3.26), where $\mathbf{g}$ is a solution of the singular integral equation (3.27) which is always solvable for an arbitrary vector $\mathbf{f}$.

Problem $(I I)_{\mathbf{0}, \mathbf{f}}^{-}$. We seek a regular solution to problem $(I I)_{\mathbf{0}, \mathbf{f}}^{-}$in the form

$$
\mathbf{U}(\mathbf{x})=\mathbf{Z}^{(1)}(\mathbf{x}, \mathbf{h}) \quad \text { for } \quad \mathbf{x} \in \Omega^{-},
$$


where $\mathbf{h}$ is the required four-component vector function.

Obviously, by theorem 3.3 the vector function $\mathbf{U}$ is a solution of (2.11) for $\mathbf{x} \in$ $\Omega^{-}$. Keeping in mind the boundary condition (3.4) and using (3.21), from (3.31) we obtain, for determining the unknown vector $\mathbf{h}$, a singular integral equation

$$
\mathcal{K}^{(4)} \mathbf{h}(\mathbf{z})=\mathbf{f}(\mathbf{z}) \quad \text { for } \quad \mathbf{z} \in S
$$

It has been proved above that the corresponding homogeneous equation (3.28) has only the trivial solution. Hence, it follows that (3.32) is always solvable.

We have thereby proved

Theorem 3.8. If $S \in C^{2, p}$, $\mathbf{f} \in C^{0, p^{\prime}}(S), 0<p^{\prime}<p \leq 1$, then a regular solution of problem $(I I)_{\mathbf{0}, \mathbf{f}}^{-}$exists, is unique and is represented by single-layer potential (3.31), where $\mathbf{h}$ is a solution of the singular integral equation (3.32) which is always solvable for an arbitrary vector $\mathbf{f}$.

Problem $(I I)_{\mathbf{0}, \mathbf{f}}^{+}$. We seek a regular solution to problem $(I I)_{\mathbf{0}, \mathbf{f}}^{+}$in the form

$$
\mathbf{U}(\mathbf{x})=\mathbf{Z}^{(1)}(\mathbf{x}, \mathbf{g}) \quad \text { for } \quad \mathbf{x} \in \Omega^{+},
$$

where $\mathbf{g}$ is the required four-component vector function.

Obviously, by theorem 3.3 the vector function $\mathbf{U}$ is a solution of (2.11) for $\mathbf{x} \in$ $\Omega^{+}$. Keeping in mind the boundary condition (3.2) and using (3.21), from (3.33) we obtain, for determining the unknown vector $\mathbf{g}$, a singular integral equation

$$
\mathcal{K}^{(2)} \mathbf{g}(\mathbf{z})=\mathbf{f}(\mathbf{z}) \quad \text { for } \quad \mathbf{z} \in S
$$

By lemma 3.4 the Fredholm's theorems are valid for operator $\mathcal{K}^{(2)}$. We prove that (3.34) is always solvable for an arbitrary vector f. Let us consider the corresponding homogeneous equation

$$
\mathcal{K}^{(2)} \mathbf{g}_{0}(\mathbf{z})=\mathbf{0} \quad \text { for } \quad \mathbf{z} \in S
$$

where $\mathbf{g}_{0}$ is the required four-component vector function. Now we prove that (3.35) has only the trivial solution. 
Indeed, let $\mathbf{g}_{0}$ be a solution of the homogeneous equation (3.35). On the basis of theorem 3.3 and (3.35) the vector $\mathbf{V}(\mathbf{x})=\mathbf{Z}^{(1)}\left(\mathbf{x}, \mathbf{g}_{0}\right)$ is a regular solution of problem $(I I)_{\mathbf{0}, \mathbf{0}}^{+}$. Using theorem 3.1, the problem $(I I)_{\mathbf{0}, \mathbf{0}}^{+}$has only the trivial solution, that is

$$
\mathbf{V}(\mathbf{x})=\mathbf{0} \quad \text { for } \quad \mathbf{x} \in \Omega^{+}
$$

On other hand, by theorem 3.3 and (3.36) we get $\{\mathbf{V}(\mathbf{z})\}^{-}=\mathbf{0}$ for $\mathbf{z} \in S$, i.e., the vector $\mathbf{V}(\mathbf{x})$ is a regular solution of problem $(I)_{\mathbf{0}, \mathbf{0}}^{-}$. On the basis of theorem 3.2 , the problem $(I)_{\mathbf{0}, \mathbf{0}}^{-}$has only the trivial solution, that is

$$
\mathbf{V}(\mathbf{x})=\mathbf{0} \quad \text { for } \quad \mathbf{x} \in \Omega^{-}
$$

By virtue of (3.36), (3.37) and identity (3.21) we obtain

$$
\mathbf{g}_{0}(\mathbf{z})=\left\{\mathbf{P}\left(\mathbf{D}_{\mathbf{z}}, \mathbf{n}\right) \mathbf{V}(\mathbf{z})\right\}^{-}-\left\{\mathbf{P}\left(\mathbf{D}_{\mathbf{z}}, \mathbf{n}\right) \mathbf{V}(\mathbf{z})\right\}^{+}=\mathbf{0} \quad \text { for } \quad \mathbf{z} \in S .
$$

Thus, the homogeneous equation (3.35) has only a trivial solution and therefore (3.34) is always solvable for an arbitrary vector $\mathbf{f}$.

We have thereby proved

Theorem 3.9. If $S \in C^{2, p}$, $\mathbf{f} \in C^{0, p^{\prime}}(S), 0<p^{\prime}<p \leq 1$, then a regular solution of problem $(I I)_{\mathbf{0}, \mathbf{f}}^{+}$exists, is unique and is represented by single-layer potential (3.33), where $\mathbf{g}$ is a solution of the singular integral equation (3.34) which is always solvable for an arbitrary vector $\mathbf{f}$.

Problem $(I)_{\mathbf{0}, \mathbf{f}}^{-}$. We seek a regular solution to problem $(I)_{\mathbf{0}, \mathbf{f}}^{-}$in the form

$$
\mathbf{U}(\mathbf{x})=\mathbf{Z}^{(2)}(\mathbf{x}, \mathbf{h}) \quad \text { for } \quad \mathbf{x} \in \Omega^{-},
$$

where $\mathbf{h}$ is the required four-component vector function.

Obviously, by theorem 3.4 the vector function $\mathbf{U}$ is a solution of (2.11) for $\mathbf{x} \in$ $\Omega^{-}$. Keeping in mind the boundary condition (3.3) and using (3.22), from (3.38) we obtain, for determining the unknown vector $\mathbf{h}$, a singular integral equation

$$
\mathcal{K}^{(3)} \mathbf{h}(\mathbf{z})=\mathbf{f}(\mathbf{z}) \quad \text { for } \quad \mathbf{z} \in S
$$


It has been proved above that the corresponding homogeneous equation (3.35) has only the trivial solution. Hence, it follows that (3.39) is always solvable.

We have thereby proved

Theorem 3.10. If $S \in C^{2, p}$, $\mathbf{f} \in C^{1, p^{\prime}}(S), 0<p^{\prime}<p \leq 1$, then a regular solution of problem $(I)_{\mathbf{0}, \mathbf{f}}^{-}$exists, is unique and is represented by double-layer potential (3.38), where $\mathbf{h}$ is a solution of the singular integral equation (3.39) which is always solvable for an arbitrary vector $\mathbf{f}$.

Remark 3.3. In Pompei and Scalia [102], the BVPs of steady vibrations of the linear theory of elasticity for materials with voids are investigated. The potentials of single layer and double layer are used to reduce the BVPs to singular integral equations for which Fredholm's basic theorems are valid. Existence and uniqueness results for external problems are established. 


\section{Chapter 4}

\section{Solutions of equations in the theory of thermoviscoelasticity for materials with voids}

\subsection{Basic Equations}

The complete system of field equations in the linear theory of thermoviscoelasticity for Kelvin-Voigt material with voids consists of the following equations (Ieşan [62]):

1) The equations of motion (2.1) and (2.2);

2) The constitutive equations

$$
\begin{aligned}
& t_{l j}^{\prime}=2 \mu e_{l j}^{\prime}+\lambda e_{r r}^{\prime} \delta_{l j}+b \varphi^{\prime} \delta_{l j}+2 \mu^{*} \dot{e}_{l j}^{\prime}+\lambda^{*} \dot{e}_{r r}^{\prime} \delta_{l j}+b^{*} \dot{\varphi}^{\prime} \delta_{l j}-\beta \theta^{\prime} \delta_{l j}, \\
& H_{j}^{\prime}=\alpha \varphi_{, j}^{\prime}+\alpha^{*} \dot{\varphi}_{, j}^{\prime}+\tau^{*} \theta_{, j}^{\prime}, \\
& H_{0}^{\prime}=-b e_{r r}^{\prime}-\xi \varphi^{\prime}-\nu^{*} \dot{e}_{r r}^{\prime}-\xi^{*} \dot{\varphi}^{\prime}+m \theta^{\prime}, \\
& \rho \eta^{\prime}=\beta e_{r r}^{\prime}+a \theta^{\prime}+m \varphi^{\prime}, \\
& Q_{j}^{\prime}=k \theta_{j}^{\prime}+\zeta^{*} \dot{\varphi}_{j}^{\prime}, \quad l, j=1,2,3 ;
\end{aligned}
$$


3) The geometrical equations (2.4);

4) The equation of energy

$$
\rho T_{0} \dot{\eta}^{\prime}=Q_{j, j}^{\prime}+\rho \mathcal{F}_{5}^{\prime}
$$

Substituting (4.1) and (2.4) into (2.1), (2.2) and (4.2), we obtain the following system of equations of motion in the linear theory of thermoviscoelasticity for Kelvin-Voigt materials with voids expressed in terms of the displacement vector $\mathbf{u}^{\prime}$, the volume fraction field $\varphi^{\prime}$ and the temperature $\theta^{\prime}$ (Ieşan [62])

$$
\begin{aligned}
& \mu \Delta \mathbf{u}^{\prime}+(\lambda+\mu) \nabla \operatorname{div} \mathbf{u}^{\prime}+b \nabla \varphi^{\prime}+\mu^{*} \Delta \dot{\mathbf{u}}^{\prime}+\left(\lambda^{*}+\mu^{*}\right) \nabla \operatorname{div} \dot{\mathbf{u}}^{\prime} \\
& +b^{*} \nabla \dot{\varphi}^{\prime}-\beta \nabla \theta^{\prime}=\rho\left(\ddot{\mathbf{u}}^{\prime}-\mathcal{F}^{\prime}\right) \\
& (\alpha \Delta-\xi) \varphi^{\prime}-b \operatorname{div} \mathbf{u}^{\prime}+\left(\alpha^{*} \Delta-\xi^{*}\right) \dot{\varphi}^{\prime}-\nu^{*} \operatorname{div} \dot{\mathbf{u}}^{\prime}+\left(\tau^{*} \Delta+m\right) \theta^{\prime} \\
& =\rho_{0} \ddot{\varphi}^{\prime}-\rho \mathcal{F}_{4}^{\prime}, \\
& k \Delta \theta^{\prime}-a T_{0} \dot{\theta}^{\prime}+\left(\zeta^{*} \Delta-m T_{0}\right) \dot{\varphi}^{\prime}-\beta T_{0} \operatorname{div} \dot{\mathbf{u}}^{\prime}=-\rho \mathcal{F}_{5}^{\prime},
\end{aligned}
$$

where $\mathcal{F}^{\prime}=\left(\mathcal{F}_{1}^{\prime}, \mathcal{F}_{2}^{\prime}, \mathcal{F}_{3}^{\prime}\right)$.

If $\mathbf{u}^{\prime}, \varphi^{\prime}, \theta^{\prime}$ and $\mathcal{F}_{j}^{\prime}(j=1,2, \cdots, 5)$ are postulated to have a harmonic time variation, that is,

$$
\left\{\mathbf{u}^{\prime}, \varphi^{\prime}, \theta^{\prime}, \mathcal{F}_{j}^{\prime}\right\}(\mathbf{x}, t)=\operatorname{Re}\left[\left\{\mathbf{u}, \varphi, \theta, \mathcal{F}_{j}\right\}(\mathbf{x}) e^{-i \omega t}\right]
$$

then from system of equations of motion (4.3) we obtain the following system of equations of steady vibrations in the linear theory of thermoviscoelasticity for 
Kelvin-Voigt materials with voids

$$
\begin{aligned}
& \left(\mu_{1} \Delta+\rho \omega^{2}\right) \mathbf{u}+\left(\lambda_{1}+\mu_{1}\right) \nabla \operatorname{div} \mathbf{u}+b_{1} \nabla \varphi-\beta \nabla \theta=-\rho \mathcal{F}, \\
& \left(\alpha_{1} \Delta+\xi_{2}\right) \varphi+\left(\tau^{*} \Delta+m\right) \theta-\nu_{1} \operatorname{div} \mathbf{u}=-\rho \mathcal{F}_{4}, \\
& \left(k \Delta+c_{1}\right) \theta+\left(\zeta_{1} \Delta+m_{1}\right) \varphi+\beta_{1} \operatorname{div} \mathbf{u}=-\rho \mathcal{F}_{5},
\end{aligned}
$$

where $\lambda_{1}, \mu_{1}, b_{1}, \alpha_{1}, \nu_{1}, \xi_{1}, \xi_{2}$ are given by (2.7) and

$$
c_{1}=i \omega a T_{0}, \quad \zeta_{1}=-i \omega \zeta^{*}, \quad m_{1}=-i \omega m T_{0}, \quad \beta_{1}=-i \omega \beta T_{0} .
$$

Obviously, (4.4) is the system of partial differential equations with complex coefficients in which there are 21 real parameters: $\lambda, \lambda^{*}, \mu, \mu^{*}, b, b^{*}, \alpha, \alpha^{*}, \xi, \xi^{*}, \nu^{*}, k$, $T_{0}, \tau^{*}, m, a, \beta, \zeta^{*}, \omega, \rho$ and $\rho_{1}$.

In the absence of the body force, the extrinsic equilibrated body force and the heat supply from (4.4) we obtain the following system of homogeneous equations of steady vibrations in the linear theory of thermoviscoelasticity for Kelvin-Voigt materials with voids

$$
\begin{aligned}
& \left(\mu_{1} \Delta+\rho \omega^{2}\right) \mathbf{u}+\left(\lambda_{1}+\mu_{1}\right) \nabla \operatorname{div} \mathbf{u}+b_{1} \nabla \varphi-\beta \nabla \theta=\mathbf{0}, \\
& \left(\alpha_{1} \Delta+\xi_{2}\right) \varphi+\left(\tau^{*} \Delta+m\right) \theta-\nu_{1} \operatorname{div} \mathbf{u}=0, \\
& \left(k \Delta+c_{1}\right) \theta+\left(\zeta_{1} \Delta+m_{1}\right) \varphi+\beta_{1} \operatorname{div} \mathbf{u}=0,
\end{aligned}
$$


We introduce the matrix differential operator

$$
\begin{aligned}
& \mathcal{A}\left(\mathbf{D}_{\mathbf{x}}\right)=\left(\mathcal{A}_{p q}\left(\mathbf{D}_{\mathbf{x}}\right)\right)_{5 \times 5}, \\
& \mathcal{A}_{l j}\left(\mathbf{D}_{\mathbf{x}}\right)=\left(\mu_{1} \Delta+\rho \omega^{2}\right) \delta_{l j}+\left(\lambda_{1}+\mu_{1}\right) \frac{\partial^{2}}{\partial x_{l} \partial x_{j}}, \\
& \mathcal{A}_{l 4}\left(\mathbf{D}_{\mathbf{x}}\right)=b_{1} \frac{\partial}{\partial x_{l}}, \quad A_{l 5}\left(\mathbf{D}_{\mathbf{x}}\right)=-\beta \frac{\partial}{\partial x_{l}}, \\
& \mathcal{A}_{4 l}\left(\mathbf{D}_{\mathbf{x}}\right)=-\nu_{1} \frac{\partial}{\partial x_{l}}, \quad \mathcal{A}_{44}\left(\mathbf{D}_{\mathbf{x}}\right)=\alpha_{1} \Delta+\xi_{2}, \\
& \mathcal{A}_{45}\left(\mathbf{D}_{\mathbf{x}}\right)=\tau^{*} \Delta+m, \quad \mathcal{A}_{5 l}\left(\mathbf{D}_{\mathbf{x}}\right)=\beta_{1} \frac{\partial}{\partial x_{l}}, \\
& \mathcal{A}_{54}\left(\mathbf{D}_{\mathbf{x}}\right)=\zeta_{1} \Delta+m_{1}, \quad \mathcal{A}_{55}\left(\mathbf{D}_{\mathbf{x}}\right)=k \Delta+c_{1}, \quad l, j=1,2,3 .
\end{aligned}
$$

The systems (4.4) and (4.6) can be written as

$$
\mathcal{A}\left(\mathbf{D}_{\mathbf{x}}\right) \mathbf{V}(\mathbf{x})=\mathbf{F}(\mathbf{x})
$$

and

$$
\mathcal{A}\left(\mathbf{D}_{\mathbf{x}}\right) \mathbf{V}(\mathbf{x})=\mathbf{0}
$$

where $\mathbf{V}=(\mathbf{u}, \varphi, \theta)$ and $\mathbf{F}=\left(-\rho \mathcal{F},-\rho \mathcal{F}_{4},-\rho \mathcal{F}_{5}\right)$ are five-component vector functions.

In the follows we assume that the constitutive coefficients satisfy the conditions (Ieşan [62])

$$
\begin{aligned}
& \mu^{*}>0, \quad 3 \lambda^{*}+2 \mu^{*}>0, \quad \alpha^{*}>0, \quad a>0, \quad k>0, \\
& \left(3 \lambda^{*}+2 \mu^{*}\right) \xi^{*}>\frac{3}{4}\left(b^{*}+\nu^{*}\right)^{2},
\end{aligned}
$$




\subsection{Fundamental solution}

In this section the fundamental solution of system (4.6) is constructed and its basic properties are established.

Definition 4.1. The fundamental solution of the system (4.6) (the fundamental matrix of operator $\left.\mathcal{A}\left(\mathbf{D}_{\mathbf{x}}\right)\right)$ is the matrix $\boldsymbol{\Theta}(\mathbf{x})=\left(\Theta_{l j}(\mathbf{x})\right)_{5 \times 5}$ satisfying condition in the class of generalized functions (see Hörmander [87])

$$
\mathcal{A}\left(\mathbf{D}_{\mathbf{x}}\right) \boldsymbol{\Theta}(\mathbf{x})=\delta(\mathbf{x}) \mathbf{J}^{\prime}
$$

where $\mathbf{x} \in \mathbb{R}^{3}$.

In the sequel we use the following differential operators

$$
\mathcal{B}(\Delta)=\left(\mathcal{B}_{l j}(\Delta)\right)_{3 \times 3}, \quad \Lambda_{1}(\Delta)=\frac{1}{\mu_{2} k_{0}} \operatorname{det} \mathcal{B}(\Delta),
$$

where

$$
\begin{array}{lcc}
\mathcal{B}_{11}(\Delta)=\mu_{2} \Delta+\rho \omega^{2}, & \mathcal{B}_{12}(\Delta)=-\nu_{1} \Delta, & \mathcal{B}_{13}(\Delta)=\beta_{1} \Delta, \\
\mathcal{B}_{21}(\Delta)=b_{1}, & \mathcal{B}_{22}(\Delta)=\alpha_{1} \Delta+\xi_{2}, & \mathcal{B}_{23}(\Delta)=\zeta_{1} \Delta+m_{1}, \\
\mathcal{B}_{31}(\Delta)=-\beta, & \mathcal{B}_{32}(\Delta)=\tau^{*} \Delta+m, & \mathcal{B}_{33}(\Delta)=k \Delta+c_{1}, \\
\mu_{2}=\lambda_{1}+2 \mu_{1}, & k_{0}=\alpha_{1} k-\zeta_{1} \tau^{*} .
\end{array}
$$

Taking into account (2.7) and (4.10) we have $\mu_{1} \mu_{2} k_{0} \neq 0$.

It is easily seen that

$$
\Lambda_{1}(\Delta)=\left(\Delta+\kappa_{1}^{2}\right)\left(\Delta+\kappa_{2}^{2}\right)\left(\Delta+\kappa_{3}^{2}\right)
$$

where $\kappa_{1}^{2}, \kappa_{2}^{2}$ and $\kappa_{3}^{2}$ are the roots of equation $\Lambda_{1}(-\kappa)=0$ (with respect to $\kappa$ ). In view of (2.7) and (4.5) we get

$$
\xi_{2} c_{1}-m m_{1}=-a T_{0} \xi^{*} \omega^{2}+i \omega T_{0}\left[a\left(\rho_{0} \omega^{2}-\xi\right)+m^{2}\right]
$$


and by virtue of (4.10) we have

$$
\xi_{2} c_{1}-m m_{1} \neq 0
$$

Obviously, from (4.13) it follows that $\kappa_{j} \neq 0(j=1,2,3)$.

In addition, it is also assumed that $\operatorname{Im} \kappa_{j}>0$ and $\kappa_{j}^{2} \neq \kappa_{l}^{2}$, where $j, l=$ $1,2,3,4, j \neq l$ and $\kappa_{4}^{2}=\tau_{3}^{2}=\frac{\rho \omega^{2}}{\mu_{1}}$.

We consider the system of nonhomogeneous equations

$$
\begin{aligned}
& \left(\mu_{1} \Delta+\rho \omega^{2}\right) \mathbf{u}+\left(\lambda_{1}+\mu_{1}\right) \nabla \operatorname{div} \mathbf{u}-\nu_{1} \nabla \varphi+\beta_{1} \nabla \theta=\mathcal{F}, \\
& b_{1} \operatorname{div} \mathbf{u}+\left(\alpha_{1} \Delta+\xi_{2}\right) \varphi+\left(\zeta_{1} \Delta+m_{1}\right) \theta=\mathcal{F}_{4}, \\
& -\beta \operatorname{div} \mathbf{u}+\left(\tau^{*} \Delta+m\right) \varphi+\left(k \Delta+c_{1}\right) \theta=\mathcal{F}_{5},
\end{aligned}
$$

where $\mathcal{F}=\left(\mathcal{F}_{1}, \mathcal{F}_{2}, \mathcal{F}_{3}\right)$. As one may easily verify, system (4.14) may be written in the form

$$
\mathcal{A}^{\top}\left(\mathbf{D}_{\mathbf{x}}\right) \mathbf{V}(\mathbf{x})=\mathbf{F}(\mathbf{x})
$$

where $\mathcal{A}^{\top}$ is the transpose of matrix $\mathcal{A}, \mathbf{F}=\left(\mathcal{F}, \mathcal{F}_{4}, \mathcal{F}_{5}\right)$ is five-component vector function on $\mathbb{R}^{3}$, and $\mathrm{x} \in \mathbb{R}^{3}$.

Applying the operator div to the first equation of (4.14) from system (4.14) we obtain

$$
\begin{aligned}
& \left(\mu_{2} \Delta+\rho \omega^{2}\right) \operatorname{div} \mathbf{u}-\nu_{1} \Delta \varphi+\beta_{1} \Delta \theta=\operatorname{div} \mathcal{F} \\
& b_{1} \operatorname{div} \mathbf{u}+\left(\alpha_{1} \Delta+\xi_{2}\right) \varphi+\left(\zeta_{1} \Delta+m_{1}\right) \theta=\mathcal{F}_{4}, \\
& -\beta \operatorname{div} \mathbf{u}+\left(\tau^{*} \Delta+m\right) \varphi+\left(k \Delta+c_{1}\right) \theta=\mathcal{F}_{5} .
\end{aligned}
$$

Its matrix form is

$$
\mathcal{B}(\Delta) \mathbf{V}^{\prime}(\mathbf{x})=\mathbf{F}^{\prime}(\mathbf{x})
$$

where $\mathbf{V}^{\prime}=(\operatorname{div} \mathbf{u}, \varphi, \theta)$ and $\mathbf{F}^{\prime}=\left(F_{1}, F_{2}, F_{3}\right)=\left(\operatorname{div} \mathcal{F}, \mathcal{F}_{4}, \mathcal{F}_{5}\right)$. The system (4.16) implies

$$
\Lambda_{1}(\Delta) \mathbf{V}^{\prime}=\Phi
$$


where

$$
\mathbf{\Phi}=\left(\Phi_{1}, \Phi_{2}, \Phi_{3}\right), \quad \Phi_{j}=\frac{1}{\mu_{2} k_{0}} \mathcal{B}_{l j}^{*} F_{l}, \quad j=1,2,3
$$

and $\mathcal{B}_{l j}^{*}$ is the cofactor of the element $\mathcal{B}_{l j}$ of the matrix $\mathcal{B}$.

Now applying the operators $\Lambda_{1}(\Delta)$ to the first equation of (4.14) and taking into account (4.17) we obtain

$$
\Lambda_{1}(\Delta)\left(\mu_{1} \Delta+\rho \omega^{2}\right) \mathbf{u}+\left(\lambda_{1}+\mu_{1}\right) \nabla \Phi_{1}-\nu_{1} \nabla \Phi_{2}+\beta_{1} \nabla \Phi_{3}=\Lambda_{1}(\Delta) \mathcal{F}
$$

and whence follows

$$
\Lambda_{2}(\Delta) \mathbf{u}=\Phi^{\prime}
$$

where

$$
\Lambda_{2}(\Delta)=\Lambda_{1}(\Delta)\left(\Delta+\kappa_{4}^{2}\right)
$$

and

$$
\boldsymbol{\Phi}^{\prime}=\frac{1}{\mu_{1}}\left[\Lambda_{1}(\Delta) \mathcal{F}-\left(\lambda_{1}+\mu_{1}\right) \nabla \Phi_{1}+\nu_{1} \nabla \Phi_{2}-\beta_{1} \nabla \Phi_{3}\right] .
$$

By virtue of (4.17) and (4.19) we get

$$
\Lambda^{\prime}(\Delta) \mathbf{V}(\mathbf{x})=\tilde{\boldsymbol{\Phi}}(\mathbf{x})
$$

where $\tilde{\boldsymbol{\Phi}}=\left(\boldsymbol{\Phi}^{\prime}, \Phi_{2}, \Phi_{3}\right)$ and

$$
\begin{aligned}
& \Lambda^{\prime}(\Delta)=\left(\Lambda_{l j}^{\prime}(\Delta)\right)_{5 \times 5}, \quad \Lambda_{11}^{\prime}(\Delta)=\Lambda_{22}^{\prime}(\Delta)=\Lambda_{33}^{\prime}(\Delta)=\Lambda_{2}(\Delta), \\
& \Lambda_{44}^{\prime}(\Delta)=\Lambda_{55}^{\prime}(\Delta)=\Lambda_{1}(\Delta), \quad \Lambda_{l j}^{\prime}(\Delta)=0, \\
& l, j=1,2, \cdots, 5, \quad l \neq j .
\end{aligned}
$$


In view of (4.18) and (4.20) we have

$$
\begin{aligned}
& \Phi^{\prime}=\left[\frac{1}{\mu_{1}} \Lambda_{1}(\Delta) \mathbf{I}+n_{11}(\Delta) \nabla \operatorname{div}\right] \mathcal{F}+n_{21}(\Delta) \nabla \mathcal{F}_{4}+n_{31}(\Delta) \nabla \mathcal{F}_{5}, \\
& \Phi_{2}=n_{12}(\Delta) \operatorname{div} \mathcal{F}+n_{22}(\Delta) \mathcal{F}_{4}+n_{32}(\Delta) \mathcal{F}_{5}, \\
& \Phi_{3}=n_{13}(\Delta) \operatorname{div} \mathcal{F}+n_{32}(\Delta) \mathcal{F}_{4}+n_{33}(\Delta) \mathcal{F}_{5},
\end{aligned}
$$

where

$$
\begin{aligned}
& n_{l 1}(\Delta)=\frac{1}{\mu_{1} \mu_{2} k_{0}}\left[-\left(\lambda_{1}+\mu_{1}\right) \mathcal{B}_{l 1}^{*}(\Delta)+\nu_{1} \mathcal{B}_{l 2}^{*}(\Delta)-\beta_{1} \mathcal{B}_{l 3}^{*}(\Delta)\right], \\
& n_{l j}(\Delta)=\frac{1}{\mu_{2} k_{0}} \mathcal{B}_{l j}^{*}(\Delta), \quad l=1,2,3, \quad j=2,3 .
\end{aligned}
$$

Obviously, from (4.22) we obtain

$$
\tilde{\boldsymbol{\Phi}}(\mathbf{x})=\mathbf{M}^{\top}\left(\mathbf{D}_{\mathbf{x}}\right) \mathbf{F}(\mathbf{x})
$$

where

$$
\begin{array}{ll}
\mathbf{M}\left(\mathbf{D}_{\mathbf{x}}\right)=\left(M_{l j}\left(\mathbf{D}_{\mathbf{x}}\right)\right)_{5 \times 5}, & M_{l j}\left(\mathbf{D}_{\mathbf{x}}\right)=\frac{1}{\mu_{1}} \Lambda_{1}(\Delta) \delta_{l j}+n_{11}(\Delta) \frac{\partial^{2}}{\partial x_{l} \partial x_{j}} \\
M_{l p}\left(\mathbf{D}_{\mathbf{x}}\right)=n_{1 ; p-2}(\Delta) \frac{\partial}{\partial x_{l}}, & M_{p l}\left(\mathbf{D}_{\mathbf{x}}\right)=n_{p-2 ; 1}(\Delta) \frac{\partial}{\partial x_{l}} \\
M_{p q}\left(\mathbf{D}_{\mathbf{x}}\right)=n_{p-2 ; q-2}(\Delta), & l, j=1,2,3, \quad p, q=4,5
\end{array}
$$

By virtue of (4.15) and (4.23) from (4.21) it follows that $\Lambda^{\prime} \mathbf{V}=\mathbf{M}^{\top} \mathcal{A}^{\top} \mathbf{V}$. It is obvious that $\mathbf{M}^{\top} \mathcal{A}^{\top}=\boldsymbol{\Lambda}^{\prime}$, and therefore,

$$
\mathcal{A}\left(\mathbf{D}_{\mathbf{x}}\right) \mathbf{M}\left(\mathbf{D}_{\mathbf{x}}\right)=\Lambda^{\prime}(\Delta)
$$


We introduce the notations

$$
\begin{aligned}
& \mathbf{Y}^{\prime}(\mathbf{x})=\left(Y_{l j}^{\prime}(\mathbf{x})\right)_{5 \times 5}, \quad Y_{11}^{\prime}(\mathbf{x})=Y_{22}^{\prime}(\mathbf{x})=Y_{33}^{\prime}(\mathbf{x})=\sum_{j=1}^{4} c_{1 j}^{\prime} \gamma_{j}^{\prime}(\mathbf{x}), \\
& Y_{44}^{\prime}(\mathbf{x})=Y_{55}^{\prime}(\mathbf{x})=c_{2 j}^{\prime} \gamma_{j}^{\prime}(\mathbf{x}), \quad Y_{l j}^{\prime}(\mathbf{x})=0, \\
& l, j=1,2, \cdots, 5, \quad l \neq j,
\end{aligned}
$$

where

$$
\begin{aligned}
& \gamma_{j}^{\prime}(\mathbf{x})=-\frac{e^{i \kappa_{j}|\mathbf{x}|}}{4 \pi|\mathbf{x}|}, \quad c_{1 j}^{\prime}=\prod_{l=1 ; l \neq j}^{4}\left(\kappa_{l}^{2}-\kappa_{j}^{2}\right)^{-1}, \\
& c_{2 j}^{\prime}=\prod_{l=1 ; l \neq j}^{3}\left(\kappa_{l}^{2}-\kappa_{j}^{2}\right)^{-1}, \quad j=1,2,3,4 .
\end{aligned}
$$

Obviously, $\mathbf{Y}^{\prime}$ is the fundamental matrix of operator $\boldsymbol{\Lambda}^{\prime}$, that is,

$$
\mathbf{\Lambda}^{\prime}(\Delta) \mathbf{Y}^{\prime}(\mathbf{x})=\delta(\mathbf{x}) \mathbf{J}^{\prime}
$$

We define the matrix $\Theta=\left(\Theta_{p q}\right)_{5 \times 5}$ by

$$
\Theta(\mathbf{x})=\mathbf{M}\left(\mathbf{D}_{\mathbf{x}}\right) \mathbf{Y}^{\prime}(\mathbf{x})
$$

In view of (4.25), (4.27) and (4.28) we get

$$
\mathcal{A}\left(\mathbf{D}_{\mathbf{x}}\right) \boldsymbol{\Theta}(\mathbf{x})=\mathcal{A}\left(\mathbf{D}_{\mathbf{x}}\right) \mathbf{M}\left(\mathbf{D}_{\mathbf{x}}\right) \mathbf{Y}^{\prime}(\mathbf{x})=\mathbf{\Lambda}^{\prime}(\Delta) \mathbf{Y}^{\prime}(\mathbf{x})=\delta(\mathbf{x}) \mathbf{J}^{\prime}
$$

Hence, $\boldsymbol{\Theta}(\mathbf{x})$ is a solution of (4.11). We have thereby proved the following theorem.

Theorem 4.1. If condition (4.10) is satisfied, then the matrix $\mathbf{\Theta}(\mathbf{x})$ defined by (4.28) is the fundamental solution of system (4.6), where $\mathbf{M}\left(\mathbf{D}_{\mathbf{x}}\right)$ and $\mathbf{Y}^{\prime}(\mathbf{x})$ are given by (4.24) and (4.26), respectively.

Remark 4.1. The cases when some of $\kappa_{j}$ are equal must be considered separately 
by passing to the limit in the expressions $Y_{11}^{\prime}$ and $Y_{44}^{\prime}$ (see (4.26)).

Theorem 4.1 leads to the following results.

Corollary 4.1. If condition (4.10) is satisfied, then each column of the matrix $\boldsymbol{\Theta}(\mathbf{x})$ is the solution of the homogeneous equation (4.6) at every point $\mathbf{x} \in R^{3}$ except the origin.

Corollary 4.2. If condition (4.10) is satisfied, then the fundamental solution of the system

$$
\begin{aligned}
& \mu_{1} \Delta \mathbf{u}+\left(\lambda_{1}+\mu_{1}\right) \nabla \operatorname{div} \mathbf{u}=\mathbf{0}, \\
& \alpha_{1} \Delta \varphi+\tau^{*} \Delta \theta=0, \quad k \Delta \theta+\zeta_{1} \Delta \varphi=0,
\end{aligned}
$$

is the matrix $\Psi^{\prime}=\left(\Psi_{l j}^{\prime}\right)_{5 \times 5}$, where

$$
\begin{aligned}
& \Psi_{l j}^{\prime}(\mathbf{x})=\left(\frac{1}{\mu_{1}} \Delta \delta_{l j}-\frac{\lambda_{1}+\mu_{1}}{\mu_{1} \mu_{2}} \frac{\partial^{2}}{\partial x_{l} \partial x_{j}}\right) \gamma_{4}(\mathbf{x}), \\
& \Psi_{l p}^{\prime}(\mathbf{x})=\Psi_{p l}^{\prime}(\mathbf{x})=0, \quad \Psi_{44}^{\prime}(\mathbf{x})=\frac{1}{k_{0}} k \gamma_{5}(\mathbf{x}), \\
& \Psi_{45}^{\prime}(\mathbf{x})=-\frac{1}{k_{0}} \tau^{*} \gamma_{5}(\mathbf{x}), \quad \Psi_{54}^{\prime}(\mathbf{x})=-\frac{1}{k_{0}} \zeta_{1} \gamma_{5}(\mathbf{x}), \\
& \Psi_{55}^{\prime}(\mathbf{x})=\frac{1}{k_{0}} \alpha_{1} \gamma_{5}(\mathbf{x}), \quad l, j=1,2,3, \quad p=4,5
\end{aligned}
$$

$\gamma_{4}(\mathbf{x})$ and $\gamma_{5}(\mathbf{x})$ are given in corollary 2.2.

Clearly (see Kupradze et al. [83]), the relations

$$
\begin{aligned}
& \Psi_{l j}^{\prime}(\mathbf{x})=O\left(|\mathbf{x}|^{-1}\right), \quad \Psi_{p q}^{\prime}(\mathbf{x})=O\left(|\mathbf{x}|^{-1}\right) \\
& \frac{\partial}{\partial x_{m}} \Psi_{l j}^{\prime}(\mathbf{x})=O\left(|\mathbf{x}|^{-2}\right), \quad \frac{\partial}{\partial x_{m}} \Psi_{p q}^{\prime}(\mathbf{x})=O\left(|\mathbf{x}|^{-2}\right) \\
& \Psi_{l p}^{\prime}(\mathbf{x})=\Psi_{p l}^{\prime}(\mathbf{x})=0, \quad l, j, m=1,2,3, \quad p, q=4,5
\end{aligned}
$$

hold in a neighborhood of the origin. 
On the basis of theorem 4.1 and corollary 4.2 we obtain the following result.

Theorem 4.2. If condition (4.10) is satisfied, then the relations

$$
\begin{aligned}
& \Theta_{l j}(\mathbf{x})=O\left(|\mathbf{x}|^{-1}\right), \quad \Theta_{p q}(\mathbf{x})=O\left(|\mathbf{x}|^{-1}\right), \\
& \Theta_{l p}(\mathbf{x})=\text { const }+O\left(|\mathbf{x}|^{-1}\right), \quad \Theta_{p l}(\mathbf{x})=\text { const }+O\left(|\mathbf{x}|^{-1}\right), \\
& \Theta_{l j}(\mathbf{x})-\Psi_{l j}^{\prime}(\mathbf{x})=\text { const }+O(|\mathbf{x}|), \\
& \Theta_{p q}(\mathbf{x})-\Psi_{p q}^{\prime}(\mathbf{x})=\mathrm{const}+O(|\mathbf{x}|), \\
& \frac{\partial^{r}}{\partial x_{1}^{r_{1}} \partial x_{2}^{r_{2}} \partial x_{3}^{r_{3}}}\left[\Theta_{l j}(\mathbf{x})-\Psi_{l j}^{\prime}(\mathbf{x})\right]=O\left(|\mathbf{x}|^{1-m}\right), \\
& \frac{\partial^{r}}{\partial x_{1}^{r_{1}} \partial x_{2}^{r_{2}} \partial x_{3}^{r_{3}}}\left[\Theta_{p q}(\mathbf{x})-\Psi_{p q}^{\prime}(\mathbf{x})\right]=O\left(|\mathbf{x}|^{1-m}\right)
\end{aligned}
$$

hold in a neighborhood of the origin, where $r=r_{1}+r_{2}+r_{3}, r \geq 1, r_{l} \geq 0, l, j=$ $1,2,3$ and $p, q=4,5$.

Thus, $\Psi^{\prime}(\mathbf{x})$ is the singular part of the fundamental matrix $\Theta(\mathbf{x})$ in the neighborhood of the origin.

\subsection{Galerkin type solution}

In this section the Galerkin type solution of the system (4.4) is obtained, and the formula of representation of general solution of the system of homogeneous equations (4.6) in terms of six metaharmonic functions is established.

Let $Q_{1}, Q_{2}, \cdots, Q_{5}$ be functions on $\Omega, \mathbf{Q}^{(1)}=\left(Q_{1}, Q_{2}, Q_{3}\right)^{\top}$. Obviously, ma- 
trix $\mathbf{M}$ defined by (4.24) can be rewritten in the form

$$
\begin{aligned}
& \mathbf{M}=\left(M_{l j}\right)_{5 \times 5}=\left(\begin{array}{lll}
\mathbf{M}^{(1)} & \mathbf{M}^{(2)} & \mathbf{M}^{(3)} \\
\mathbf{M}^{(4)} & M_{44} & M_{45} \\
\mathbf{M}^{(5)} & M_{54} & M_{55}
\end{array}\right)_{5 \times 5}, \\
& \mathbf{M}^{(1)}=\left(M_{l j}\right)_{3 \times 3}, \quad \mathbf{M}^{(2)}=\left(M_{l 4}\right)_{3 \times 1}, \quad \mathbf{M}^{(3)}=\left(M_{l 5}\right)_{3 \times 1}, \\
& \mathbf{M}^{(4)}=\left(M_{4 l}\right)_{1 \times 3}, \quad \mathbf{M}^{(5)}=\left(M_{5 l}\right)_{1 \times 3},
\end{aligned}
$$

The next theorem provides a Galerkin type solution to system (4.4).

Theorem 4.3. Let

$$
\begin{aligned}
& \mathbf{u}=\mathbf{M}^{(1)} \mathbf{Q}^{(1)}+\mathbf{M}^{(2)} Q_{4}+\mathbf{M}^{(3)} Q_{5}, \\
& \varphi=\mathbf{M}^{(4)} \mathbf{Q}^{(1)}+M_{44} Q_{4}+M_{45} Q_{5}, \\
& \theta=\mathbf{M}^{(5)} \mathbf{Q}^{(1)}+M_{54} Q_{4}+M_{55} Q_{5},
\end{aligned}
$$

where $\mathbf{Q}^{(1)}, Q_{4}$ and $Q_{5}$ satisfy the following equations

$$
\Lambda_{2}(\Delta) \mathbf{Q}^{(1)}=-\rho \mathcal{F}, \quad \Lambda_{1} Q_{p}=-\rho \mathcal{F}_{p}, \quad p=4,5
$$

on $\Omega$, then the vector $\mathbf{V}=(\mathbf{u}, \varphi, \theta)$ is the solution of (4.4).

Proof. On the basis of (4.29) from (4.30) we have

$$
\mathbf{V}(\mathbf{x})=\mathbf{M}\left(\mathbf{D}_{\mathbf{x}}\right) \mathbf{Q}(\mathbf{x})
$$

On the other hand, from (4.31) we get

$$
\Lambda^{\prime}(\Delta) \mathbf{Q}(\mathbf{x})=\mathbf{F}(\mathbf{x})
$$

where $\mathbf{F}=\left(-\rho \mathcal{F},-\rho \mathcal{F}_{4},-\rho \mathcal{F}_{5}\right)$ is a five-component vector function. 
By virtue of (4.25), (4.32) and (4.33) we obtain

$$
\mathcal{A}\left(\mathbf{D}_{\mathbf{x}}\right) \mathbf{V}=\mathcal{A}\left(\mathbf{D}_{\mathbf{x}}\right) \mathbf{M}\left(\mathbf{D}_{\mathbf{x}}\right) \mathbf{Q}=\Lambda^{\prime}(\Delta) \mathbf{Q}=\mathbf{F}
$$

$\diamond$ i.e,

Let $\tilde{\boldsymbol{\Theta}}(\mathbf{x})=\left(\tilde{\Theta}_{l j}(\mathbf{x})\right)_{5 \times 5}$ be the fundamental matrix of the operator $\mathbf{M}\left(\mathbf{D}_{\mathbf{x}}\right)$,

$$
\mathbf{M}\left(\mathbf{D}_{\mathbf{x}}\right) \tilde{\boldsymbol{\Theta}}(\mathbf{x})=\delta(\mathbf{x}) \mathbf{J}^{\prime}
$$

We can construct the matrix $\tilde{\boldsymbol{\Theta}}(\mathbf{x})$ by elementary functions quite similarly to matrix $\boldsymbol{\Theta}(\mathbf{x})$ in the previous section.

Theorem 4.4. Let $\Omega$ be a finite domain in $R^{3}$. If $\mathbf{V}=(\mathbf{u}, \varphi, \theta)$ is a solution of (4.8), then $\mathbf{V}$ is represented by (4.30), where $\mathbf{Q}^{(1)}, Q_{4}$ and $Q_{5}$ satisfy the equations (4.31).

Proof. Let $\mathbf{V}=(\mathbf{u}, \varphi, \theta)$ be a solution of (4.8). On the basis of (4.34) the volume potential

$$
\mathbf{Q}(\mathbf{x})=\int_{\Omega} \tilde{\mathbf{\Theta}}(\mathbf{x}-\mathbf{y}) \mathbf{V}(\mathbf{y}) d \mathbf{y}
$$

is a solution of the following equation

$$
\mathbf{M}\left(\mathbf{D}_{\mathbf{x}}\right) \mathbf{Q}(\mathbf{x})=\mathbf{V}(\mathbf{x})
$$

Hence, $\mathbf{V}$ is represented by (4.30).

On the other hand by virtue of (4.8), (4.25) and (4.35) we get

$$
\Lambda^{\prime}(\Delta) \mathbf{Q}(\mathbf{x})=\mathcal{A}\left(\mathbf{D}_{\mathbf{x}}\right) \mathbf{M}\left(\mathbf{D}_{\mathbf{x}}\right) \mathbf{Q}(\mathbf{x})=\mathcal{A}\left(\mathbf{D}_{\mathbf{x}}\right) \mathbf{V}(\mathbf{x})=\mathbf{F}(\mathbf{x})
$$

Thus, $\mathbf{Q}^{(1)}, Q_{4}$ and $Q_{5}$ satisfy the equations (4.31) $\diamond$

By theorems 4.3 and 4.4 the completeness of Galerkin type solution of system (4.4) is proved. 


\subsection{Representation of general solution of system of homogeneous equations}

In this section the general solution of the system of homogeneous equations (4.6) is represented by six metaharmonic functions.

Let $\mathbf{V}=(\mathbf{u}, \varphi, \theta)$ be a solution of (4.6). Applying the operator div to the first equation of (4.6) from system (4.6) we get

$$
\begin{aligned}
& \left(\mu_{2} \Delta+\rho \omega^{2}\right) v+b_{1} \Delta \varphi-\beta \Delta \theta=0, \\
& -\nu_{1} v+\left(\alpha_{1} \Delta+\xi_{2}\right) \varphi+\left(\tau^{*} \Delta+m\right) \theta=0, \\
& \beta_{1} v+\left(\zeta_{1} \Delta+m_{1}\right) \varphi+\left(k \Delta+c_{1}\right) \theta=0,
\end{aligned}
$$

where $v=\operatorname{div} \mathbf{u}$.

In the sequel we need the following lemmas.

Lemma 4.1 If $(v, \varphi, \theta)$ is a solution of (4.36), then

$$
\begin{aligned}
& {\left[k_{0}\left(\mu_{2} \Delta+\rho \omega^{2}\right)+a_{11}\right] v+a_{12} \varphi+a_{13} \theta=0,} \\
& a_{21} v+\left(k_{0} \Delta+a_{22}\right) \varphi+a_{23} \theta=0, \\
& a_{31} v+a_{32} \theta+\left(k_{0} \Delta+a_{33}\right) \varphi=0,
\end{aligned}
$$

where

$$
\begin{array}{lll}
a_{11}=a_{31} \beta-a_{21} b_{1}, & a_{12}=a_{32} \beta-a_{22} b_{1}, & a_{13}=a_{33} \beta-a_{23} b_{1}, \\
a_{21}=-\left(\nu_{1} k+\beta_{1} \tau^{*}\right), & a_{21}=\xi_{2} k-m_{1} \tau^{*}, & a_{23}=m k-c_{1} \tau^{*}, \\
a_{31}=\alpha_{1} \beta_{1}+\nu_{1} \zeta_{1}, & a_{32}=\alpha_{1} m_{1}-\zeta_{1} \xi_{2}, & a_{33}=\alpha_{1} c_{1}-\zeta_{1} m .
\end{array}
$$

Proof. Multiplying the second and third equations of (4.36) by $k$ and $\tau^{*}$, respectively, and subtracting we get the second equation of (4.37). Multiplying the 
third and second equations of (4.36) by $\alpha_{1}$ and $\zeta_{1}$, respectively, and subtracting we obtain the third equation of (4.37). Now multiplying the first equation of (4.36) by $k_{0}$ and substituting here the second and third equations of (4.37) we finally have the first equation of (4.37). $\diamond$

We introduce the notations

$$
\begin{aligned}
& N_{1}(\Delta)=-a_{13}\left[\left(k_{0} \Delta+a_{33}\right)\left(k_{0} \mu_{2} \Delta+k_{0} \rho \omega^{2}+a_{11}\right)-a_{13} a_{31}\right] \\
& -k_{0} a_{12} a_{23}\left(\mu_{2} \Delta+\rho \omega^{2}\right)+a_{12}\left(a_{21} a_{13}-a_{11} a_{23}\right), \\
& N_{2}(\Delta)=a_{12}\left[\left(k_{0} \Delta+a_{22}\right)\left(k_{0} \mu_{2} \Delta+k_{0} \rho \omega^{2}+a_{11}\right)-a_{12} a_{21}\right] \\
& +k_{0} a_{13} a_{32}\left(\mu_{2} \Delta+\rho \omega^{2}\right)+a_{13}\left(a_{11} a_{32}-a_{12} a_{31}\right), \\
& d_{0}=a_{12}\left(a_{12} a_{23}-a_{13} a_{22}\right)+a_{13}\left(a_{12} a_{33}-a_{13} a_{32}\right) \\
& =k_{0}\left(m m_{1}-\xi_{2} c_{1}\right)\left(a_{12} \beta+a_{13} b_{1}\right) \text {. }
\end{aligned}
$$

By direct calculation from (4.38) and (4.39) we obtain the following identity

$$
a_{12} N_{1}(\Delta)+a_{13} N_{2}(\Delta)+d_{0}\left[k_{0}\left(\mu_{2} \Delta+\rho \omega^{2}\right)+a_{11}\right]=0 .
$$

Lemma 4.2 If $(v, \varphi, \theta)$ is a solution of (4.36), then

$$
d_{0} \varphi=N_{1}(\Delta) v, \quad d_{0} \theta=N_{2}(\Delta) v
$$

Proof. Applying the operator $k_{0} \Delta+a_{33}$ to the first equation of (4.37) and substituting here the third equation of (4.37) we obtain

$$
\left(k_{0} \Delta+a_{33}\right)\left(k_{0} \mu_{2} \Delta+k_{0} \rho \omega^{2}+a_{11}\right) v+a_{12}\left(k_{0} \Delta+a_{33}\right) \varphi-a_{13}\left(a_{31} v+a_{32} \varphi\right)=0,
$$


whence follows

$$
\begin{aligned}
& {\left[\left(k_{0} \Delta+a_{33}\right)\left(k_{0} \mu_{2} \Delta+k_{0} \rho \omega^{2}+a_{11}\right)-a_{13} a_{31}\right] v} \\
& +\left[a_{12} k_{0} \Delta+\left(a_{12} a_{33}-a_{13} a_{32}\right)\right] \varphi=0 .
\end{aligned}
$$

On the other hand, multiplying the first and second equations of (4.37) by $a_{23}$ and $a_{13}$, respectively, and subtracting we have

$$
\begin{aligned}
& {\left[a_{23} k_{0}\left(\mu_{2} \Delta+\rho \omega^{2}\right)+a_{11} a_{23}-a_{21} a_{13}\right] v} \\
& +\left[-a_{13} k_{0} \Delta+\left(a_{12} a_{23}-a_{22} a_{13}\right)\right] \varphi=0 .
\end{aligned}
$$

Finally, from (4.41) and (4.42) we obtain the first equation of (4.40). Similarly, from system (4.37) we get the second equation of (4.40). $\diamond$

We are now in a position to obtain the representation formula for a general solution of the system of homogeneous equations (4.4) by metaharmonic functions.

Obviously, the determinant of the system (4.36) is $\mu_{2} k_{0} \Lambda_{1}(\Delta)$, and hence, $v$ is a 3-metaharmonic function, i.e.

$$
\Lambda_{1}(\Delta) v=0
$$

where $\Lambda_{1}(\Delta)$ is defined by (4.12). Similarly, we have

$$
\Lambda_{1}(\Delta) \varphi=0, \quad \Lambda_{1}(\Delta) \theta=0 .
$$

In addition, there are the particular cases, when $v(\varphi$ and $\theta)$ is a metaharmonic or bi-metaharmonic function. For example, if $\beta=b_{1}=0$, then from the first equation of (4.36) we obtain

$$
\left(\Delta+\kappa_{11}^{2}\right) v(\mathbf{x})=0
$$

where $\kappa_{11}^{2}=\frac{\rho \omega^{2}}{\mu_{2}}$, i.e. $v$ is a metaharmonic function. 
On the other hand, if $d_{0}=0$, then on the basis of (4.40) we have $N_{1}(\Delta) v=0$ for $a_{13} \neq 0$ or $N_{2}(\Delta) v=0$ for $a_{12} \neq 0$, and therefore, $v$ is a bi-metaharmonic function.

In the follows we assume that $v$ is a 3-metaharmonic function, and hence, $d_{0} \neq 0$. The other cases (when $v$ is a metaharmonic or bi-metaharmonic function) are too simple to be considered.

Theorem 4.5. If $\varphi_{j}$ is a metaharmonic function

$$
\left(\Delta+\kappa_{j}^{2}\right) \varphi_{j}=0, \quad j=1,2,3,
$$

$\boldsymbol{\psi}=\left(\psi_{1}, \psi_{2}, \psi_{3}\right)$ is a metaharmonic vector function

$$
\left(\Delta+\kappa_{4}^{2}\right) \boldsymbol{\psi}=\mathbf{0}
$$

and

$$
\operatorname{div} \boldsymbol{\psi}=0
$$

then $(\mathbf{u}, \varphi, \theta)$ is a solution of (4.4), where

$$
\mathbf{u}=d_{j} \nabla \varphi_{j}+\boldsymbol{\psi}, \quad \varphi=e_{j} \varphi_{j}, \quad \theta=r_{j} \varphi_{j}
$$

and $\left(d_{j}, e_{j}, r_{j}\right)$ is a solution of the system

$$
\begin{aligned}
& \left(\mu_{2} \kappa_{j}^{2}-\rho \omega^{2}\right) d_{j}-b_{1} e_{j}+\beta r_{j}=0, \\
& -\nu_{1} \kappa_{j}^{2} d_{j}+\left(\alpha_{1} \kappa_{j}^{2}-\xi_{2}\right) e_{j}+\left(\kappa^{*} \tau_{j}^{2}-m\right) r_{j}=0, \\
& \beta_{1} \kappa_{j}^{2} d_{j}+\left(\zeta_{1} \kappa_{j}^{2}-m_{1}\right) e_{j}+\left(k \kappa_{j}^{2}-c_{1}\right) r_{j}=0
\end{aligned}
$$

with determinant equal to zero, $j=1,2,3$. 
Proof. Taking into account (4.44) to (4.48) by direct calculation we obtain

$$
\begin{aligned}
\left(\mu_{1} \Delta+\rho \omega^{2}\right) \mathbf{u}+\left(\lambda_{1}+\mu_{1}\right) \nabla \operatorname{div} \mathbf{u}+b_{1} \nabla \varphi-\beta \nabla \theta & \\
= & \sum_{j=1}^{3}\left[-\mu_{1}\left(\kappa_{j}^{2} d_{j} \nabla \varphi_{j}+\kappa_{4}^{2} \boldsymbol{\psi}\right)-\left(\lambda_{1}+\mu_{1}\right) \kappa_{j}^{2} d_{j} \nabla \varphi_{j}\right] \\
& +\sum_{j=1}^{3}\left[b_{1} e_{j} \nabla \varphi_{j}-\beta r_{j} \nabla \varphi_{j}+\rho \omega^{2} d_{j} \nabla \varphi_{j}+\rho \omega^{2} \boldsymbol{\psi}\right] \\
& =-\sum_{j=1}^{3}\left[\left(\mu_{2} \kappa_{j}^{2}-\rho \omega^{2}\right) d_{j}-b_{1} e_{j}+\beta r_{j}\right] \nabla \varphi_{j}=\mathbf{0} .
\end{aligned}
$$

Similarly, we have

$$
\begin{aligned}
& \left(\alpha_{1} \Delta+\xi_{2}\right) \varphi+\left(\tau^{*} \Delta+m\right) \theta-\nu_{1} \operatorname{div} \mathbf{u} \\
& =-\sum_{j=1}^{3}\left[-\nu_{1} \kappa_{j}^{2} d_{j}+\left(\alpha_{1} \kappa_{j}^{2}-\xi_{2}\right) e_{j}+\left(\kappa^{*} \kappa_{j}^{2}-m\right) r_{j}\right] \nabla \varphi_{j}=0, \\
& \left(k \Delta+c_{1}\right) \theta+\left(\zeta_{1} \Delta+m_{1}\right) \varphi+\beta_{1} \operatorname{div} \mathbf{u} \\
& =-\sum_{j=1}^{3}\left[\beta_{1} \kappa_{j}^{2} d_{j}+\left(\zeta_{1} \kappa_{j}^{2}-m_{1}\right) e_{j}+\left(k \kappa_{j}^{2}-c_{1}\right) r_{j}\right] \nabla \varphi_{j}=0 .
\end{aligned}
$$

Obviously, the determinant of the system (4.48) vanishes, i.e. $\mu_{2} k_{0} \Lambda_{1}\left(-\kappa_{j}^{2}\right)=0$. $\diamond$

Theorem 4.6. If $(\mathbf{u}, \varphi, \theta)$ is a solution of (4.6), then $\mathbf{u}, \varphi$ and $\theta$ are represented by (4.47), where $\varphi_{j}$ and $\boldsymbol{\psi}$ are solutions of equations (4.44) and (4.45), (4.46), respectively; $\left(d_{j}, e_{j}, r_{j}\right)$ is a solution of the system (4.48) $(j=1,2,3)$. 
Proof. We introduce the notations

$$
\varphi_{j}(\mathbf{x})=\frac{1}{p_{j}}\left[\prod_{l=1,2,3 ; l \neq j} \frac{1}{\left(\kappa_{l}^{2}-\kappa_{j}^{2}\right)}\left(\Delta+\kappa_{l}^{2}\right)\right] v(\mathbf{x}), \quad j=1,2,3
$$

and

$$
\boldsymbol{\psi}=\frac{1}{\kappa_{4}^{2}} \operatorname{curl} \operatorname{curl} \mathbf{u}
$$

where $p_{j}(j=1,2,3)$ is an arbitrary complex number and $p_{j} \neq 0$.

In view of (4.43) and (4.49) the function $\varphi_{j}$ is a solution of (4.44). Applying the operator curl to the first equation of (4.6) we obtain

$$
\left(\Delta+\kappa_{4}^{2}\right) \operatorname{curl} \mathbf{u}=\mathbf{0} .
$$

Taking into account (4.50) the vector function $\boldsymbol{\psi}$ is a solution of (4.45) and (4.46).

Obviously, from (4.49) we get

$$
v(\mathbf{x})=p_{j} \varphi_{j}(\mathbf{x})
$$

By virtue of (4.40) and (4.51) we have

$$
\begin{aligned}
\varphi(\mathbf{x}) & =\frac{1}{d_{0}} N_{1}(\Delta) v(\mathbf{x})=\frac{1}{d_{0}} \sum_{j=1}^{3} p_{j} N_{1}(\Delta) \varphi_{j}(\mathbf{x}) \\
& =\frac{1}{d_{0}} \sum_{j=1}^{3} p_{j} N_{1}\left(-\tau_{j}^{2}\right) \varphi_{j}(\mathbf{x}), \\
\theta(\mathbf{x}) & =\frac{1}{d_{0}} N_{2}(\Delta) v(\mathbf{x})=\frac{1}{d_{0}} \sum_{j=1}^{3} p_{j} N_{2}(\Delta) \varphi_{j}(\mathbf{x}) \\
& =\frac{1}{d_{0}} \sum_{j=1}^{3} p_{j} N_{2}\left(-\tau_{j}^{2}\right) \varphi_{j}(\mathbf{x}) .
\end{aligned}
$$


Therefore, $\varphi$ and $\theta$ are represented by (4.47), where

$$
e_{j}=\frac{1}{d_{0}} p_{j} N_{1}\left(-\kappa_{j}^{2}\right), \quad r_{j}=\frac{1}{d_{0}} p_{j} N_{2}\left(-\kappa_{j}^{2}\right)
$$

Using the identity

$$
\Delta \mathbf{u}=\nabla v-\operatorname{curl} \operatorname{curl} \mathbf{u}
$$

from the first equation of (4.6) we have

$$
\mathbf{u}=-\frac{1}{\rho \omega^{2}} \nabla\left(\mu_{2} v+b_{1} \varphi-\beta \theta\right)+\boldsymbol{\psi}
$$

Substituting the second and third equations of (4.47) and (4.51) in (4.52) we obtain

$$
\mathbf{u}=-\frac{1}{\rho \omega^{2}} \sum_{j=1}^{3}\left(\mu_{2} p_{j}+b_{1} e_{j}-\beta r_{j}\right) \nabla \varphi_{j}(\mathbf{x})+\boldsymbol{\psi}
$$

Hence, $\mathbf{u}$ is represented by (4.47), where

$$
d_{j}=-\frac{1}{\rho \omega^{2}}\left(\mu_{2} p_{j}+b_{1} e_{j}-\beta r_{j}\right)
$$

In addition, from the first equation of (4.47) and (4.51) it follows that $p_{j}=$ $-\kappa_{j}^{2} d_{j}$. By virtue of (4.53) we have

$$
d_{j}=\frac{1}{\rho \omega^{2}}\left(\mu_{2} \kappa_{j}^{2} d_{j}-b_{1} e_{j}+\beta r_{j}\right)
$$

whence $\left(d_{j}, e_{j}, r_{j}\right)$ is a solution of the first equation of (4.48). It is easy to verify that $\left(d_{j}, e_{j}, r_{j}\right)$ is a solution of the second and third equations of $(4.48)(j=$ $1,2,3)$.

Thus, the general solution of the system of homogeneous equations (4.6) in terms of six metaharmonic functions $\varphi_{j}$ and $\psi_{j}(j=1,2,3)$ is obtained. $\diamond$

By theorems 4.5 and 4.6 the completeness of general solution of system (4.6) is proved. 


\subsection{Green's formulas. Integral representation of solution}

In this section, first, we establish the Green's formulas in the linear theory of thermoviscoelasticity for Kelvin-Voigt materials with voids, then we obtain the integral representation of regular vector (representation of Somigliana-type) and a regular solution of the system (4.4).

In the sequel we use the matrix differential operators $\tilde{\mathcal{A}}\left(\mathbf{D}_{\mathbf{x}}\right), \mathbf{R}\left(\mathbf{D}_{\mathbf{x}}, \mathbf{n}\right)$ and $\tilde{\mathbf{R}}\left(\mathbf{D}_{\mathbf{x}}, \mathbf{n}\right)$, where

1)

$$
\tilde{\mathcal{A}}\left(\mathbf{D}_{\mathbf{x}}\right)=\mathcal{A}^{\top}\left(-\mathbf{D}_{\mathbf{x}}\right)
$$

2)

$$
\begin{aligned}
& \mathbf{R}\left(\mathbf{D}_{\mathbf{x}}, \mathbf{n}\right)=\left(R_{p q}\left(\mathbf{D}_{\mathbf{x}}, \mathbf{n}\right)\right)_{5 \times 5} \\
& R_{l j}\left(\mathbf{D}_{\mathbf{x}}, \mathbf{n}\right)=\mu_{1} \delta_{l j} \frac{\partial}{\partial \mathbf{n}}+\mu_{1} n_{j} \frac{\partial}{\partial x_{l}}+\lambda_{1} n_{l} \frac{\partial}{\partial x_{j}} \\
& R_{l 4}\left(\mathbf{D}_{\mathbf{x}}, \mathbf{n}\right)=b_{1} n_{l}, \quad R_{l 5}\left(\mathbf{D}_{\mathbf{x}}, \mathbf{n}\right)=-\beta n_{l}, \\
& R_{4 l}\left(\mathbf{D}_{\mathbf{x}}, \mathbf{n}\right)=R_{5 l}\left(\mathbf{D}_{\mathbf{x}}, \mathbf{n}\right)=0, \quad R_{44}\left(\mathbf{D}_{\mathbf{x}}, \mathbf{n}\right)=\alpha_{1} \frac{\partial}{\partial \mathbf{n}}, \\
& R_{45}\left(\mathbf{D}_{\mathbf{x}}, \mathbf{n}\right)=\tau^{*} \frac{\partial}{\partial \mathbf{n}}, \quad R_{54}\left(\mathbf{D}_{\mathbf{x}}, \mathbf{n}\right)=\zeta_{1} \frac{\partial}{\partial \mathbf{n}}, \\
& R_{55}\left(\mathbf{D}_{\mathbf{x}}, \mathbf{n}\right)=k \frac{\partial}{\partial \mathbf{n}}, \quad l, j=1,2,3
\end{aligned}
$$


3)

$$
\begin{aligned}
& \tilde{\mathbf{R}}\left(\mathbf{D}_{\mathbf{x}}, \mathbf{n}\right)=\left(\tilde{\mathbf{R}}_{p q}\left(\mathbf{D}_{\mathbf{x}}, \mathbf{n}\right)\right)_{5 \times 5}, \quad \tilde{\mathbf{R}}_{l j}\left(\mathbf{D}_{\mathbf{x}}, \mathbf{n}\right)=\mathbf{R}_{l j}\left(\mathbf{D}_{\mathbf{x}}, \mathbf{n}\right), \\
& \tilde{\mathbf{R}}_{l 4}\left(\mathbf{D}_{\mathbf{x}}, \mathbf{n}\right)=\nu_{1} n_{l}, \quad \tilde{\mathbf{R}}_{l 5}\left(\mathbf{D}_{\mathbf{x}}, \mathbf{n}\right)=-\beta_{1} n_{l}, \\
& \tilde{\mathbf{R}}_{p q}\left(\mathbf{D}_{\mathbf{x}}, \mathbf{n}\right)=\mathbf{R}_{q p}\left(\mathbf{D}_{\mathbf{x}}, \mathbf{n}\right), \quad \tilde{\mathbf{R}}_{4 l}\left(\mathbf{D}_{\mathbf{x}}, \mathbf{n}\right)=\tilde{\mathbf{R}}_{5 l}\left(\mathbf{D}_{\mathbf{x}}, \mathbf{n}\right)=0, \\
& l, j=1,2,3, \quad p, q=4,5 .
\end{aligned}
$$

Obviously, the matrix differential operator $\tilde{\mathcal{A}}\left(\mathbf{D}_{\mathbf{x}}\right)$ is the associate operator of $\mathcal{A}\left(\mathbf{D}_{\mathbf{x}}\right)$. It is easy to verify that the operator $\tilde{\mathcal{A}}\left(\mathbf{D}_{\mathbf{x}}\right)$ may be obtained from the operator $\mathcal{A}\left(\mathbf{D}_{\mathbf{x}}\right)$ by replacing $b_{1}, \beta, \tau^{*}, m$ by $\nu_{1}, \beta_{1}, \zeta_{1}, m_{1}$, respectively, and vice versa (see (4.7)). On the basis of (4.54) the associated homogeneous system of equations $\tilde{\mathcal{A}}\left(\mathbf{D}_{\mathbf{x}}\right) \mathbf{V}(\mathbf{x})=\mathbf{0}$ has the following form

$$
\begin{aligned}
& \left(\mu_{1} \Delta+\rho \omega^{2}\right) \mathbf{u}+\left(\lambda_{1}+\mu_{1}\right) \nabla \operatorname{div} \mathbf{u}+\nu_{1} \nabla \varphi-\beta_{1} \nabla \theta=\mathbf{0}, \\
& \left(\alpha_{1} \Delta+\xi_{2}\right) \varphi+\left(\zeta_{1} \Delta+m_{1}\right) \theta-b_{1} \operatorname{div} \mathbf{u}=0, \\
& \left(k \Delta+c_{1}\right) \theta+\left(\tau^{*} \Delta+m\right) \varphi+\beta \operatorname{div} \mathbf{u}=0 .
\end{aligned}
$$

Similarly, operator $\tilde{\mathbf{R}}\left(\mathbf{D}_{\mathbf{x}}, \mathbf{n}\right)$ may be obtained from the operator $\mathbf{R}\left(\mathbf{D}_{\mathbf{x}}, \mathbf{n}\right)$ by replacing $b_{1}, \beta, \tau^{*}$ by $\nu_{1}, \beta_{1}, \zeta_{1}$, respectively, and vice versa. In addition, (on the basis of (4.54) the fundamental matrix $\tilde{\boldsymbol{\Theta}}(\mathbf{x})$ of operator $\tilde{\mathcal{A}}\left(\mathbf{D}_{\mathbf{x}}\right)$ satisfies the following condition

$$
\tilde{\Theta}(\mathbf{x})=\Theta^{\top}(-\mathbf{x})
$$

where $\boldsymbol{\Theta}(\mathbf{x})$ is the fundamental solution of the system (4.6) (the fundamental matrix of the operator $\left.\mathcal{A}\left(\mathbf{D}_{\mathbf{x}}\right)\right)$.

Let $\mathbf{V}=(\mathbf{u}, \varphi, \theta)=\left(V_{1}, V_{2}, \cdots, V_{5}\right)$, the vector $\tilde{\mathbf{V}}_{j}$ is the $j$-th column of the $\operatorname{matrix} \tilde{\mathbf{V}}=\left(\tilde{V}_{l j}\right)_{5 \times 5}, \tilde{\mathbf{u}}_{j}=\left(\tilde{V}_{1 j}, \tilde{V}_{2 j}, \tilde{V}_{3 j}\right)^{\top}, \tilde{\varphi}_{j}=\tilde{V}_{4 j}, \tilde{\theta}_{j}=\tilde{V}_{5 j}, j=1,2, \cdots, 5$. 
Theorem 4.7. If $V$ and $\tilde{V}_{j}(j=1,2, \cdots, 5)$ are regular vectors in $\Omega^{+}$, then

$$
\begin{aligned}
& \int_{\Omega^{+}}\left\{\left[\tilde{\mathbf{A}}\left(\mathbf{D}_{\mathbf{y}}\right) \tilde{\mathbf{V}}(\mathbf{y})\right]^{\top} \mathbf{V}(\mathbf{y})-[\tilde{\mathbf{V}}(\mathbf{y})]^{\top} \mathbf{A}\left(\mathbf{D}_{\mathbf{y}}\right) \mathbf{V}(\mathbf{y})\right\} d \mathbf{y} \\
& =\int_{S}\left\{\left[\tilde{\mathbf{R}}\left(\mathbf{D}_{\mathbf{z}}, \mathbf{n}\right) \tilde{\mathbf{V}}(\mathbf{z})\right]^{\top} \mathbf{V}(\mathbf{z})-[\tilde{\mathbf{U}}(\mathbf{z})]^{\top} \mathbf{R}\left(\mathbf{D}_{\mathbf{z}}, \mathbf{n}\right) \tilde{\mathbf{V}}(\mathbf{z})\right\} d_{\mathbf{z}} S .
\end{aligned}
$$

Proof. We consider the difference $(\tilde{\mathcal{A}} \tilde{\mathbf{V}})^{\top} \mathbf{V}-(\tilde{\mathbf{V}})^{\top} \mathcal{A V}$. The $j$-th $(j=1,2, \cdots, 5)$ component of this vector may be written in the form

$$
\begin{aligned}
& {\left[(\tilde{\mathcal{A}} \tilde{\mathbf{V}})^{\top} \mathbf{V}-\tilde{\mathbf{V}}^{\top} \mathcal{A} \mathbf{V}\right]_{j}=\left(\mathbf{B} \tilde{\mathbf{u}}_{j}+\rho \omega^{2} \tilde{\mathbf{u}}_{j}+\nu_{1} \nabla \tilde{\varphi}_{j}-\beta_{1} \nabla \tilde{\theta}_{j}\right) \mathbf{u}} \\
& +\left(\alpha_{1} \Delta \tilde{\varphi}_{j}+\xi_{2} \tilde{\varphi}_{j}+\zeta_{1} \Delta \tilde{\theta}_{j}+m_{1} \tilde{\theta}_{j}-b_{1} \operatorname{div} \tilde{\mathbf{u}}_{j}\right) \varphi \\
& +\left(k \Delta \tilde{\theta}_{j}+c_{1} \tilde{\theta}_{j}+\tau^{*} \Delta \tilde{\varphi}_{j}+m \tilde{\varphi}_{j}+\beta \operatorname{div} \tilde{\mathbf{u}}_{j}\right) \theta \\
& -\tilde{\mathbf{u}}_{j}\left(\mathbf{B u}+\rho \omega^{2} \mathbf{u}+b_{1} \nabla \varphi-\beta \nabla \theta\right) \\
& -\tilde{\theta}_{j}\left(k \Delta \theta+\tilde{\varphi}_{j}\left(\alpha_{1} \Delta \varphi+\xi_{2} \varphi+\tau^{*} \Delta \theta+m \theta-\nu_{1} \operatorname{div} \mathbf{u}\right)\right. \\
& =\left(\mathbf{B} \tilde{\mathbf{u}}_{j} \mathbf{u}-\tilde{\mathbf{u}}_{j} \mathbf{B u}\right)+\alpha_{1}\left(\Delta \tilde{\varphi}_{j} \varphi-\tilde{\varphi}_{j} \operatorname{div} \mathbf{u}\right) \\
& +k\left(\Delta \tilde{\theta}_{j} \theta-\tilde{\theta}_{j} \Delta \theta\right)+\tau_{1}^{*}\left(\Delta \tilde{\varphi}_{j} \theta-\tilde{\varphi}_{j} \Delta \theta\right)+\nu_{1}\left(\nabla \tilde{\varphi}_{j} \mathbf{u}+\tilde{\varphi}_{j} \operatorname{div} \mathbf{u}\right) \\
& +\beta\left(\operatorname{div} \tilde{\mathbf{u}}_{j} \theta+\tilde{\theta}_{j} \Delta \varphi\right) \\
& -\beta_{1}\left(\nabla \tilde{\theta}_{j} \mathbf{u}+\tilde{\theta}_{j} \operatorname{div} \mathbf{u}\right)-b_{1}\left(\operatorname{div} \tilde{\mathbf{u}}_{j} \varphi+\tilde{\mathbf{u}}_{j} \nabla \varphi\right) \\
& \left.+\tilde{\mathbf{u}}_{j}\right)
\end{aligned}
$$


Integrating over $\Omega^{+}$, using the identities (see, e.g., Kupradze et al. [83], Ch. III)

$$
\begin{aligned}
& \int_{\Omega^{+}}\left(\mathbf{B} \tilde{\mathbf{u}}_{j} \mathbf{u}-\tilde{\mathbf{u}}_{j} \mathbf{B u}\right) d \mathbf{y}=\int_{S}\left(\mathbf{T} \tilde{\mathbf{u}}_{j} \mathbf{u}-\tilde{\mathbf{u}}_{j} \mathbf{T u}\right) d_{\mathbf{z}} S \\
& \int_{\Omega^{+}}\left(\Delta \tilde{\varphi}_{j} \varphi-\tilde{\varphi}_{j} \Delta \varphi\right) d \mathbf{y}=\int_{S}\left(\frac{\partial \tilde{\varphi}_{j}}{\partial \mathbf{n}} \varphi-\tilde{\varphi}_{j} \frac{\partial \varphi}{\partial \mathbf{n}}\right) d_{\mathbf{z}} S, \\
& \int_{\Omega^{+}}\left(\nabla \tilde{\varphi}_{j} \mathbf{u}+\tilde{\varphi}_{j} \operatorname{div} \mathbf{u}\right) d \mathbf{y}=\int_{S} \tilde{\varphi}_{j} \mathbf{u} \mathbf{n} d_{\mathbf{z}} S \\
& \int_{\Omega^{+}}\left(\operatorname{div} \tilde{\mathbf{u}}_{j} \varphi+\tilde{\mathbf{u}}_{j} \nabla \varphi\right) d \mathbf{y}=\int_{S} \tilde{\mathbf{u}}_{j} \varphi \mathbf{n} d_{\mathbf{z}} S
\end{aligned}
$$

from (4.60) it follows that

$$
\begin{aligned}
& \int_{\Omega^{+}}\left[(\tilde{\mathbf{A}} \tilde{\mathbf{U}})^{\top} \mathbf{U}-\tilde{\mathbf{U}}^{\top} \mathbf{A} \mathbf{U}\right]_{j} d \mathbf{y} \\
& =\int_{S}\left[\left(\mathbf{T} \tilde{\mathbf{u}}_{j} \mathbf{u}-\tilde{\mathbf{u}}_{j} \mathbf{T u}\right)+\alpha_{1}\left(\frac{\partial \tilde{\varphi}_{j}}{\partial \mathbf{n}} \varphi-\tilde{\varphi}_{j} \frac{\partial \varphi}{\partial \mathbf{n}}\right)\right] d_{\mathbf{z}} S \\
& +\int_{S}\left[\zeta_{1}\left(\frac{\partial \tilde{\theta}_{j}}{\partial \mathbf{n}} \varphi-\tilde{\theta}_{j} \frac{\partial \varphi}{\partial \mathbf{n}}\right)+k\left(\frac{\partial \tilde{\theta}_{j}}{\partial \mathbf{n}} \theta-\tilde{\theta}_{j} \frac{\partial \theta}{\partial \mathbf{n}}\right)+\tau^{*}\left(\frac{\partial \tilde{\varphi}_{j}}{\partial \mathbf{n}} \theta-\tilde{\varphi}_{j} \frac{\partial \theta}{\partial \mathbf{n}}\right)\right] d_{\mathbf{z}} S \\
& +\int_{S}\left[\left(\nu_{1} \tilde{\varphi}_{j}-\beta_{1} \tilde{\theta}_{j}\right) \mathbf{u}-b_{1} \tilde{\mathbf{u}}_{j} \varphi+\beta \tilde{\mathbf{u}}_{j} \theta\right] \mathbf{n} d_{\mathbf{z}} S
\end{aligned}
$$




$$
\begin{aligned}
& =\int_{S}\left[\left(\mathbf{T} \tilde{\mathbf{u}}_{j}+\nu_{1} \tilde{\varphi}_{j} \mathbf{n}-\beta_{1} \tilde{\theta}_{j} \mathbf{n}\right) \mathbf{u}+\left(\alpha_{1} \frac{\partial \tilde{\varphi}_{j}}{\partial \mathbf{n}}+\zeta_{1} \frac{\partial \tilde{\theta}_{j}}{\partial \mathbf{n}}\right) \varphi+\left(\tau^{*} \frac{\partial \tilde{\varphi}_{j}}{\partial \mathbf{n}}+k \frac{\partial \tilde{\theta}_{j}}{\partial \mathbf{n}}\right) \theta\right] d_{\mathbf{z}} S \\
& -\int_{S}\left[\tilde{\mathbf{u}}_{j}\left(\mathbf{T u}+b_{1} \varphi \mathbf{n}-\beta \theta \mathbf{n}\right)+\tilde{\varphi}_{j}\left(\alpha_{1} \frac{\partial \varphi}{\partial \mathbf{n}}+\tau^{*} \frac{\partial \theta}{\partial \mathbf{n}}\right)+\tilde{\theta}_{j}\left(\zeta_{1} \frac{\partial \varphi}{\partial \mathbf{n}}+k \frac{\partial \theta}{\partial \mathbf{n}}\right)\right] d_{\mathbf{z}} S \\
& =\int_{S}\left\{\left[\tilde{\mathbf{R}}\left(\mathbf{D}_{\mathbf{z}}, \mathbf{n}\right) \tilde{\mathbf{V}}(\mathbf{z})\right]^{\top} \mathbf{V}(\mathbf{z})-[\tilde{\mathbf{V}}(\mathbf{z})]^{\top} \mathbf{R}\left(\mathbf{D}_{\mathbf{z}}, \mathbf{n}\right) \mathbf{V}(\mathbf{z})\right\}_{j} d_{\mathbf{z}} S
\end{aligned}
$$

and formula (4.59) is thereby proved. $\diamond$

On the basis of theorem 4.7 and the condition (1.1) we obtain the following result.

Theorem 4.8. If $V$ and $\tilde{V}_{j}(j=1,2,3,4)$ are regular vectors in $\Omega^{-}$, then

$$
\begin{aligned}
& \int_{\Omega^{-}}\left\{\left[\tilde{\mathcal{A}}\left(\mathbf{D}_{\mathbf{y}}\right) \tilde{\mathbf{V}}(\mathbf{y})\right]^{\top} \mathbf{V}(\mathbf{y})-[\tilde{\mathbf{V}}(\mathbf{y})]^{\top} \mathcal{A}\left(\mathbf{D}_{\mathbf{y}}\right) \mathbf{V}(\mathbf{y})\right\} d \mathbf{y} \\
& =-\int_{S}\left\{\left[\tilde{\mathbf{R}}\left(\mathbf{D}_{\mathbf{z}}, \mathbf{n}\right) \tilde{\mathbf{V}}(\mathbf{z})\right]^{\top} \mathbf{V}(\mathbf{z})-[\tilde{\mathbf{V}}(\mathbf{z})]^{\top} \mathbf{R}\left(\mathbf{D}_{\mathbf{z}}, \mathbf{n}\right) \tilde{\mathbf{V}}(\mathbf{z})\right\} d_{\mathbf{z}} S .
\end{aligned}
$$

The identities (4.59) and (4.61) are the Green's formulas in the linear theory of thermoviscoelastic materials with voids for domains $\Omega^{+}$and $\Omega^{-}$, respectively.

Keeping in mind (4.57) and (4.58) from (4.59) and (4.61) we obtain the formulas of integral representation of regular vector and regular solution (representation of Somigliana-type) for the domains $\Omega^{+}$and $\Omega^{-}$.

Theorem 4.9. If $V$ is a regular vector in $\Omega^{+}$, then

$$
\begin{aligned}
\mathbf{V}(\mathbf{x})= & \int_{S}\left\{\left[\tilde{\mathbf{R}}\left(\mathbf{D}_{\mathbf{z}}, \mathbf{n}\right) \boldsymbol{\Theta}^{\top}(\mathbf{x}-\mathbf{z})\right]^{\top} \mathbf{V}(\mathbf{z})-\boldsymbol{\Theta}(\mathbf{x}-\mathbf{z}) \mathbf{R}\left(\mathbf{D}_{\mathbf{z}}, \mathbf{n}\right) \mathbf{V}(\mathbf{z})\right\} d_{\mathbf{z}} S \\
& +\int_{\Omega^{+}} \boldsymbol{\Theta}(\mathbf{x}-\mathbf{y}) \mathcal{A}\left(\mathbf{D}_{\mathbf{y}}\right) \mathbf{V}(\mathbf{y}) d \mathbf{y} \quad \text { for } \quad \mathbf{x} \in \Omega^{+} .
\end{aligned}
$$


Theorem 4.10. If $V$ is a regular vector in $\Omega^{-}$, then

$$
\begin{aligned}
\mathbf{V}(\mathbf{x})= & -\int_{S}\left\{\left[\tilde{\mathbf{R}}\left(\mathbf{D}_{\mathbf{z}}, \mathbf{n}\right) \boldsymbol{\Theta}^{\top}(\mathbf{x}-\mathbf{z})\right]^{\top} \mathbf{V}(\mathbf{z})-\boldsymbol{\Theta}(\mathbf{x}-\mathbf{z}) \mathbf{R}\left(\mathbf{D}_{\mathbf{z}}, \mathbf{n}\right) \mathbf{V}(\mathbf{z})\right\} d_{\mathbf{z}} S \\
& +\int_{\Omega^{-}} \boldsymbol{\Theta}(\mathbf{x}-\mathbf{y}) \mathcal{A}\left(\mathbf{D}_{\mathbf{y}}\right) \mathbf{V}(\mathbf{y}) d \mathbf{y} \quad \text { for } \quad \mathbf{x} \in \Omega^{-} .
\end{aligned}
$$

Obviously, on the basis of (4.62) and (4.63) we have the following results.

Corollary 4.3. If $V$ is a regular solution of (4.4) in $\Omega^{+}$, then

$$
\mathbf{V}(\mathbf{x})=\int_{S}\left\{\left[\tilde{\mathbf{R}}\left(\mathbf{D}_{\mathbf{z}}, \mathbf{n}\right) \boldsymbol{\Theta}^{\top}(\mathbf{x}-\mathbf{z})\right]^{\top} \mathbf{V}(\mathbf{z})-\boldsymbol{\Theta}(\mathbf{x}-\mathbf{z}) \mathbf{R}\left(\mathbf{D}_{\mathbf{z}}, \mathbf{n}\right) \mathbf{V}(\mathbf{z})\right\} d_{\mathbf{z}} S
$$

for $\mathbf{x} \in \Omega^{+}$.

Corollary 4.4. If $V$ is a regular solution of (4.4) in $\Omega^{-}$, then

$$
\mathbf{V}(\mathbf{x})=-\int_{S}\left\{\left[\tilde{\mathbf{R}}\left(\mathbf{D}_{\mathbf{z}}, \mathbf{n}\right) \boldsymbol{\Theta}^{\top}(\mathbf{x}-\mathbf{z})\right]^{\top} \mathbf{V}(\mathbf{z})-\boldsymbol{\Theta}(\mathbf{x}-\mathbf{z}) \mathbf{R}\left(\mathbf{D}_{\mathbf{z}}, \mathbf{n}\right) \mathbf{V}(\mathbf{z})\right\} d_{\mathbf{z}} S
$$

for $\mathbf{x} \in \Omega^{-}$.

Keeping in mind the conditions (1.1) and (4.10) from (4.64) we obtain

$$
\begin{aligned}
& V_{j}(x)=e^{-\kappa_{0}|\mathbf{x}|} O\left(|\mathbf{x}|^{-1}\right), \\
& V_{j, l}(x)=e^{-\kappa_{0}|\mathbf{x}|} O\left(|\mathbf{x}|^{-1}\right) \quad \text { for } \quad|\mathbf{x}| \gg 1,
\end{aligned}
$$

where $\kappa_{0}=\min \left\{\operatorname{Im} \kappa_{1}, \operatorname{Im} \kappa_{2}, \operatorname{Im} \kappa_{3}, \operatorname{Im} \kappa_{4}\right\}>0, l=1,2,3, j=1,2,3,4$. 


\section{Chapter 5}

\section{Boundary value problems in the theory of thermoviscoelasticity}

\section{for materials with voids}

\subsection{Basic boundary value problems}

In the sequel we use the matrix differential operators

1)

$$
\begin{aligned}
& \mathbf{R}^{(1)}\left(\mathbf{D}_{\mathbf{x}}, \mathbf{n}\right)=\left(R_{p q}^{(1)}\left(\mathbf{D}_{\mathbf{x}}, \mathbf{n}\right)\right)_{3 \times 5} \\
& R_{l j}^{(1)}\left(\mathbf{D}_{\mathbf{x}}, \mathbf{n}\right)=\mu_{1} \delta_{l j} \frac{\partial}{\partial \mathbf{n}}+\mu_{1} n_{j} \frac{\partial}{\partial x_{l}}+\lambda_{1} n_{l} \frac{\partial}{\partial x_{j}} \\
& R_{l 4}^{(1)}\left(\mathbf{D}_{\mathbf{x}}, \mathbf{n}\right)=b_{1} n_{l}, \quad R_{l 5}^{(1)}\left(\mathbf{D}_{\mathbf{x}}, \mathbf{n}\right)=-\beta n_{l} ;
\end{aligned}
$$

2)

$$
\begin{aligned}
& \mathbf{R}^{(2)}\left(\mathbf{D}_{\mathbf{x}}, \mathbf{n}\right)=\left(R_{p q}^{(2)}\left(\mathbf{D}_{\mathbf{x}}, \mathbf{n}\right)\right)_{1 \times 5}, \quad R_{1 l}^{(2)}\left(\mathbf{D}_{\mathbf{x}}, \mathbf{n}\right)=0 \\
& R_{14}^{(2)}\left(\mathbf{D}_{\mathbf{x}}, \mathbf{n}\right)=\alpha_{1} \frac{\partial}{\partial \mathbf{n}}, \quad R_{15}^{(2)}\left(\mathbf{D}_{\mathbf{x}}, \mathbf{n}\right)=\tau^{*} \frac{\partial}{\partial \mathbf{n}}
\end{aligned}
$$


3)

$$
\begin{aligned}
& \mathbf{R}^{(3)}\left(\mathbf{D}_{\mathbf{x}}, \mathbf{n}\right)=\left(R_{p q}^{(3)}\left(\mathbf{D}_{\mathbf{x}}, \mathbf{n}\right)\right)_{1 \times 5}, \quad R_{1 j}^{(3)}\left(\mathbf{D}_{\mathbf{x}}, \mathbf{n}\right)=0 \\
& R_{14}^{(3)}\left(\mathbf{D}_{\mathbf{x}}, \mathbf{n}\right)=\zeta_{1} \frac{\partial}{\partial \mathbf{n}}, \quad R_{14}^{(3)}\left(\mathbf{D}_{\mathbf{x}}, \mathbf{n}\right)=k \frac{\partial}{\partial \mathbf{n}}
\end{aligned}
$$

where $l, j=1,2,3$.

Obviously, we can rewrite the matrix differential operator $\mathbf{R}\left(\mathbf{D}_{\mathbf{x}}, \mathbf{n}\right)$ (see (4.55)) in the form

$$
\begin{aligned}
& \mathbf{R}\left(\mathbf{D}_{\mathbf{x}}, \mathbf{n}\right)=\left(R_{p q}\left(\mathbf{D}_{\mathbf{x}}, \mathbf{n}\right)\right)_{5 \times 5}, \quad R_{l q}\left(\mathbf{D}_{\mathbf{x}}, \mathbf{n}\right)=R_{l q}^{(1)}\left(\mathbf{D}_{\mathbf{x}}, \mathbf{n}\right), \\
& R_{4 q}\left(\mathbf{D}_{\mathbf{x}}, \mathbf{n}\right)=R_{1 q}^{(2)}\left(\mathbf{D}_{\mathbf{x}}, \mathbf{n}\right), \quad R_{5 q}\left(\mathbf{D}_{\mathbf{x}}, \mathbf{n}\right)=R_{1 q}^{(3)}\left(\mathbf{D}_{\mathbf{x}}, \mathbf{n}\right),
\end{aligned}
$$

where $l=1,2,3, q=1,2, \cdots, 5$.

The basic internal and external BVPs of steady vibrations in the theory of thermoviscoelasticity for Kelvin-Voigt materials with voids are formulated as follows.

Find a regular (classical) solution to system (4.8) for $\mathbf{x} \in \Omega^{+}$satisfying the boundary condition

$$
\lim _{\Omega^{+} \ni \mathbf{x} \rightarrow \mathbf{z} \in S} \mathbf{V}(\mathbf{x}) \equiv\{\mathbf{V}(\mathbf{z})\}^{+}=\mathbf{f}(\mathbf{z})
$$

in the Problem $(I I I)_{\mathbf{F}, \mathbf{f}}^{+}$, and

$$
\lim _{\Omega^{+} \ni \mathbf{x} \rightarrow \mathbf{z} \in S} \mathbf{R}\left(\mathbf{D}_{\mathbf{x}}, \mathbf{n}(\mathbf{z})\right) \mathbf{V}(\mathbf{x}) \equiv\left\{\mathbf{R}\left(\mathbf{D}_{\mathbf{z}}, \mathbf{n}(\mathbf{z})\right) \mathbf{V}(\mathbf{z})\right\}^{+}=\mathbf{f}(\mathbf{z})
$$

in the Problem $(I V)_{\mathbf{F}, \mathbf{f}}^{+}$, where $\mathbf{F}$ and $\mathbf{f}$ are the known five-component vector functions.

Find a regular (classical) solution to system (4.8) for $\mathbf{x} \in \Omega^{-}$satisfying the boundary condition

$$
\lim _{\Omega^{-} \ni \mathbf{x} \rightarrow \mathbf{z} \in S} \mathbf{V}(\mathbf{x}) \equiv\{\mathbf{V}(\mathbf{z})\}^{-}=\mathbf{f}(\mathbf{z})
$$


in the Problem $(I I I)_{\mathbf{F}, \mathbf{f}}^{-}$, and

$$
\lim _{\Omega^{-} \ni \mathbf{x} \rightarrow \mathbf{z} \in S} \mathbf{R}\left(\mathbf{D}_{\mathbf{x}}, \mathbf{n}(\mathbf{z})\right) \mathbf{V}(\mathbf{x}) \equiv\left\{\mathbf{R}\left(\mathbf{D}_{\mathbf{z}}, \mathbf{n}(\mathbf{z})\right) \mathbf{V}(\mathbf{z})\right\}^{-}=\mathbf{f}(\mathbf{z})
$$

in the Problem $(I V)_{\mathbf{F}, \mathbf{f}}^{-}$, where $\mathbf{F}$ and $\mathbf{f}$ are the known five-component vector functions, and $\operatorname{supp} \mathbf{F}$ is a finite domain in $\Omega^{-}$.

\subsection{Uniqueness theorems}

In this section we prove uniqueness of regular solutions of BVPs $(K)_{\mathbf{F}, \mathbf{f}}^{+}$and $(K)_{\mathbf{F}, \mathbf{f}}^{-}$, where $K=I I I, I V$.

Theorem 5.1. If condition (4.10) is satisfied, then the internal BVP $(K)_{\mathbf{F}, \mathbf{f}}^{+}$ admits at most one regular solution, where $K=I I I, I V$.

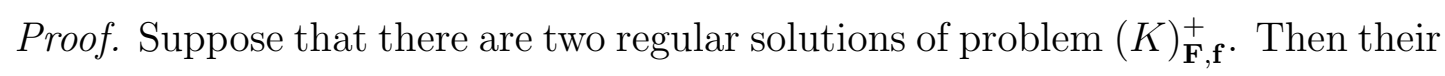
difference $\mathbf{V}$ corresponds to zero data $(\mathbf{F}=\mathbf{f}=\mathbf{0})$, i.e. $\mathbf{V}$ is a regular solution of problem $(K)_{\mathbf{0}, \mathbf{0}}^{+}$, i.e. the vector $\mathbf{V}=(\mathbf{u}, \varphi, \theta)$ is a solution of the system of homogeneous equations (4.6) satisfying the homogeneous boundary condition

$$
\{\mathbf{U}(\mathbf{z})\}^{+}=\mathbf{0}
$$

in the case $K=I I I$, and

$$
\left\{\mathbf{R}\left(\mathbf{D}_{\mathbf{z}}, \mathbf{n}(\mathbf{z})\right) \mathbf{U}(\mathbf{z})\right\}^{+}=\mathbf{0}
$$

in the case $K=I V$. Obviously, we can rewrite the boundary condition (5.6) in the form

$$
\left\{\mathbf{R}^{(j)}\left(\mathbf{D}_{\mathbf{z}}, \mathbf{n}(\mathbf{z})\right) \mathbf{V}(\mathbf{z})\right\}^{+}=\mathbf{0}, \quad j=1,2,3 .
$$

On account of (4.6) from Green's formula of the classical theory of elasticity 
(see Section 3.2)

$$
\int_{\Omega^{+}}\left[\mathbf{B}\left(\mathbf{D}_{\mathbf{x}}\right) \mathbf{u} \cdot \mathbf{u}+W\left(\mathbf{u}, \lambda_{1}, \mu_{1}\right)\right] d \mathbf{x}=\int_{S} \mathbf{T u} \cdot \mathbf{u} d_{\mathbf{z}} S
$$

and identities

$$
\begin{aligned}
& \int_{\Omega^{+}}(\nabla \varphi \cdot \mathbf{u}+\varphi \operatorname{div} \overline{\mathbf{u}}) d \mathbf{x}=\int_{S} \varphi \mathbf{n} \cdot \mathbf{u} d_{\mathbf{z}} S, \\
& \int_{\Omega^{+}}(\nabla \theta \cdot \mathbf{u}+\theta \operatorname{div} \overline{\mathbf{u}}) d \mathbf{x}=\int_{S} \theta \mathbf{n} \cdot \mathbf{u} d_{\mathbf{z}} S,
\end{aligned}
$$

it follows that

$$
\begin{aligned}
& \int_{\Omega^{+}}\left\{\left[\mathbf{B}\left(\mathbf{D}_{\mathbf{x}}\right) \mathbf{u}+b_{1} \nabla \varphi-\beta \nabla \theta+\rho \omega^{2}|\mathbf{u}|^{2}\right] \cdot \mathbf{u}+W^{(1)}(\mathbf{V})\right\} d \mathbf{x} \\
& =\int_{S} \mathbf{R}^{(1)}\left(\mathbf{D}_{\mathbf{z}}, \mathbf{n}\right) \mathbf{U}(\mathbf{z}) \cdot \mathbf{u}(\mathbf{z}) d_{\mathbf{z}} S
\end{aligned}
$$

where $W\left(\mathbf{u}, \lambda_{1}, \mu_{1}\right)$ are given by (3.7) and

$$
W^{(1)}(\mathbf{V})=W\left(\mathbf{u}, \lambda_{1}, \mu_{1}\right)-\rho \omega^{2}|\mathbf{u}|^{2}+\left(b_{1} \varphi-\beta \theta\right) \operatorname{div} \overline{\mathbf{u}} .
$$

By virtue of the first equation of (4.6), the boundary condition (5.5) in the case $K=I I I$ and (5.7) in the case $K=I V$, from (5.8) we get

$$
\int_{\Omega^{+}} W^{(1)}(\mathbf{V}) d \mathbf{x}=0
$$


On the other hand, on the basis of identities

$$
\begin{gathered}
\int_{\Omega^{+}}\left[\Delta \varphi \bar{\varphi}+|\nabla \varphi|^{2}\right] d \mathbf{x}=\int_{S} \frac{\partial \varphi}{\partial \mathbf{n}} \bar{\varphi} d_{\mathbf{z}} S, \\
\int_{\Omega^{+}}[\Delta \theta \bar{\varphi}+\nabla \theta \cdot \nabla \varphi] d \mathbf{x}=\int_{S} \frac{\partial \theta}{\partial \mathbf{n}} \bar{\varphi} d_{\mathbf{z}} S,
\end{gathered}
$$

we have

$$
\begin{aligned}
& \int_{\Omega^{+}}\left\{\left[\left(\alpha_{1} \Delta+\xi_{2}\right) \varphi+\left(\tau^{*} \Delta+m\right) \theta-\nu_{1} \operatorname{div} \mathbf{u}\right] \bar{\varphi}+W^{(2)}(\mathbf{U})\right\} d \mathbf{x} \\
& =\int_{S} \mathbf{R}^{(2)}\left(\mathbf{D}_{\mathbf{z}}, \mathbf{n}\right) \mathbf{U}(\mathbf{z}) \bar{\varphi} d_{\mathbf{z}} S,
\end{aligned}
$$

where

$$
W^{(2)}(\mathbf{V})=\alpha_{1}|\nabla \varphi|^{2}-\xi_{2}|\varphi|^{2}+\tau^{*} \nabla \theta \nabla \bar{\varphi}-m \theta \bar{\varphi}+\nu_{1} \operatorname{div} \mathbf{u} \bar{\varphi}
$$

On account of the second equation of (4.6), the boundary condition (5.5) in the case $K=I I I$ and (5.7) in the case $K=I V$, from (5.11) we get

$$
\int_{\Omega^{+}} W^{(2)}(\mathbf{V}) d \mathbf{x}=0 .
$$

Quite similarly, from third equation of (4.6) and conditions (5.5) and (5.7) it follows that

$$
\int_{\Omega^{+}} W^{(3)}(\mathbf{V}) d \mathbf{x}=0,
$$

where

$$
W^{(3)}(\mathbf{V})=k|\nabla \theta|^{2}-c_{1}|\theta|^{2}+\zeta_{1} \nabla \varphi \nabla \bar{\theta}-m_{1} \varphi \bar{\theta}-\beta_{1} \operatorname{div} \mathbf{u} \bar{\theta}
$$


Hence, from (5.10), (5.13) and (5.14) we have

$$
\int_{\Omega^{+}}\left\{\omega T_{0} \operatorname{Im}\left[W^{(1)}(\mathbf{V})+W^{(2)}(\mathbf{V})\right]-\operatorname{Re} W^{(3)}(\mathbf{V})\right\} d \mathbf{x}=0
$$

On the other hand, by virtue of (2.7), (4.5) and the identity

$$
\operatorname{Im}\left(b_{1} \varphi \operatorname{div} \overline{\mathbf{u}}+\nu_{1} \operatorname{div} \mathbf{u} \bar{\varphi}\right)=-\omega\left(b^{*}+\nu^{*}\right) \operatorname{Re}(\varphi \operatorname{div} \overline{\mathbf{u}})
$$

from $(5.9),(5.12),(5.15)$ we obtain

$$
\begin{aligned}
\operatorname{Im}[ & \left.W^{(1)}(\mathbf{V})+W^{(2)}(\mathbf{V})\right] \\
& =-\omega W\left(\mathbf{u}, \lambda^{*}, \mu^{*}\right)-\omega\left(b^{*}+\nu^{*}\right) \operatorname{Re}(\varphi \operatorname{div} \overline{\mathbf{u}})-\beta \operatorname{Im}(\theta \operatorname{div} \overline{\mathbf{u}}) \\
& -\omega \alpha^{*}|\nabla \varphi|^{2}-\omega \xi^{*}|\varphi|^{2}+\tau^{*} \operatorname{Im}(\nabla \theta \nabla \bar{\varphi})-m \operatorname{Im}(\theta \bar{\varphi}),
\end{aligned}
$$

$\operatorname{Re} W^{(3)}(\mathbf{V})=k|\nabla \theta|^{2}-\omega \beta T_{0} \operatorname{Im}(\theta \operatorname{div} \overline{\mathbf{u}})-\omega \zeta^{*} \operatorname{Im}(\nabla \theta \nabla \bar{\varphi})-\omega m T_{0} \operatorname{Im}(\theta \bar{\varphi})$

and consequently, we get

$$
\begin{aligned}
\omega T_{0} \operatorname{Im} & {\left[W^{(1)}(\mathbf{V})+W^{(2)}(\mathbf{V})\right]-\operatorname{Re} W^{(3)}(\mathbf{V}) } \\
= & -\omega^{2} T_{0}\left[W\left(\mathbf{u}, \lambda^{*}, \mu^{*}\right)+\left(b^{*}+\nu^{*}\right) \operatorname{Re}(\varphi \operatorname{div} \overline{\mathbf{u}})+\xi^{*}|\varphi|^{2}\right] \\
& -\left[k|\nabla \theta|^{2}-\omega\left(\zeta^{*}+\tau^{*} T_{0}\right) \operatorname{Im}(\nabla \theta \nabla \bar{\varphi})+\omega^{2} T_{0} \alpha^{*}|\nabla \varphi|^{2}\right] \\
= & -\omega^{2} T_{0}\left[\frac{1}{3}\left(3 \lambda^{*}+2 \mu^{*}\right)|\operatorname{div} \mathbf{u}|^{2}+\left(b^{*}+\nu^{*}\right) \operatorname{Re}(\varphi \operatorname{div} \overline{\mathbf{u}})+\xi^{*}|\varphi|^{2}\right] \\
& -\omega^{2} T_{0} \mu^{*}\left[\frac{1}{2} \sum_{l, j=1 ; l \neq j}^{3}\left|\frac{\partial u_{j}}{\partial x_{l}}+\frac{\partial u_{l}}{\partial x_{j}}\right|^{2}+\frac{1}{3} \sum_{l, j=1}^{3}\left|\frac{\partial u_{l}}{\partial x_{l}}-\frac{\partial u_{j}}{\partial x_{j}}\right|^{2}\right] \\
& -\left[k|\nabla \theta|^{2}-\omega\left(\zeta^{*}+\tau^{*} T_{0}\right) \operatorname{Im}(\nabla \theta \nabla \bar{\varphi})+\omega^{2} T_{0} \alpha^{*}|\nabla \varphi|^{2}\right] .
\end{aligned}
$$


Obviously, with the help of (4.10) it follows that

$$
\begin{aligned}
& \frac{1}{3}\left(3 \lambda^{*}+2 \mu^{*}\right)|\operatorname{div} \mathbf{u}|^{2}-\left(b^{*}+\nu^{*}\right) \operatorname{Re}(\varphi \operatorname{div} \overline{\mathbf{u}})+\xi^{*}|\varphi|^{2} \geq 0, \\
& k|\nabla \theta|^{2}-\omega\left(\zeta^{*}+\tau^{*} T_{0}\right) \operatorname{Im}(\nabla \theta \nabla \bar{\varphi})+\omega^{2} T_{0} \alpha^{*}|\nabla \varphi|^{2} \geq 0 .
\end{aligned}
$$

From (5.17) we have $\omega T_{0} \operatorname{Im}\left[W^{(1)}(\mathbf{V})+W^{(2)}(\mathbf{V})\right]-\operatorname{Re} W^{(3)}(\mathbf{V}) \leq 0$. On the basis of (5.16) we obtain

$$
\omega T_{0} \operatorname{Im}\left[W^{(1)}(\mathbf{V})+W^{(2)}(\mathbf{V})\right]-\operatorname{Re} W^{(3)}(\mathbf{V})=0
$$

It is easy to verify that the last equation and (5.17) lead to the following relations

$$
\begin{aligned}
& \frac{1}{3}\left(3 \lambda^{*}+2 \mu^{*}\right)|\operatorname{div} \mathbf{u}|^{2}-\left(b^{*}+\nu^{*}\right) \operatorname{Re}(\varphi \operatorname{div} \overline{\mathbf{u}})+\xi^{*}|\varphi|^{2}=0, \\
& \frac{1}{2} \sum_{l, j=1 ; l \neq j}^{3}\left|\frac{\partial u_{j}}{\partial x_{l}}+\frac{\partial u_{l}}{\partial x_{j}}\right|^{2}+\frac{1}{3} \sum_{l, j=1}^{3}\left|\frac{\partial u_{l}}{\partial x_{l}}-\frac{\partial u_{j}}{\partial x_{j}}\right|^{2}=0 \\
& k|\nabla \theta|^{2}-\omega\left(\zeta^{*}+\tau^{*} T_{0}\right) \operatorname{Im}(\nabla \theta \nabla \bar{\varphi})+\omega^{2} T_{0} \alpha^{*}|\nabla \varphi|^{2}=0 .
\end{aligned}
$$

On account of inequalities of (4.10) from (5.18) we have

$$
\begin{aligned}
& \operatorname{div} \mathbf{u}(\mathbf{x})=0, \quad \frac{\partial u_{j}(\mathbf{x})}{\partial x_{l}}+\frac{\partial u_{l}(\mathbf{x})}{\partial x_{j}}=0 \\
& \frac{\partial u_{l}(\mathbf{x})}{\partial x_{l}}-\frac{\partial u_{j}(\mathbf{x})}{\partial x_{j}}=0, \quad \varphi(\mathbf{x})=0 \\
& \theta(\mathbf{x})=\text { const }, \quad l, j=1,2,3
\end{aligned}
$$

for $\mathbf{x} \in \Omega^{+}$. On the basis of (5.19) from third equation of system (4.6) it follows that $\theta(\mathbf{x})=0$. In view of $(4.7)$ we get $W\left(\mathbf{u}, \lambda_{1}, \mu_{1}\right)=0$. From (5.9) and (5.10) we obtain $\mathbf{u}(\mathbf{x})=\mathbf{0}$. Thus, $\mathbf{V}(\mathbf{x})=\mathbf{0}$ for $\mathbf{x} \in \Omega^{+}$.

Lemma 5.1. If $\mathbf{V}=(\mathbf{u}, \varphi, \theta) \in C^{2}(\Omega)$ is a solution of the system (4.9) for 
$\mathbf{x} \in \Omega$, then

$$
\mathbf{u}(\mathbf{x})=\sum_{j=1}^{4} \mathbf{u}^{(j)}(\mathbf{x}), \quad \varphi(\mathbf{x})=\sum_{l=1}^{3} \varphi^{(l)}(\mathbf{x}), \quad \theta(\mathbf{x})=\sum_{l=1}^{3} \theta^{(l)}(\mathbf{x})
$$

where $\Omega$ is an arbitrary domain in $R^{3}, \mathbf{u}^{(j)}, \varphi^{(l)}$ and $\theta^{(l)}$ satisfy the following equations

$$
\begin{aligned}
& \left(\Delta+\kappa_{j}^{2}\right) \mathbf{u}^{(j)}(\mathbf{x})=\mathbf{0}, \quad\left(\Delta+\kappa_{l}^{2}\right) \varphi^{(l)}(\mathbf{x})=0, \\
& \left(\Delta+\kappa_{l}^{2}\right) \theta^{(l)}(\mathbf{x})=0, \quad l=1,2,3, \quad j=1,2,3,4 .
\end{aligned}
$$

Proof. Applying the operator div to the first equation of (4.6) we have

$$
\begin{aligned}
& \left(\mu_{2} \Delta+\rho \omega^{2}\right) \operatorname{div} \mathbf{u}+b_{1} \Delta \varphi-\beta \Delta \theta=\mathbf{0}, \\
& \left(\alpha_{1} \Delta+\xi_{2}\right) \varphi+\left(\tau^{*} \Delta+m\right) \theta-\nu_{1} \operatorname{div} \mathbf{u}=0, \\
& \left(k \Delta+c_{1}\right) \theta+\left(\zeta_{1} \Delta+m_{1}\right) \varphi+\beta_{1} \operatorname{div} \mathbf{u}=0 .
\end{aligned}
$$

Clearly, from system (5.22) it follows that

$$
\Lambda_{1}(\Delta) \operatorname{div} \mathbf{u}=0, \quad \Lambda_{1}(\Delta) \varphi=0, \quad \Lambda_{1}(\Delta) \theta=0 .
$$

Now, applying the operator $\Lambda_{1}(\Delta)$ to the first equation of (4.6) and using (5.23) we obtain

$$
\Lambda_{1}(\Delta)\left(\Delta+\kappa_{4}^{2}\right) \mathbf{u}=\mathbf{0} .
$$

We introduce the notation

$$
\begin{aligned}
& \mathbf{u}^{(j)}=\left[\prod_{p=1 ; p \neq j}^{4}\left(\kappa_{p}^{2}-\kappa_{j}^{2}\right)^{-1}\left(\Delta+\kappa_{p}^{2}\right)\right] \mathbf{u}, \quad j=1,2,3,4, \\
& \varphi^{(l)}=\left[\prod_{p=1 ; p \neq l}^{3}\left(\kappa_{p}^{2}-\kappa_{l}^{2}\right)^{-1}\left(\Delta+\kappa_{p}^{2}\right)\right] \varphi, \quad l=1,2,3 .
\end{aligned}
$$


By virtue of (5.23) and (5.24) the relations (5.20) and (5.21) can be easily obtained from (5.25). $\diamond$

Now let us establish the uniqueness of a regular solution of the external BVPs.

Theorem 5.2. If condition (4.10) is satisfied, then the external BVP $(K)_{\mathbf{F}, \mathbf{f}}^{-}$ admits at most one regular solution, where $K=I I I, I V$.

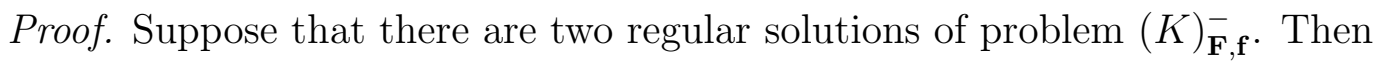
their difference $\mathbf{V}$ corresponds to zero data $(\mathbf{F}=\mathbf{f}=\mathbf{0})$, i.e. $\mathbf{V}$ is a regular solution of the problem $(K)_{\mathbf{0}, \mathbf{0}}^{-}$.

Let $\Omega_{r}$ be a sphere of sufficiently large radius $r$ so that $\bar{\Omega}^{+} \subset \Omega_{r}$. By virtue of (4.6) and the homogeneous boundary condition $(\mathbf{f}=\mathbf{0})$, the formula (5.8) for the domain $\Omega_{r}^{-}=\Omega^{-} \cap \Omega_{r}$ can be rewritten as

$$
\int_{\Omega_{r}^{-}} W^{(1)}(\mathbf{V}) d \mathbf{x}=\int_{S_{r}} \mathbf{P}^{(1)}\left(\mathbf{D}_{\mathbf{z}}, \mathbf{n}\right) \mathbf{V}(\mathbf{z}) \cdot \mathbf{u}(\mathbf{z}) d_{\mathbf{z}} S,
$$

where $S_{r}$ is the boundary of the sphere $\Omega_{r}$ and $\mathbf{n}(\mathbf{z})$ is the external (with respect to $\Omega_{r}$ ) unit normal vector to $S_{r}$ at $\mathbf{z}$. From (5.26) we have

$$
\int_{\Omega^{-}} W^{(1)}(\mathbf{V}) d \mathbf{x}=\lim _{r \rightarrow \infty} \int_{S_{r}} \mathbf{P}^{(1)}\left(\mathbf{D}_{\mathbf{z}}, \mathbf{n}\right) \mathbf{V}(\mathbf{z}) \cdot \mathbf{u}(\mathbf{z}) d_{\mathbf{z}} S .
$$

In addition, on account of (4.65) we have

$$
\lim _{r \rightarrow \infty} \int_{S_{r}} \mathbf{P}^{(1)}\left(\mathbf{D}_{\mathbf{z}}, \mathbf{n}\right) \mathbf{V}(\mathbf{z}) \cdot \mathbf{u}(\mathbf{z}) d_{\mathbf{z}} S=0
$$

Hence, from (5.27) we get

$$
\int_{\Omega^{-}} W^{(1)}(\mathbf{V}) d \mathbf{x}=0
$$


On the other hand, by virtue of (4.65) we obtain

$$
\int_{\Omega^{-}} W^{(j)}(\mathbf{V}) d \mathbf{x}=0, \quad j=2,3 .
$$

Quite similarly, as in theorem 5.1, on the basis of (4.6), (4.10) from (5.28) and (5.29) it follows that $\mathbf{U}(\mathbf{x})=\mathbf{0}$ for $\mathbf{x} \in \Omega^{-}$. $\diamond$

Remark 5.1. In Pamplona et al. [103], the uniqueness and analyticity of solutions of the initial-BVPs in the linear theory of thermoviscoelasticity for anisotropic Kelvin-Voigt materials with voids are proved.

\subsection{Basic properties of thermoelastopotentials}

On the basis of Somigliana-type integral representation of regular vector (see, (4.62) and (4.63)) we introduce the following notations

$$
\begin{aligned}
& \boldsymbol{z}^{(1)}(\mathbf{x}, \mathbf{g})=\int_{S} \boldsymbol{\Theta}(\mathbf{x}-\mathbf{y}) \mathbf{g}(\mathbf{y}) d_{\mathbf{y}} S \\
& \boldsymbol{z}^{(2)}(\mathbf{x}, \mathbf{g})=\int_{S}\left[\tilde{\mathbf{P}}\left(\mathbf{D}_{\mathbf{y}}, \mathbf{n}(\mathbf{y})\right) \boldsymbol{\Theta}^{\top}(\mathbf{x}-\mathbf{y})\right]^{\top} \mathbf{g}(\mathbf{y}) d_{\mathbf{y}} S \\
& \boldsymbol{z}^{(3)}\left(\mathbf{x}, \boldsymbol{\phi}, \Omega^{ \pm}\right)=\int_{\Omega^{ \pm}} \boldsymbol{\Theta}(\mathbf{x}-\mathbf{y}) \boldsymbol{\phi}(\mathbf{y}) d \mathbf{y},
\end{aligned}
$$

where $\mathbf{g}$ and $\boldsymbol{\phi}$ are five-component vectors.

As in classical theory of thermoelasticity (see, e.g. Kupradze et al. [83]), the vector functions $\boldsymbol{Z}^{(1)}(\mathbf{x}, \mathbf{g}), \boldsymbol{z}^{(2)}(\mathbf{x}, \mathbf{g})$ and $\boldsymbol{z}^{(3)}\left(\mathbf{x}, \boldsymbol{\phi}, \Omega^{ \pm}\right)$are called single-layer, double-layer and volume potentials in the linear theory of thermoviscoelasticity for Kelvin-Voigt materials with voids, respectively.

Obviously, on the basis of (4.62) and (4.63), the regular vector $\mathbf{V}(\mathbf{x})$ in $\Omega^{+}$ 
and $\Omega^{-}$is represented by sum of the thermoelastopotentials as follows

$\mathbf{V}(\mathbf{x})=\boldsymbol{Z}^{(2)}\left(\mathbf{x},\{\mathbf{V}\}^{+}\right)-\boldsymbol{z}^{(1)}\left(\mathbf{x},\{\mathbf{R V}\}^{+}\right)+\boldsymbol{Z}^{(3)}\left(\mathbf{x}, \mathcal{A} \mathbf{V}, \Omega^{+}\right) \quad$ for $\quad \mathbf{x} \in \Omega^{+}$

and

$\mathbf{V}(\mathbf{x})=-\boldsymbol{Z}^{(2)}\left(\mathbf{x},\{\mathbf{V}\}^{-}\right)+\boldsymbol{Z}^{(1)}\left(\mathbf{x},\{\mathbf{R V}\}^{-}\right)+\boldsymbol{Z}^{(3)}\left(\mathbf{x}, \mathcal{A V}, \Omega^{-}\right) \quad$ for $\quad \mathbf{x} \in \Omega^{-}$ respectively.

The basic properties of potentials $\boldsymbol{z}^{(1)}(\mathbf{x}, \mathbf{g}), \boldsymbol{z}^{(2)}(\mathbf{x}, \mathbf{g})$ and $\boldsymbol{z}^{(3)}\left(\mathbf{x}, \boldsymbol{\phi}, \Omega^{ \pm}\right)$are given in the following theorems.

Theorem 5.3. If $S \in C^{2, p}, \mathbf{g} \in C^{1, p^{\prime}}(S), 0<p^{\prime}<p \leq 1$, then:

a)

$$
\boldsymbol{z}^{(1)}(\cdot, \mathbf{g}) \in C^{0, p^{\prime}}\left(R^{3}\right) \cap C^{2, p^{\prime}}\left(\bar{\Omega}^{ \pm}\right) \cap C^{\infty}\left(\Omega^{ \pm}\right),
$$

b)

$$
\mathcal{A}\left(\mathbf{D}_{\mathbf{x}}\right) \boldsymbol{Z}^{(1)}(\mathbf{x}, \mathbf{g})=\mathbf{0} \quad \text { for } \quad \mathbf{x} \in \Omega^{ \pm}
$$

c)

$$
\left\{\mathbf{R}\left(\mathbf{D}_{\mathbf{z}}, \mathbf{n}(\mathbf{z})\right) \mathbf{z}^{(1)}(\mathbf{z}, \mathbf{g})\right\}^{ \pm}=\mp \frac{1}{2} \mathbf{g}(\mathbf{z})+\mathbf{R}\left(\mathbf{D}_{\mathbf{z}}, \mathbf{n}(\mathbf{z})\right) \mathbf{z}^{(1)}(\mathbf{z}, \mathbf{g}) \quad \text { for } \quad \mathbf{z} \in S
$$

d) $\mathbf{R}\left(\mathbf{D}_{\mathbf{z}}, \mathbf{n}(\mathbf{z})\right) \boldsymbol{z}^{(1)}(\mathbf{z}, \mathbf{g})$ is a singular integral for $\mathbf{z} \in S$.

Theorem 5.4. If $S \in C^{2, p}, \mathbf{g} \in C^{1, p^{\prime}}(S), 0<p^{\prime}<p \leq 1$, then:

a)

$$
\boldsymbol{z}^{(2)}(\cdot, \mathbf{g}) \in C^{1, p^{\prime}}\left(\bar{\Omega}^{ \pm}\right) \cap C^{\infty}\left(\Omega^{ \pm}\right)
$$

b)

$$
\mathcal{A}\left(\mathbf{D}_{\mathbf{x}}\right) \boldsymbol{Z}^{(2)}(\mathbf{x}, \mathbf{g})=\mathbf{0} \quad \text { for } \quad \mathbf{x} \in \Omega^{ \pm}
$$

c)

$$
\left\{\boldsymbol{z}^{(2)}(\mathbf{z}, \mathbf{g})\right\}^{ \pm}= \pm \frac{1}{2} \mathbf{g}(\mathbf{z})+\boldsymbol{z}^{(2)}(\mathbf{z}, \mathbf{g}) \quad \text { for } \quad \mathbf{z} \in S
$$

d) $\mathbf{z}^{(2)}(\mathbf{z}, \mathbf{g})$ is a singular integral for $\mathbf{z} \in S$, 
e)

$$
\left\{\mathbf{R}\left(\mathbf{D}_{\mathbf{z}}, \mathbf{n}(\mathbf{z})\right) \boldsymbol{Z}^{(2)}(\mathbf{z}, \mathbf{g})\right\}^{+}=\left\{\mathbf{R}\left(\mathbf{D}_{\mathbf{z}}, \mathbf{n}(\mathbf{z})\right) \boldsymbol{Z}^{(2)}(\mathbf{z}, \mathbf{g})\right\}^{-} \quad \text { for } \quad \mathbf{z} \in S
$$

Theorem 5.5. If $S \in C^{1, p}, \phi \in C^{0, p^{\prime}}\left(\Omega^{+}\right), 0<p^{\prime}<p \leq 1$, then:

a)

$$
\mathcal{A}^{(3)}\left(\cdot, \phi, \Omega^{+}\right) \in C^{1, p^{\prime}}\left(R^{3}\right) \cap C^{2}\left(\Omega^{+}\right) \cap C^{2, p^{\prime}}\left(\bar{\Omega}_{0}^{+}\right)
$$

b)

$$
\mathcal{A}\left(\mathbf{D}_{\mathbf{x}}\right) \boldsymbol{Z}^{(3)}\left(\mathbf{x}, \phi, \Omega^{+}\right)=\phi(\mathbf{x}) \quad \text { for } \quad \mathrm{x} \in \Omega^{+}
$$

where $\Omega_{0}^{+}$is a domain in $\mathbb{R}^{3}$ and $\Omega_{0}^{+} \subset \Omega^{+}$.

Theorem 5.6. If $S \in C^{1, p}, \operatorname{supp} \phi=\Omega \subset \Omega^{-}, \quad \phi \in C^{0, p^{\prime}}\left(\Omega^{-}\right), 0<p^{\prime}<p \leq 1$, then:

a)

$$
\boldsymbol{z}^{(3)}\left(\cdot, \phi, \Omega^{-}\right) \in C^{1, p^{\prime}}\left(R^{3}\right) \cap C^{2}\left(\Omega^{-}\right) \cap C^{2, p^{\prime}}\left(\bar{\Omega}_{0}^{-}\right)
$$

b)

$$
\mathcal{A}\left(\mathbf{D}_{\mathbf{x}}\right) \boldsymbol{Z}^{(3)}\left(\mathbf{x}, \phi, \Omega^{-}\right)=\phi(\mathbf{x}) \quad \text { for } \quad \mathrm{x} \in \Omega^{-},
$$

where $\Omega$ is a finite domain in $\mathbb{R}^{3}$ and $\bar{\Omega}_{0}^{-} \subset \Omega^{-}$.

Theorems 5.3 to 5.6 can be proved similarly to the corresponding theorems in the classical theory of thermoelasticity (for details, see Kupradze et al. [83], Ch. $\mathrm{X})$. 
We introduce the notation

$$
\begin{aligned}
& \mathcal{K}^{(5)} \mathbf{g}(\mathbf{z}) \equiv \frac{1}{2} \mathbf{g}(\mathbf{z})+\mathbf{Z}^{(2)}(\mathbf{z}, \mathbf{g}), \\
& \mathcal{K}^{(6)} \mathbf{g}(\mathbf{z}) \equiv-\frac{1}{2} \mathbf{g}(\mathbf{z})+\mathbf{P}\left(\mathbf{D}_{\mathbf{z}}, \mathbf{n}(\mathbf{z})\right) \boldsymbol{Z}^{(1)}(\mathbf{z}, \mathbf{g}), \\
& \mathcal{K}^{(7)} \mathbf{g}(\mathbf{z}) \equiv-\frac{1}{2} \mathbf{g}(\mathbf{z})+\mathbf{z}^{(2)}(\mathbf{z}, \mathbf{g}), \\
& \mathcal{K}^{(8)} \mathbf{g}(\mathbf{z}) \equiv \frac{1}{2} \mathbf{g}(\mathbf{z})+\mathbf{P}\left(\mathbf{D}_{\mathbf{z}}, \mathbf{n}(\mathbf{z})\right) \boldsymbol{Z}^{(1)}(\mathbf{z}, \mathbf{g}), \\
& \tilde{\mathcal{K}}_{\chi} \mathbf{g}(\mathbf{z}) \equiv-\frac{1}{2} \mathbf{g}(\mathbf{z})+\chi \boldsymbol{z}^{(2)}(\mathbf{z}, \mathbf{g}) \quad \text { for } \quad \mathbf{z} \in S,
\end{aligned}
$$

where $\chi$ is a complex parameter. On the basis of theorems 5.3 and $5.4, \mathcal{K}^{(j)}(j=$ $5,6,7,8)$ and $\tilde{\mathcal{K}}_{\chi}$ are singular integral operators (see Mikhlin [81], Kupradze et al. $[83])$.

In the sequel we need the following Lemmas.

Lemma 5.2. If condition (4.10) is satisfied, then the singular integral operators $\mathcal{K}^{(j)}(j=5,6,7,8)$ are of the normal type.

Proof: Let $\boldsymbol{\sigma}^{(j)}=\left(\sigma_{l m}^{(j)}\right)_{5 \times 5}$ be the symbol of the singular integral operator $\mathcal{K}^{(j)}(j=5,6,7,8)$ (see, Mikhlin [81]). Taking into account (5.32) we find

$$
\operatorname{det} \boldsymbol{\sigma}^{(j)}=\left(-\frac{1}{2}\right)^{5}\left[1-\frac{\mu_{1}^{2}}{\left(\lambda_{1}+2 \mu_{1}\right)^{2}}\right]=-\frac{\left(\lambda_{1}+\mu_{1}\right)\left(\lambda_{1}+3 \mu_{1}\right)}{32\left(\lambda_{1}+2 \mu_{1}\right)^{2}} .
$$

Keeping in mind the relations (4.10) from (5.33) we have

$$
\operatorname{det} \boldsymbol{\sigma}^{(j)} \neq 0
$$

Hence, the operator $\mathcal{K}^{(j)}$ is of the normal type, where $j=5,6,7,8$. $\diamond$

Lemma 5.3. If $\mathcal{L}$ is a continuous curve on the complex plane connecting the origin with the point $\chi_{0}$ and $\tilde{\mathcal{K}}_{\chi}$ is a normal type operator for any $\chi \in \mathcal{L}$, then 
the index of the operator $\tilde{\mathcal{K}}_{\chi_{0}}$ vanishes, i.e.

$$
\text { ind } \tilde{\mathcal{K}}_{\chi_{0}}=0
$$

Lemma 5.3 for an arbitrary singular integral operator is proved in Kupradze et al. [83], Ch. IV.

Lemma 5.4. If conditions (4.10) are satisfied, then the Fredholm's theorems are valid for the singular integral operator $\tilde{\mathcal{K}}^{(j)}\left(\tilde{\mathcal{K}}^{(j)}\right.$ is Fredholmian), where $j=5,6,7,8$.

Proof: Let $\tilde{\boldsymbol{\sigma}}_{\chi}$ and ind $\tilde{\mathcal{K}}_{\chi}$ be the symbol and the index of the operator $\tilde{\mathcal{K}}_{\chi}$, respectively. It may be easily shown that

$$
\operatorname{det} \tilde{\boldsymbol{\sigma}}_{\chi}=-\frac{\left(\lambda_{1}+2 \mu_{1}\right)^{2}-\mu_{1}^{2} \chi^{2}}{32\left(\lambda_{1}+2 \mu_{1}\right)^{2}}
$$

and $\operatorname{det} \tilde{\boldsymbol{\sigma}}_{\chi}$ vanishes only at two points $\chi_{1}$ and $\chi_{2}$ of the complex plane. By virtue of (5.34) and $\operatorname{det} \tilde{\boldsymbol{\sigma}}_{1}=\operatorname{det} \boldsymbol{\sigma}^{(5)}$ we get $\chi_{l} \neq 1$ for $l=1,2$. By Lemma 5.3 we have

$$
\text { ind } \mathcal{K}^{(5)}=\text { ind } \tilde{\mathcal{K}}_{1}=0
$$

Quite similarly we obtain ind $\mathcal{K}^{(6)}=0$. Obviously, the operators $\mathcal{K}^{(7)}$ and $\mathcal{K}^{(8)}$ are the adjoint operators for $\mathcal{K}^{(6)}$ and $\mathcal{K}^{(5)}$, respectively. Evidently,

$$
\text { ind } \mathcal{K}^{(7)}=- \text { ind } \mathcal{K}^{(6)}=0, \quad \text { ind } \mathcal{K}^{(8)}=- \text { ind } \mathcal{K}^{(5)}=0 \text {. }
$$

Thus, the singular integral operator $\mathcal{K}^{(j)}(j=5,6,7,8)$ is of the normal type with an index equal to zero. Consequently, the Fredholm's theorems are valid for $\mathcal{K}^{(j)}$.

\subsection{Existence theorems}

Obviously, by theorems 5.5 and 5.6 the volume potential $\boldsymbol{Z}^{(3)}\left(\mathbf{x}, \mathbf{F}, \Omega^{ \pm}\right)$is a regular solution of the system of nonhomogeneous equations (4.8), where $\mathbf{F} \in$ 
$C^{0, p^{\prime}}\left(\Omega^{ \pm}\right), 0<p^{\prime} \leq 1 ; \operatorname{supp} \mathbf{F}$ is a finite domain in $\Omega^{-}$. Therefore, further we will consider problem $(K)_{\mathbf{0}, \mathbf{f}}^{ \pm}$for $K=I I I, I V$.

Now we prove the existence theorems of the regular (classical) solutions of problems $(K)_{\mathbf{0}, \mathbf{f}}^{+}$and $(K)_{\mathbf{0}, \mathbf{f}}^{-}$for $K=I I I, I V$.

Problem $(I I I)_{\mathbf{0}, \mathbf{f}}^{+}$. We seek a regular solution to internal BVP $(I I I)_{\mathbf{0}, \mathbf{f}}^{+}$in the form

$$
\mathbf{V}(\mathbf{x})=\mathbf{Z}^{(2)}(\mathbf{x}, \mathbf{g}) \quad \text { for } \quad \mathbf{x} \in \Omega^{+},
$$

where $\mathbf{g}$ is the required five-component vector function.

By theorem 5.4 the vector function $\mathbf{V}$ is a solution of homogeneous equation (4.9) for $\mathbf{x} \in \Omega^{+}$. Keeping in mind the boundary condition (5.1), using (5.31) and (5.32), from (5.35) we obtain, for determining the unknown vector $\mathbf{g}$, a singular integral equation

$$
\mathcal{K}^{(5)} \mathbf{g}(\mathbf{z})=\mathbf{f}(\mathbf{z}) \quad \text { for } \quad \mathbf{z} \in S .
$$

By lemma 5.4 the Fredholm's theorems are valid for operator $\mathcal{K}^{(5)}$. We prove that (5.36) is always solvable for an arbitrary vector $\mathbf{f}$. Let us consider the associate homogeneous equation

$$
\mathcal{K}^{(8)} \mathbf{h}_{0}(\mathbf{z})=\mathbf{0} \quad \text { for } \quad \mathbf{z} \in S \text {, }
$$

where $\mathbf{h}_{0}$ is the required five-component vector function. Now we prove that (5.37) has only the trivial solution.

Indeed, let $\mathbf{h}_{0}$ be a solution of the homogeneous equation (5.37). On the basis of theorem 5.3 and (5.37) the vector function $\tilde{\mathbf{V}}(\mathbf{x})=\mathbf{z}^{(1)}\left(\mathbf{x}, \mathbf{h}_{0}\right)$ is a regular solution of the homogeneous BVP $(I V)_{\mathbf{0}, \mathbf{0}}^{-}$. Using theorem 5.2, the problem $(I V)_{\mathbf{0}, \mathbf{0}}^{-}$ has only the trivial solution, that is

$$
\tilde{\mathbf{V}}(\mathbf{x})=\mathbf{0} \quad \text { for } \quad \mathbf{x} \in \Omega^{-}
$$

On the other hand, by theorem 5.3 and (5.38) we get

$$
\{\tilde{\mathbf{V}}(\mathbf{z})\}^{+}=\{\tilde{\mathbf{V}}(\mathbf{z})\}^{-}=\mathbf{0} \quad \text { for } \quad \mathbf{z} \in S
$$


i.e., the vector $\tilde{\mathbf{V}}(\mathbf{x})$ is a regular solution of problem $(I I I)_{\mathbf{0}, \mathbf{0}}^{+}$. Using theorem 5.1, the problem $(I I I)_{\mathbf{0}, \mathbf{0}}^{+}$has only the trivial solution, that is

$$
\tilde{\mathbf{V}}(\mathbf{x})=\mathbf{0} \quad \text { for } \quad \mathbf{x} \in \Omega^{+}
$$

By virtue of (5.38), (5.39) and identity (5.30) we obtain

$$
\mathbf{h}_{0}(\mathbf{z})=\left\{\mathbf{R}\left(\mathbf{D}_{\mathbf{z}}, \mathbf{n}\right) \tilde{\mathbf{V}}(\mathbf{z})\right\}^{-}-\left\{\mathbf{R}\left(\mathbf{D}_{\mathbf{z}}, \mathbf{n}\right) \tilde{\mathbf{V}}(\mathbf{z})\right\}^{+}=\mathbf{0} \quad \text { for } \quad \mathbf{z} \in S
$$

Thus, the homogeneous equation (5.37) has only the trivial solution and therefore (5.36) is always solvable for an arbitrary vector $\mathbf{f}$.

We have thereby proved

Theorem 5.7. If $S \in C^{2, p}, \mathbf{f} \in C^{1, p^{\prime}}(S), 0<p^{\prime}<p \leq 1$, then a regular solution of problem $(I I I)_{\mathbf{0}, \mathbf{f}}^{+}$exists, is unique and is represented by double-layer potential (5.35), where $\mathbf{g}$ is a solution of the singular integral equation (5.36) which is always solvable for an arbitrary vector $\mathbf{f}$.

Problem $(I V)_{\mathbf{0}, \mathbf{f}}^{-}$. We seek a regular solution to problem $(I V)_{\mathbf{0}, \mathbf{f}}^{-}$in the form

$$
\mathbf{V}(\mathbf{x})=\mathbf{Z}^{(1)}(\mathbf{x}, \mathbf{h}) \quad \text { for } \quad \mathbf{x} \in \Omega^{-},
$$

where $\mathbf{h}$ is the required five-component vector function.

Obviously, by theorem 5.3 the vector function $\mathbf{V}$ is a solution of (4.9) for $\mathbf{x} \in \Omega^{-}$. Keeping in mind the boundary condition (5.4), using (5.30) and (5.32), from (5.40) we obtain, for determining the unknown vector $\mathbf{h}$, a singular integral equation

$$
\mathcal{K}^{(8)} \mathbf{h}(\mathbf{z})=\mathbf{f}(\mathbf{z}) \quad \text { for } \quad \mathbf{z} \in S .
$$

It has been proved above that the corresponding homogeneous equation (5.37) has only the trivial solution. Hence, it follows that (5.41) is always solvable for an arbitrary vector $\mathbf{f}$.

We have thereby proved

Theorem 5.8. If $S \in C^{2, p}, \mathbf{f} \in C^{0, p^{\prime}}(S), 0<p^{\prime}<p \leq 1$, then a regular solution 
of problem $(I V)_{\mathbf{0}, \mathbf{f}}^{-}$exists, is unique and is represented by single-layer potential (5.40), where $\mathbf{h}$ is a solution of the singular integral equation (5.41) which is always solvable for an arbitrary vector $\mathbf{f}$.

Problem $(I V)_{\mathbf{0}, \mathbf{f}}^{+}$. We seek a regular solution to problem $(I V)_{\mathbf{0}, \mathbf{f}}^{+}$in the form

$$
\mathbf{V}(\mathbf{x})=\boldsymbol{z}^{(1)}(\mathbf{x}, \mathbf{g}) \quad \text { for } \quad \mathbf{x} \in \Omega^{+}
$$

where $\mathbf{g}$ is the required five-component vector function.

Obviously, by theorem 5.3 the vector function $\mathbf{V}$ is a solution of (4.9) for $\mathbf{x} \in \Omega^{+}$. Keeping in mind the boundary condition (5.2), using (5.30) and (5.32), from (5.42) we obtain, for determining the unknown vector $\mathbf{g}$, a singular integral equation

$$
\mathcal{K}^{(6)} \mathbf{g}(\mathbf{z})=\mathbf{f}(\mathbf{z}) \quad \text { for } \quad \mathbf{z} \in S
$$

By lemma 5.4 the Fredholm's theorems are valid for operator $\mathcal{K}^{(6)}$. We prove that (5.43) is always solvable for an arbitrary vector $\mathbf{f}$. Let us consider the corresponding homogeneous equation

$$
\mathcal{K}^{(6)} \mathbf{g}_{0}(\mathbf{z})=\mathbf{0} \quad \text { for } \quad \mathbf{z} \in S
$$

where $\mathbf{g}_{0}$ is the required five-component vector function. Now we prove that (5.44) has only the trivial solution.

Indeed, let $\mathbf{g}_{0}$ be a solution of the homogeneous equation (5.44). On the basis of theorem 5.3 and (5.44) the vector $\tilde{\mathbf{V}}(\mathbf{x})=\mathbf{z}^{(1)}\left(\mathbf{x}, \mathbf{g}_{0}\right)$ is a regular solution of problem $(I V)_{\mathbf{0}, \mathbf{0}}^{+}$. Using theorem 5.1 , the problem $(I V)_{\mathbf{0}, \mathbf{0}}^{+}$has only the trivial solution, i.e., we have the condition (5.39).

On the other hand, by theorem 5.3 and (5.39) we get $\{\tilde{\mathbf{V}}(\mathbf{z})\}^{-}=\mathbf{0}$ for $\mathbf{z} \in S$. Hence, the vector $\tilde{\mathbf{V}}(\mathbf{x})$ is a regular solution of problem $(I I I)_{\mathbf{0}, \mathbf{0}}^{-}$. On the basis of theorem 5.2, the problem $(I I I)_{\mathbf{0}, \mathbf{0}}^{-}$has only the trivial solution, i.e., we obtain the condition (5.38). By virtue of (5.38), (5.39) and identity (5.30) it follows that

$$
\mathbf{g}_{0}(\mathbf{z})=\left\{\mathbf{R}\left(\mathbf{D}_{\mathbf{z}}, \mathbf{n}\right) \tilde{\mathbf{V}}(\mathbf{z})\right\}^{-}-\left\{\mathbf{R}\left(\mathbf{D}_{\mathbf{z}}, \mathbf{n}\right) \tilde{\mathbf{V}}(\mathbf{z})\right\}^{+}=\mathbf{0} \quad \text { for } \quad \mathbf{z} \in S
$$


Thus, the homogeneous equation (5.44) has only a trivial solution and therefore (5.43) is always solvable for an arbitrary vector $\mathbf{f}$.

We have thereby proved

Theorem 5.9. If $S \in C^{2, p}, \mathbf{f} \in C^{0, p^{\prime}}(S), 0<p^{\prime}<p \leq 1$, then a regular solution of problem $(I V)_{\mathbf{0}, \mathbf{f}}^{+}$exists, is unique and is represented by single-layer potential (5.42), where $\mathbf{g}$ is a solution of the singular integral equation (5.43) which is always solvable for an arbitrary vector $\mathbf{f}$.

Problem $(I I I)_{\mathbf{0}, \mathbf{f}}^{-}$. We seek a regular solution to problem $(I I I)_{\mathbf{0}, \mathbf{f}}^{-}$in the form

$$
\mathbf{V}(\mathbf{x})=\boldsymbol{z}^{(2)}(\mathbf{x}, \mathbf{h}) \quad \text { for } \quad \mathbf{x} \in \Omega^{-},
$$

where $\mathbf{h}$ is the required five-component vector function.

Obviously, by theorem 5.3 the vector function $\mathbf{V}$ is a solution of (4.9) for $\mathbf{x} \in \Omega^{-}$. Keeping in mind the boundary condition (5.3), using (5.31) and (5.32), from (5.45) we obtain, for determining the unknown vector $\mathbf{h}$, a singular integral equation

$$
\mathcal{K}^{(7)} \mathbf{h}(\mathbf{z})=\mathbf{f}(\mathbf{z}) \quad \text { for } \quad \mathbf{z} \in S .
$$

It has been proved above that the corresponding associate homogeneous equation (5.44) has only the trivial solution. Hence, it follows that (5.46) is always solvable for an arbitrary vector $\mathbf{f}$.

We have thereby proved

Theorem 5.10. If $S \in C^{2, p}$, $\mathbf{f} \in C^{1, p^{\prime}}(S), 0<p^{\prime}<p \leq 1$, then a regular solution of problem $(I I I)_{\mathbf{0}, \mathbf{f}}^{-}$exists, is unique and is represented by double-layer potential (5.45), where $\mathbf{h}$ is a solution of the singular integral equation (5.46) which is always solvable for an arbitrary vector $\mathbf{f}$.

Remark 5.2. In Pompei and Scalia [44], the BVPs of steady vibrations in a linear theory of homogeneous and isotropic thermoelastic solids with voids is considered. First, integral relations of Betti type are established. The singular solutions corresponding to concentrated sources are used to derive representations of Somigliana type. Then, radiation conditions are introduced and a uniqueness 
result for the external problem is established. The potentials of single layer and double layer are used to reduce the BVPs to singular integral equations for which Fredholm's theorems hold. The existence theorems for external problems are proved.

Remark 5.3. In Pamplona et al. [104], the analyticity of solutions of the initialBVPs in the linear theory of thermoviscoelasticity with microtemperatures for porous materials is proved.

Remark 5.4. Recently, Ieşan and Quintanilla [105] presented a strain gradient theory of thermoviscoelasticity in which the time derivatives of the strain tensors are included in the set of independent constitutive variables. The basic equations of the linear theory are derived. The uniqueness and existence theorems in the considered theory are proved. 


\section{Chapter 6}

\section{Concluding remarks}

In this chapter the basis results of the presented thesis are summarized and some fields of application of there results are analyzed.

In the Chapters 2 to 5 the 3D linear theories of viscoelasticity and thermoviscoelasticity for Kelvin-Voigt materials with voids are investigated and some basic results of the classical theories of elasticity and thermoelasticity are generalized. Indeed:

1. The basic properties of plane harmonic waves in the linear theory of viscoelasticity for Kelvin-Voigt materials with voids are established. There are two $\left(P_{1}\right.$ and $\left.P_{2}\right)$ longitudinal and two $(S H$ and $S V)$ transverse attenuated plane waves in the Kelvin-Voigt material with voids;

2. The explicit expressions of fundamental solutions for the systems of equations of steady vibrations in the linear theories of viscoelasticity and thermoviscoelasticity for Kelvin-Voigt materials with voids is constructed by means of elementary functions. The Green's formulas in the considered theories are obtained;

3. The representations of Galerkin type solutions of the systems of nonhomogeneous equations of steady vibrations in the linear theories of viscoelasticity and thermoviscoelasticity for Kelvin-Voigt materials with voids are presented and the completeness of these solutions is established;

4. The formulas of integral representations of Somigliana type of regular vector and regular (classical) solution are obtained; 


\section{Concluding remarks}

5. The representations of general solutions of the systems of homogeneous equations of steady vibrations in the linear theories of viscoelasticity and thermoviscoelasticity for Kelvin-Voigt materials with voids are obtained by using metaharmonic functions and the completeness of these solutions is established;

6. The basic properties of surface (singe-layer and double-layer) and volume potentials and singular integral operators are presented. These potentials are used to reduce the BVPs to singular integral equations for which Fredholm's theorems hold;

7. The uniqueness and existence theorems for classical solutions of the BVPs of steady vibrations in the linear theories of viscoelasticity and thermoviscoelasticity for Kelvin-Voigt materials with voids are proved by using the potential method and the theory of singular integral equations.

On the basis of results of this thesis it is possible:

1. To investigate the non-classical problems of the $2 \mathrm{D}$ linear theories of viscoelasticity and thermoviscoelasticity for Kelvin-Voigt materials with voids;

2. To investigate the non-classical problems in the modern linear theories of viscoelasticity and thermoviscoelasticity by using potential method and the theory of singular integral equations for the following materials:

a) a viscoelastic composite as a mixture of a porous elastic solid and a Kelvin-

Voigt material (Ieşan [57], Quintanilla [58]),

b) composites modelled as interacting Cosserat continua (Ieşan [59]),

c) mixtures where the individual components is modelled as Kelvin-Voigt viscoelastic materials (Ieşan and Nappa [60]), and

d) Kelvin-Voigt microstretch composite materials (Passarella et al. [66]);

3. To obtain numerical solutions of the BVPs of the linear theory of viscoelasticity for Kelvin-Voigt materials with voids by using of the boundary element method;

4) To establish basic properties of plane harmonic waves in the linear theories of viscoelasticity and thermoviscoelasticity for materials with microstructure (see 


\section{Concluding remarks}

$[58,59,60,66])$

5) To construct the fundamental solutions and to obtain the representations of general solutions for the systems of governing equations of steady vibrations in the linear theories of viscoelasticity and thermoviscoelasticity for materials with microstructure (see $[58,59,60,66])$;

6) To prove the uniqueness and existence theorems in the modern linear theories of viscoelasticity and thermoviscoelasticity for solids with micro and nanostructures. 


\section{References}

1. Svanadze, M. M.: Steady vibrations problem in the theory of viscoelasticity for Kelvin-Voigt materials with voids, Proc. Appl. Math. Mech. 12, 283284 (2012).

2. Svanadze, M. M.: Potential method in the linear theory of viscoelastic materials with voids, J. Elasticity 114, 101-126 (2014).

3. Svanadze, M. M.: On the solutions of equations of the linear thermoviscoelasticity theory for Kelvin-Voigt materials with voids, J. Thermal Stresses, 37, 253-269 (2014).

4. Svanadze, M. M.: Potential method in the theory of thermoviscoelasticity for materials with voids, J. Thermal Stresses, 37, 905-927 (2014).

5. Eringen, A. C.: Mechanics of Continua. R. E. Krieger Publ. Com. Inc, Huntington, New York (1980), 592 p.

6. Truesdell, C., Noll, W.: The Non-linear Field Theories of Mechanics, 3rd ed., Springer-Verlag, Berlin, Haidelberg, New York (2004), 602 p.

7. Christensen, R. M.: Theory of Viscoelasticity, 2nd ed., Dover Publ. Inc., Mineola, New York (2010).

8. Amendola, G., Fabrizio, M., Golden, J. M.: Thermodynamics of Materials with Memory: Theory and Applications. Springer, New York, Dordrecht, Heidelberg, London (2012), 572 p.

9. Shaw, M. T., MacKnight, W. J.: Introduction to Polymer Viscoelasticity, 3rd Ed., John Wiley \& Sons, Inc., Hoboken, New Jersey (2005), 316 p.

10. Ferry, J. D.: Viscoelastic Properties of Polymers, 3rd Ed., John Wiley \& Sons, New York, (1980), 672 p.

11. Lakes, R.: Viscoelastic Materials. Cambridge University Press, Cambridge, 
New York, Melbourne (2009), 461 p.

12. Voyiadjis, G. Z., Song, C. R.: The Coupled Theory of Mixtures in Geomechanics with Applications. Springer-Verlag, Berlin, Heidelberg (2006), 438 p.

13. Polarz, S., Smarsly, B.: Nanoporous Materials. Nanosci. Nanothech. 2, 581612 (2001).

14. Chen, D. L., Yang, P. F., Lai, Y. S.: A review of three-dimensional viscoelastic models with an application to viscoelasticity characterization using nanoindentation. Microelectr. Reliability 52, 541-558 (2012).

15. Gutierrez-Lemini, D.: Engineering Viscoelasticity. Springer, New York, Heidelberg, Dordrecht, London (2014), 353 p.

16. Fabrizio, M., Morro, A.: Mathematical Problems in Linear Viscoelasticity. SIAM, Philadelphia (1992), 203 p.

17. Di Paola, M., Zingales, M.: Exact mechanical models for fractional viscoelastic material, J. Rheology 56, 983 (2012); http://dx.doi.org/10.1122/1.4717492 (22 pages).

18. Di Paola, M., Pinnola, F. P., Zingales, M.: A discrete mechanical model of fractional hereditary materials, Meccanica 48, 1573-1586 (2013).

19. Fabrizio, M., Lazzari, B.: On the existence and the asymptotic stability of solutions for linearly viscoelastic solids, Arch. Ration. Mech. Anal. 116, 139-152 (1991).

20. Appleby, J. A. D., Fabrizio, M., Lazzari, B., Reynolds, D.W.: On exponential asymptotic stability in linear viscoelasticity. Math. Mod. Meth. Appl. Sci. 16, 1677-1694 (2006).

21. Deseri, L., Fabrizio, M., Golden M.: The concept of a minimal state in viscoelasticity: new free energies and applications to PDEs, Arch. Ration. Mech. Anal. 181, 43-96 (2006).

22. Fabrizio, M., Giorgi, C., Morro, A.: Free energies and dissipation properties for systems with memory, Arch. Ration. Mech. Anal. 125, 341-373 (1994).

23. Del Piero, G., Deseri, L.: Monotonic, completely monotonic and exponential relaxation functions in linear viscoelasticity. Quart. Appl. Math. 53, 273300 (1995).

24. Del Piero, G., Deseri, L.: On the analytic expression of the free energy in 
linear viscoelasticity. J. Elasticity 43, 247-278 (1996).

25. Deseri, L., Gentili, G., Golden, M.J.: An explicit formula for the minimum free energy in linear viscoelasticity. J. Elasticity 54, 141-185 (1999).

26. Deseri, L., Golden, J. M., The minimum free energy for continuous spectrum materials, SIAM J. Appl. Math. 67, 869-892 (2007).

27. Graffi, D., Fabrizio, M.: Sulla nozione di stato per materiali viscoelastici di tipo "rate", Atti Acc. Lincei, Rend. Fis. 83, 201-208 (1989).

28. Del Piero G., Deseri L.: On the concepts of state and free energy in linear viscoelasticity, Arch. Ration. Mech. Anal. 138, 1-35 (1997).

29. Nunziato, J. W., Cowin, S. C.: A non-linear theory of elastic materials with voids, Arch. Ration. Mech. Anal. 72, 175-201 (1979)

30. Cowin, S. C., Nunziato, J. W.: Linear elastic materials with voids, J. Elasticity 13, 125-147 (1983)

31. Cowin, S. C., Puri, P.: The classical pressure vessel problems for linear elastic materials with voids, J. Elasticity 13, 157-163 (1983).

32. Puri, P., Cowin, S. C.: Plane waves in linear elastic materials with voids, J. Elast. 15, 167-178 (1985).

33. Ieşan, D.: Some theorems in the theory of elastic materials with voids, J. Elasticity 15, 215-224 (1985).

34. Chandrasekharaiah, D. S.: A uniqueness theorem in the theory of elastic materials with voids, J. Elasticity 18, 173-179 (1987).

35. Chandrasekharaiah, D. S., Cowin, S. C.: Unified complete solutions for the theories of thermoelasticity and poroelasticity. J. Elasticity 21, 121-126 (1989).

36. Scarpetta, E.: On the fundamental solutions in micropolar elasticity with voids, Acta Mechanica 82, 151-158 (1990).

37. Muñoz-Rivera, J. E., Quintanilla, R.: On the time polynomial decay in elastic solids with voids, J. Math. Anal. Appl. 338, 1296-1309 (2008).

38. Pamplona, P., Muñoz-Rivera, J. E., Quintanilla, R.: Stabilization in elastic solids with voids, J. Math. Anal. Appl. 350, 37-49 (2009).

39. Chiriță, S., Ghiba, I. D.: Inhomogeneous plane waves in elastic materials with voids, Wave Motion 47, 333-342 (2010).

40. Chiriţă, S., Ghiba, I. D.: Strong ellipticity and progressive waves in elastic 
materials with voids, Proc. R. Soc. A 466, 439-458 (2010).

41. Ieşan, D.: A theory of thermoelastic materials with voids, Acta Mech. 60, 67-89 (1986).

42. Scalia, A.: A grade consistent micropolar theory of thermoelastic materials with voids, ZAMM 72, 133-140 (1990).

43. Ciarletta, M.: A solution of Galerkin type in the theory of thermoelastic materials with voids, J. Thermal Stresses 14, 409-417 (1991).

44. Pompei, A., Scalia, A.: On the steady vibrations of the thermoelastic porous materials, Int. J. Solids Struc. 31, 2819-2834 (1994).

45. Chiriţă, S., Scalia, A.: On the spatial and temporal behavior in linear thermoelasticity of materials with voids, J. Thermal Stresses 24, 433-455 (2001).

46. Ciarletta, M., Straughan, B.: Thermo-poroacoustic acceleration waves in elastic materials with voids, J. Math. Anal. Appl. 333, 142-150 (2007).

47. Singh, B.: Wave propagation in a generalized thermoelastic material with voids, Appl. Math. Comput. 189, 698-709 (2007).

48. Singh, J., Tomar, S. K.: Plane waves in thermo-elastic material with voids, Mech. Materials 39, 932-940 (2007).

49. Passarella, F.: Some results in micropolar thermoelasticity, Mech. Res. Com. 23, 349-357 (1996).

50. Lebon, G.: A generalized theory of thermoelasticity, J. Tech. Phys. 23, 37-46 (1982).

51. De Cicco, S., Diaco, M.: A theory of thermoelastic materials with voids without energy dissipation, J. Thermal Stresses 25, 493-503 (2002).

52. Cowin, S. C.: The viscoelastic behavior of linear elastic materials with voids, J. Elasticity 15, 185-191 (1985).

53. Ciarletta, M., Scalia, A.: On some theorems in the linear theory of viscoelastic materials with voids, J. Elasticity 25, 149-158 (1991).

54. Scalia, A.: Shock waves in viscoelastic materials with voids. Wave Motion 9, 125-133 (1994).

55. De Cicco, S., Nappa L.: Singular surfaces in thermoviscoelastic materials with voids, J. Elasticity 73, 191-210 (2003).

56. Martínez, F., Quintanilla, R.: Existence, uniqueness and asymptotic behaviour of solutions to the equations of viscoelasticity with voids, Int. J. 
Solids Struct. 35, 3347-3361 (1998).

57. Ieşan, D.: On the theory of viscoelastic mixtures, J. Thermal Stresses 27, 1125-1148 (2004).

58. Quintanilla, R.: Existence and exponential decay in the linear theory of viscoelastic mixtures, European J. Mech. A/Solids 24, 311-324 (2005).

59. Ieşan, D.: A theory of thermoviscoelastic composites modelled as interacting Cosserat continua, J. Thermal Stresses 30, 1269-1289 (2007).

60. Ieşan, D., Nappa, L.: On the theory of viscoelastic mixtures and stability, Math. Mech. Solids 13, 55-80 (2008).

61. Chiriţă, S., Galeş, C., Ghiba, I.D.: On spatial behavior of the harmonic vibrations in Kelvin-Voigt materials, J. Elasticity 93, 81-92 (2008).

62. Ieşan, D.: On a theory of thermoviscoelastic materials with voids, J. Elasticity 104, 369-384 (2011).

63. Svanadze, M. M.: Boundary value problems of the theory of viscoelasticity for Kelvin-Voigt materials, Proc. Appl. Math. Mech. 10, 307-308 (2010).

64. Svanadze, M. M.: Boundary value problems of steady vibrations in the theory of thermoviscoelasticity for Kelvin-Voigt materials, Proc. 9th Int. Congress on Thermal stresses, TS2011, 5-9 June, 2011, Budapest, Hungary, CD of Papers and http ://ts2011.mm.bme.hu/kivonatok/Maia\%20M.\%20Svanadze _TS2011_1294776902.pdf.

65. Svanadze, M. M.: Potential method in the linear theories of viscoelasticity and thermoviscoelasticity for Kelvin-Voigt materials, Technische Mechanik 32, 554-563 (2012).

66. Passarella, F., Tibullo, V., Zampoli, V.: On microstretch thermoviscoelastic composite materials, Europ. J. Mechanics, A/Solids 37, 294-303 (2013).

67. Sharma, K., Kumar, P.: Propagation of plane waves and fundamental solution in thermoviscoelastic medium with voids, J. Thermal Stresses 36, 94-111 (2013).

68. Tomar, S. K., Bhagwan, J., Steeb, H.: Time harmonic waves in a thermoviscoelastic material with voids, J. Vibr. Control (2013), DOI: $10.1177 / 1077546312470479$.

69. de Boer, R.: Theory of Porous Media: Highlights in the historical development and current state. Springer, Berlin (2000), 618 p. 
70. Ieşan, D.: Thermoelastic Models of Continua. Kluwer, Boston (2004), 298 p.

71. Straughan, B.: Stability and wave motion in porous media, Springer, New York (2008), 437 p.

72. Straughan, B.: Heat Waves, Springer, New York, Dordrecht, Heidelberg, London (2011), 318 p.

73. Kellogg, O. D.: Foundations of Potential Theory, Berlin, Springer (1929), $384 \mathrm{p}$.

74. Günther, N. M.: Potential Theory and its Applications to Basic Problems of Mathematical Physics, New York, Ungar Publ Co (1967), 338 p.

75. Hsiao, G. C., Wendland, W. L.: Boundary Integral Equations, SpringerVerlag, Berlin, Heidelberg (2008), 618 p.

76. Cheng, A. H. D., Cheng, D. T.: Heritage and early history of the boundary element method, Engng. Anal. Boundary Elem. 29, 268-302 (2005).

77. Fredholm, E. I.: Sur une classe d'equations fonctionnelles, Acta Mathematica 27, 365-390 (1903).

78. Muskhelishvili, N. I.: Singular Integral Equations, Noordhoff, Groningen, Holland (1958), $447 \mathrm{p}$.

79. Muskhelishvili, N. I.: Some Basic Problems of the Mathematical Theory of Elasticity, Springer Science+Business Media, Dordrecht, (1977), 732 p.

80. Vekua, I. N.: Shell Theory: General Methods of Construction, Pitman Advanced Publishing Program, Boston-London-Melbourne (1985), 287 p.

81. Mikhlin, S. G.: Multidimensional Singular Integrals and Integral Equations. Pergamon Press, Oxford (1965), 259 p.

82. Kupradze, V. D.: Potential Methods in the Theory of Elasticity. Israel Program Sci. Trans., Jerusalem (1965), 340p.

83. Kupradze, V. D., Gegelia, T. G., Basheleishvili, M. O., Burchuladze, T. V.: Three-Dimensional Problems of the Mathematical Theory of Elasticity and Thermoelasticity, North-Holland Publ. Comp., Amsterdam, New York, Oxford (1979), 929 p.

84. Burchuladze, T. V., Gegelia, T. G.: The Development of the Potential Methods in the Elasticity Theory. Metsniereba, Tbilisi (1985), 228 p. (Russian).

85. Gegelia, T., Jentsch, L.: Potential methods in continuum mechanics. Geor- 
gian Math. J. 1, 599-640 (1994).

86. Achenbach, J. D.: Wave Propagation in Elastic Solids. North-Holland Publ. Comp., Amsterdam, Oxford, American Elsevier Publ. Comp., Inc., New York (1975), $425 \mathrm{p}$.

87. Hörmander, L.: The Analysis of Linear Partial Differential Operators II: differential operators with constant coefficients. Springer-Verlag: Berlin, Heidelberg, New York, Tokyo, 1983.

88. Sommerfeld, A.: Die Grensche Funktion der Schwingungsgleichung. Jber. Deutsch. Math. Verein. 21, 309-353 (1912).

89. Kupradze, V. D.: Sommerfeld principle of radiation. Reports Acad. Sci. USSR 2, 1-7 (1934) (Russian).

90. Vekua, I. N.: On metaharmonic functions. Proc. Tbilisi Math. Inst. Acad. Sci. Georgian SSR 12, 105-174 (1943) (Russian).

91. Gurtin, M. E.: The linear theory of elasticity. In: Truesdell, C. (ed): Handbuch der Physik, vol VIa/2, Springer-Verlag, Berlin, Heidelberg, New York, 1-296 (1972).

92. Hetnarski, R. B., Ignaczak, J.: Mathematical Theory of Elasticity, Taylor \& Francis, New York, London (2004), 821 p.

93. Nowacki, W.: Thermoelasticity, Pergamon Press, Oxford (2004).

94. Sandru, N.: On some problems of the linear theory of asymmetric elasticity, Int. J. Engng. Sci. 4, 81-96 (1966).

95. Dragos, L.: Fundamental solutions in micropolar elasticity, Int. J. Engng. Sci. 22, 265-275 (1984).

96. Wang, M. Z., Xu, B. X., Gao, C. F.: Recent general solutions in linear elasticity and their applications, Appl. Mech. Rev. 61, 030803-1-20 (2008).

97. Galerkin, B.: Contribution à la solution générale du problème de la théorie de l'élasticité dans le cas de trois dimensions, C. R. Acad. Sci. Paris 190, 1047-1048 (1930).

98. Iacovache, M.: O extindere a metodei lui Galerkin pentru sistemul ecuatiilor elasticitătii, Bul. St. Acad. Rep. Pop. Române, Ser. A 1, 593-596 (1949).

99. Chandrasekharaiah, D. S.: Complete solutions in the theory of elastic materials with voids -I, Quart. J. Mech. Appl. Math. 40, 401-414 (1987).

100. Chandrasekharaiah, D. S.: Complete solutions in the theory of elastic ma- 
terials with voids -II, Quart. J. Mech. Appl. Math. 42, 41-54 (1989).

101. Knops, R. J., Payne, L. E.: Uniqueness Theorems in Linear Elasticity, Springer, Berlin, Heidelberg, New York (1971).

102. Pompei, A., Scalia, A.: On the steady vibrations of elastic materials with voids, J. Elasticity 36, 1-26 (1994).

103. Pamplona, P. X., Muñoz-Rivera, J. E., Quintanilla, R.: On uniqueness and analyticity in thermoviscoelastic solids with voids, J. Appl. Anal. Comput. 1, 251-266 (2011).

104. Pamplona, P. X., Muñoz-Rivera, J. E., Quintanilla, R.: Analyticity in porous-thermoelasticity with microtemperatures, J. Math. Anal. Appl. 394, 645-655 (2012).

105. Ieşan, D., Quintanilla, R.: On a strain gradient theory of thermoviscoelasticity, Mech. Res. Commun. 48, 52-58 (2013). 